\title{
Dynamical Study of Guest-Host Orientational Interaction in Liquid Crystalline Materials
}

\author{
by \\ Thai Viet Truong \\ B.A. (University of California, Berkeley) 1998 \\ M.A. (University of California, Berkeley) 2001 \\ A dissertation submitted in partial satisfaction of the \\ requirements for the degree of \\ Doctor of Philosophy \\ in \\ Physics \\ in the \\ GRADUATE DIVISION \\ of the \\ UNIVERSITY OF CALIFORNIA, BERKELEY \\ Committee in charge: \\ Professor Yuen-Ron Shen, Chair \\ Professor Herbert L. Strauss \\ Professor Peter Y. Yu
}

Fall 2005 
The dissertation of Thai Viet Truong is approved:
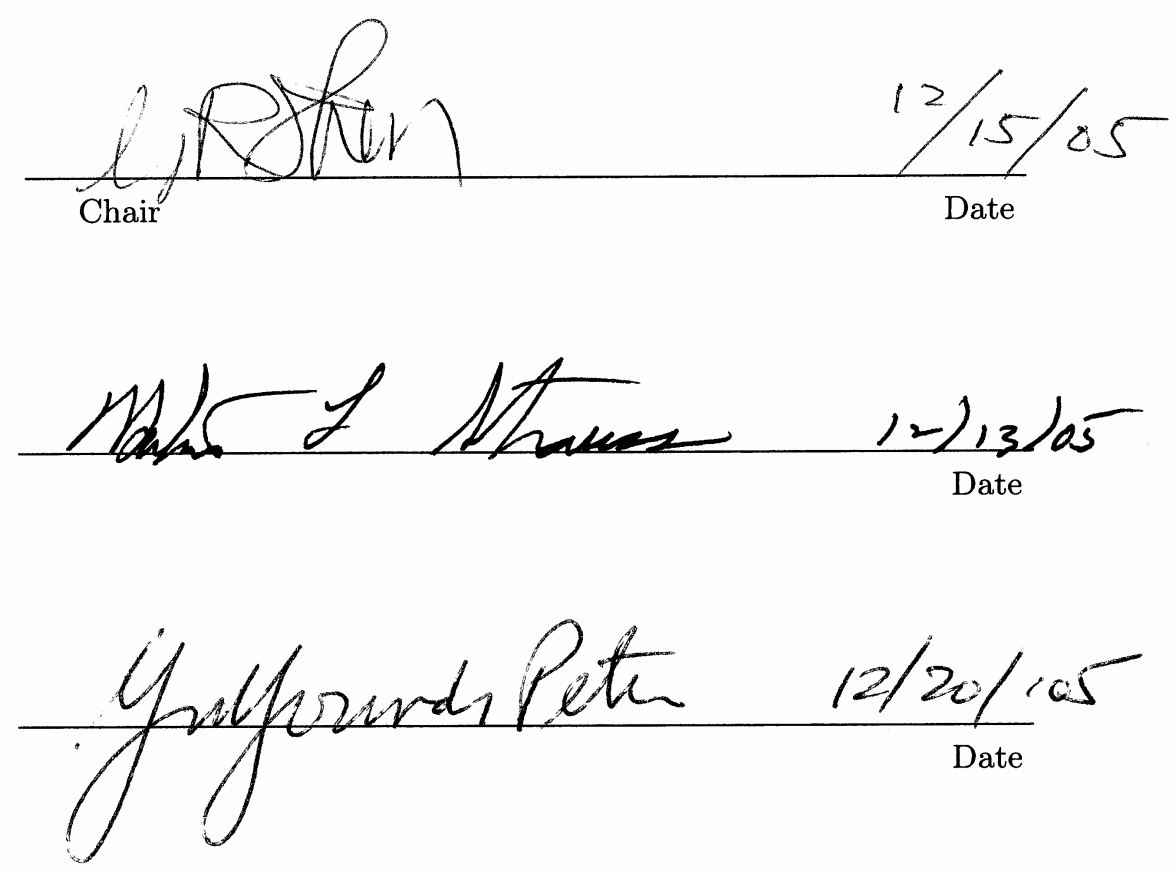

University of California, Berkeley

Fall 2005 


\section{Dynamical Study of Guest-Host Orientational Interaction in Liquid}

Crystalline Materials

Copyright 2005

by

Thai Viet Truong 


\begin{abstract} Materials

by

Thai Viet Truong

Doctor of Philosophy in Physics

University of California, Berkeley

Professor Yuen-Ron Shen, Chair
\end{abstract}

Dynamical Study of Guest-Host Orientational Interaction in Liquid Crystalline

Guest-host interaction has long been a subject of interest in many disciplines. Emphasis is often on how a small amount of guest substance could significantly affect the properties of a host material. This thesis describe our work in studying a guest-host effect where dye-doping of liquid crystalline materials greatly enhances the optical Kerr nonlinearity of the material. The dye molecules, upon excitation and via intermolecular interaction, provides an extra torque to reorient the host molecules, leading to the enhanced optical Kerr nonlinearity.

We carried out a comprehensive study on the dynamics of the photoexcited dyedoped liquid crystalline medium. Using various experimental techniques, we separately characterized the dynamical responses of the relevant molecular species present in the 
medium following photo-excitation, and thus were able to follow the transient process in which photo-excitation of the dye molecules exert through guest-host interaction a net torque on the host LC material, leading to the observed enhanced molecular reorientation. We also observed for the first time the enhanced reorientation in a pure liquid crystal system, where the guest population is created through photoexcitation of the host molecules themselves. Experimental results agree quantitatively with the time-dependent theory based on a mean-field model of the guest-host interaction.

Professor Yuen-Ron Shen Dissertation Committee Chair 
To my parents 


\section{Contents}

List of Figures $\quad$ iv

List of Tables $\quad$ vii

1 Introduction 1

2 Materials $\quad 7$

2.1 Host: Liquid Crystals . . . . . . . . . . . . . . . . . 7

2.1.1 Molecular Structure . . . . . . . . . . . . . . . . . . 7

2.1.2 Liquid Crystal Phases . . . . . . . . . . . . . . . . 8

2.1.3 Alignment and Reorientation of Liquid Crystals . . . . . . . . . . . 11

2.2 Guest: Dyes . . . . . . . . . . . . . . . . . . . . 12

2.3 Guest-host system . . . . . . . . . . . . . . . . . . 15

3 Theory of rotational dynamics of guest-host systems 18

3.1 Physical Model . . . . . . . . . . . . . . . . . . . . . 18

3.2 Formalism of Dynamic Equations . . . . . . . . . . . . . . . . 21

3.2 .1 Dye response . . . . . . . . . . . . . . . . 23

3.2 .2 Pure LC response . . . . . . . . . . . . . . . . . . 25

3.2 .3 Dye-doped LC response . . . . . . . . . . . . . . . . . . . 29

3.3 Transient and steady-state enhancement . . . . . . . . . . . . . . . . 31

3.4 Overview of experimental strategy . . . . . . . . . . . . . . . 34

4 Quasi-steady-state reorientation of dye-doped liquid crystals $\quad 37$

4.1 Experimental setup . . . . . . . . . . . . . . . . 37

4.2 Result and discussion . . . . . . . . . . . . . . . . . . . 39

5 Dynamic response of dye molecules $\quad 42$

5.1 Population decay: transient fluorescence . . . . . . . . . . . . . . 42

5.2 Rotational diffusion: transient dichroism . . . . . . . . . . . . . . 45 
6 Reorientational dynamics of dye-doped liquid crystals $\quad \mathbf{5 4}$

6.1 Experimental setup . . . . . . . . . . . . . . . . . . 54

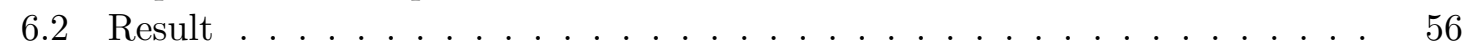

7 Discussion of the reorientational dynamics of dye-doped liquid crystals 66

8 Excitation-enhanced optical reorientation in pure liquid crystals $\quad 72$

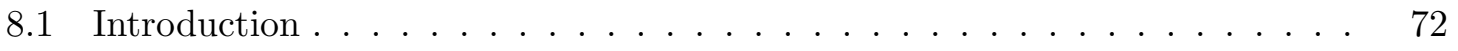

8.2 Theory . . . . . . . . . . . . . . . . . . . 73

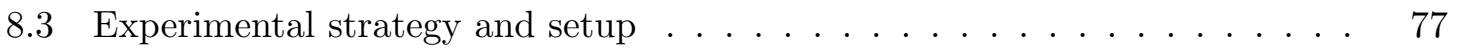

8.4 Result and Discussion ....................... 80

$\begin{array}{lr}\text { Bibliography } & 86\end{array}$

$\begin{array}{ll}\text { A Mean-field description of rotational dynamics } & 94\end{array}$

A.1 Introduction . . . . . . . . . . . . . . . . . . . 94

A.2 Dynamics of dye-doped liquid crystals . . . . . . . . . . . . . . . . . 95

A.3 Dynamics of pure liquid crystals . . . . . . . . . . . . . 103 


\section{List of Figures}

2.1 Schematic of the molecular structure of a typical liquid crystal. . . . . . . .

2.2 Some phases of LC's. The molecules are depicted as cylindrically symmetric rods. The arrows depict the alignment director. . . . . . . . . . . . . . .

2.3 Reorientation of a LC molecule by external electric field $\vec{E}$. Due to anisotropic shape of the LC molecule, the induced polarization $\vec{P}$ is not parallel to $\vec{E}$, leading to a torque that reorients the molecule parallel to the external field.

2.4 Molecular structures of anthraquinone dyes. The dyes consist of the anthraquinone core with electron-donating substituent groups. . . . . . . . . .

2.5 Photoinduced molecular changes. (a) In azo dyes, excitation leads to trans-cis isomerization. (b) In anthraquinone dyes, represented by 1AAQ, excitation leads to intra-molecular charge transfer, with electronic charge transferred from the substituent group the anthraquinone core. Shown is the electron cloud distribution in the highest occupied molecular orbital (HOMO) and the lowest unoccupied molecular orbital (LUMO), following Ref. [35]. Black and gray circles indicate the non-vanishing positive and negative wave-function amplitude, respectively. . . . . . . . . . . . . . . . .

2.6 Absorption and fluorescence spectra of $1 \mathrm{AAQ}$ in 5CB. 5CB absorption has been subtracted out. . . . . . . . . . . . . . . .

3.1 Schematic setup of a pump-probe experiment. . . . . . . . . . .

4.1 Top-view schematic setup of the quasi-steady-state experiment. Pr: probe beam, P: pump beam, S: sample; PD: photodetector. All beams lie on the $\hat{x}-\hat{z}$ plane, with $\mathrm{P}$ polarized along $\hat{y}$. Input polarization of $\operatorname{Pr}$ is set to be along $\hat{x}+\hat{y}$, and analyzer $\mathrm{A}$ is set at the crossed direction $-\hat{x}+\hat{y}$. Signal is from Pr leaking through analyzer A as the pump beams induce optical anisotropy in the sample S. . . . . . . . . . . . . .

4.2 Optical Kerr signal versus pump intensity, from dye-doped and pure LC samples. Solid curves are quadratic fits. . . . . . . . . . . 
4.3 Steady-state enhancement factor versus pump wavelength. Solid line is the scaled absorption spectrum. . . . . . . . . . . . . .

5.1 Schematic setup of transient fluorescence experiment. S: dye-doped LC sample, I: iris, Pol-1 and Pol-2: polarizers, K: Kerr cell filled with carbon disulfide, PD: photodetector. . . . . . . . . . . . . . . . . .

5.2 Fluorescence intensity, polarized at the magic angle with respect to the pump beam polarization versus time. Solid line is single exponential fit. . . . . . .

5.3 Top-view schematic of the experimental setups. Pr: probe beam; P1, P2: pump beams \#1 and \#2; S: sample; PD: photodetector. All beams lie on the $\hat{x}-\hat{z}$ plane, with $\mathrm{P} 1$ and P2 polarized as shown. Input polarization of $\operatorname{Pr}$ is set to be along $\hat{x}+\hat{y}$, and analyzer $\mathrm{A}$ is set at or near $-\hat{x}+\hat{y}$. Signal is from Pr leaking through analyzer A as the pump beams induce optical anisotropy in the sample S. . . . . . . . . . . . . .

5.4 Schematic demonstration of the two-pump/probe technique to measure ground state rotational dynamics. Wavelength of pump pulses 1 and 2 is in resonance with the $S_{o}-S_{1}$ transition. Wavelength of probe pulse is varied to measure state-specific rotational dynamics. Pulse polarization shown is along the $x-y$ plane. See text for explanations. . . . . . . . . . . . . . . .

5.5 Dye excited-state dichroism probed at $600 \mathrm{~nm}$. (a) $S_{t}(\gamma, t)$ versus $\gamma$ at $t=340$ ps, induced by pump pulse P1 alone (at $t=0$ ) and by pump pulse P1 followed by pump pulse $\mathrm{P} 2$ (at $t=320 \mathrm{ps}$ ) that reduces the excited state dichroism to zero. The dichroism is determined from the abscissa of the fitted parabolic minimum, which appears at $\gamma=0$ in the absence of the pump pulse (inset). (b) Transient dichroism signal $S(t)$ measured by taking the difference between $S_{t}(-\gamma, t)$ and $S_{t}(+\gamma, t)$. (c) Single exponential fit of the same $S(t)$ shown in (b). Inset shows how the dichroism induced by $\mathrm{P} 1$ is reduced to zero by P2.

5.6 Transient dichroism probed at $505 \mathrm{~nm}$, induced by pump pulses $\mathrm{P} 1$ at $t=0$ ps and P2 at $t=320$ ps. After P2, the observed dichroism reverses sign and decays exponentially. Inset shows the single-exponential fit of the experimental data after $\mathrm{P} 2 \ldots \ldots \ldots \ldots \ldots \ldots$

6.1 Top-view schematic of the transient grating setup. Pr: probe beam; P1, P2: pump beams \#1 and \#2; S: sample; PD: photodetector. All beams lie on the $\hat{x}-\hat{z}$ plane, with polarizations as shown. P1 and P2 overlap spatially and temporally at sample $\mathrm{S}$, forming a polarization grating which diffracts $s$-polarized $\operatorname{Pr}$ as the $p$-polarized signal at the Bragg angle. . . . . . . 55

6.2 Result of the transient grating experiment from (a) pure LC at probe-wavelength of $848 \mathrm{~nm}$ and from dye-doped LC at (b) $848 \mathrm{~nm}$, (c) $925 \mathrm{~nm}$, and (d) 1024 nm. Solid lines are theoretical fits. The signal from dye-doped LC are fitted with $\eta_{\infty}=3.3$ and $r_{u}=0.6$. See text for fitting procedure. . . . . . . . . 
6.3 The four components of $\Delta \alpha_{h}^{\prime} Q_{h}^{\text {pure }}(t)$, described by Eq. (3.20), used to obtain the fit in Fig. 6.2a for the transient grating signal from pure LC. Vertical scale is the same as in Fig. 6.2. . . . . . . . . . . . . . .

6.4 Fitting of the dye torque: (a) Excited and ground state dye anisotropies. Note the negative sign on $Q_{g}(t)$. (b) Net transient torque from the dye population acting on the LC host, represented by $Q_{e}(t)+r_{u} Q_{g}(t)$, for different values of $r_{u}$. (c) LC anisotropy created by the dye torque, $Q_{h}^{e n h}(t)$, for different values of $\eta_{\infty}$ and $r_{u}$. Vertical scale of (c) is the same as in Fig. 6.2.

6.5 Fitting of the transient grating signal from dye-doped LC: (a) The five terms (Eq. (32)) used to obtain the fit in Fig. $6.2 \mathrm{~b}$, where $\eta_{\infty}=3.3$ and $r_{u}=$ 0.6. Note the negative sign on $\Delta \alpha_{e}^{\prime} Q_{e}$. Inset of (a) gives the corresponding transient enhancement factor $\eta(t)$. (b) For different values of $r_{u}$, with the dye contribution to the signal adjusted as shown, the fit remains practically the same as in Fig. 6.2b. Vertical scale is the same as in Fig. 6.2. . . . . . .

7.1 Interaction between excited $1 \mathrm{AAQ}$ and 5CB that could enhance intermolecular friction upon excitation of 1AAQ. Solid arrows indicate the dipole moments. Dotted line indicates the hydrogen-bond between the molecules. . .

8.1 Schematic setup of the experiment. Pr: probe beam, P: pump beam, S: sample; QWP: removable quarter-wave plate for probe beam; A: analyzer polarizer; PD: photodetector. . . . . . . . . . . . . . .

8.2 Time evolution of the dichroism $(\bullet)$ and birefringence $(\square)$ of the pure liquid crystal sample following photoexcitation at $t=0$. Dotted line is to guide the eye. Solid line in inset is single exponential fit of the dichroism data following Eq. (8.15), yielding $\tau_{Q_{e}}$. Solid line in main graph is bi-exponential fit of the birefringence data following Eq. $(8.16) \ldots \ldots \ldots \ldots$ 


\section{List of Tables}

2.1 Molecular structures of some representative LC's. . . . . . . . . . . . . . 9

6.1 Relative magnitudes of dye polarizabiblity anisotropies from the transient grating experiment, from the fits in Fig. 6.2b-d. $\Delta \alpha_{g}^{\prime}$ at $\lambda_{\text {probe }}=848 \mathrm{~nm}$ is estimated to be $\simeq 2 \AA^{3} \ldots \ldots \ldots \ldots \ldots$ 


\section{Acknowledgments}

First and foremost, I want to express my deepest gratitude to my advisor, Prof. Yuen-Ron Shen, for his guidance and nurturing over the years. I continue to be amazed at the dedication that he brings to his work, and to the training of graduate students like myself. I am forever grateful for the seemingly limitless patience that he has in steering and shaping me into something resembling an independent researcher. I am sure his direct, intuition-filled way of doing science will continue to inspire and guide my career in the future.

I am indebted to Professor Shen not only for having guided and nurtured me directly, but also for having led a truly dynamic and wonderful research group that any graduate student would love to be a part of. I have learned much science and gained much cultural knowledge through my interaction with the very diverse and international group of people that have passed through our research group.

Special thanks go to Lei $\mathrm{Xu}$ for his guidance and camaraderie in the two years that we worked together. I will always remember with fondness the many nights that we worked late together, the early-morning dinners that we shared, and Lei's many words of encouragement when we couldn't understand the experimental results. I got my first handson training in experimental physics with Markus Raschke, whose precise and thorough style of doing things I try to emulate. Good times and fruitful interaction were also shared with (in rough chronological order) Joachim Diener, Boris Averboukh, Rupert Huber, Wolfgang Becker, Kok-wai Cheah, Karl-Heinz Ernst, Chun Zhang, Masahito Ohe, Katsuyoshi Ikeda, 
Francois Lagugne, and Pasquale Pagliusi.

I remember the previous generation of graduate students, Doseok Kim, Paulo Miranda, Xing Wei, and Seok-Choel Hong, with much admiration and gratitude. I just wished that they had left us with a more organized lab! Xing's reply of "You can calculate it!" to many of my questions for him when I first joined the lab continued to inspire me to this day to figure things out for myself.

The current and recent group of students and postdocs that I share the basement with are simply wonderful individuals and have made the (many) years of my graduate career truly enjoyable. I could only hope that my future work environment would resemble what I have experienced in the Shen group. I will always treasure the help and companionship that Song-Hee Han has provided. John McGuire is an example of rigor, both in physics and salsa dancing. From Mikhail Belkin I gained an appreciation for the direct, brute force experimental approach (such as fixing the malfunctioning laser power supply by hitting it sharply with your knuckles). Victor Ostroverkhov is the go-to guy in the lab, always ready to share an insight in electronics or to help find a piece of optics. Ji $\mathrm{Na}$ is the most congruent anthropologist-physicist-chemist combination that one can hope to find. I am indebted to Victor and Jina for much technical help using Latex in preparing this thesis. Luning is another chemist in our group who, together with Ji Na, have expanded my scientific education by bringing in the chemist's perspective. Weitao Liu is the newly crowned, and always generous, queen of hidden lab treasures. Gene-wei Li's gentle manners and sharp mind make a perfect office-mate. Eric Chen fills the basement with his hearty laughs, and I benefit much from his practical knowledge of liquid crystals. I wish the best 
of luck to the two newest additions to the group, Feng Wang and Evgenia Kim.

Thanks to my friends outside of the research group for the great times that we have shared and the support that they have provided. I have learned much about life from them.

I would like to thank Lien Tran for the companionship throughout a big part of my academic life. I will always treasure the times that we have shared together.

This work would not have been completed without the constant love and support of Hanh Nguyen. She has filled my life with joy and happiness, and made me a much better person. She has been my perfect companion through life, and I constantly strive to be deserving of her dedication and love.

Lastly, I have been most fortunately to have a family that have always supported and loved me. They have formed the core of who I am today, and are my constant source of inspiration. My late sister Anh-Thi Truong, my sisters Anh-Tho Truong and Vi-Thanh Truong, and my brothers Thang Truong, Thuan Truong, and Tom Truong are always in my heart. My sister Vi-Thanh's newly formed family, with my brother-in-law Vann Tran and my little nephew Donovan Duy Tran, reminds me that the cycle of life is turning, and urges me to step up and take the new role of uncle seriously. And my mom and dad, they have loved, taught, and nurtured me, providing me with an example of a forthright way of life, and allowing me to find my own way in life. I will never be able to repay all the hardships and sacrifices that they have gone through for me. I dedicate this thesis to my parents. 


\section{Chapter 1}

\section{Introduction}

It has been long appreciated in a wide number of fields how a small amount of guest substance could interact with a host material and drastically change the properties of the host material. This guest-host interaction has been exploited in designing new materials with desirable properties which are not present in the pure host materials. Also, investigations of such guest-host systems often yield insights into the material system that would not be possible from studying the host materials alone.

Liquid crystals (LC) are materials that exhibit strong intermolecular interaction and can exist in states that are intermediate between isotropic liquids and crystalline solids. Due to the correlated nature of LC's, often the effect of impurities is greatly amplified. Guest-host interactions has long been used to both modify the properties and gain understanding of the LC host materials. Examples include impurity-induced changes of phase transition temperatures, optical Kerr nonlinearity, photorefractivity, and modification of chiral structures of LC's $[1,2,3,4]$. 
Beside the already huge role that LC's play in the display industry, in the last decade or so there has been a push to take advantage of the large optical Kerr nonlinearity of LC's in a wide variety of device-applications in the optoelectronics industry $[4,5,6]$. The large optical Kerr nonlinearity of LC's comes from their highly anisotropic molecular structures, making them readily reoriented by an optical field [5, 6]. In 1990, Jánossy discovered that the optical reorientation of LC's can be enhanced up to two orders of magnitude upon doping with certain absorbing dichroic dyes when the dye molecules are excited anistropically [7]. Phenomenologically, the enhanced reorientation could be described by an extra torque that is proportional to the optical torque, though the material parameters that couple with the optical torque of the dye-doped materials remain practically the same as the pure materials. This enhancement of the already large optical nonlinearity of LC's by dye-doping, which has come to be known as the Jánossy effect, has obviously attracted much attention of researchers looking for materials with large nonlinearity for use in the optoelectronics industry. From a scientific standpoint, the Jánossy effect raises interesting questions about the nature of the guest dye-LC host interaction that gives rise to the enhanced molecular reorientation.

Experimental works subsequent to Jánossy's discovery $[8,9,10]$ showed that while the effect relies on optical excitation of the dye, it is not thermal in nature, as different dyes with the same absorption strength yield different enhancement effect. The enhanced reorientation could have either positive or negative sign, i.e. the extra torque resulting from dye excitation could reorient the host molecules either parallel or perpendicular to the linearly polarized light field $[10,11,12]$. Investigation of how the effect depends on 
the molecular structures of the guest and host showed that it is sensitive to the structural change of the substituent groups in the dye molecule, pointing to the importance of dipolar interactions between guest and host molecules $[13,14,15]$. The dyes that cause the effect are known not to undergo photoinduced conformational changes, as in the case with the azo dyes [16]. The enhanced optical reorientation due to dye-doping was also found to be not limited to the liquid crystalline phase, but also present in isotropic liquids, seemingly with the same mechanism at work $[17,18,19]$. Thus the effect is quite general.

Jánossy has proposed a mean-field model to explain the effect [20], which is based on two main processes. First, optical excitation of the dichroic dye yields oriented molecules in the excited-state population and orientational "holes" in the ground-state population. Then, the dye molecules and holes in the excited and the ground states, respectively, exert different reorienting torques on the host molecules through their respective molecular orientational couplings with the host, resulting in a net torque on the host molecules and hence the enhanced reorientation. In Jánossy's model, the guest-host interaction is manifested as a mean-field orientational coupling energy between guest and host molecules. Marrucci et al. $[18,21]$ recognized that in addition to the anisotropic orientational energy coupling, the isotropic interaction between guest and host molecules, which is manifested in the rotational diffusion coefficients of the guest molecules, could also play a role in determining the extra reorienting torque.

The key assumption of the model described above is that upon photoinduced excitation of the guest molecules, the guest-host orientational interaction and the rotational diffusion of the guest change significantly. Until recently, this assumption has not been 
independently tested. The extra torque on host molecules generated by the dye is inherently transient, lasting only when the anisotropic population of the dye excited and ground states are present. Nevertheless, only steady-state or quasi-steady-state behavior of the Jánossy effect was investigated in the earlier studies. As is well known, comparing model predictions with experimental observations in the transient regime would certainly constitute a more stringent test of the model. Thus, though the model described above has been consistent with the results found in earlier experimental studies (for a review see $[22,23,24]$ ), it cannot be said that experiments have tested successfully the validity of the model.

This thesis describes our effort to study the Jánossy effect in the dynamical regime, in the isotropic phase of the $\mathrm{LC}[25,26,27]$. We set out to measure the orientational dynamics of both guest and host molecules, and thus be able to follow the transient reorienting torque that the guest exerts on the host. We would like to learn from the early dynamics of the system whether the mean-field model for the guest-host interactions is still valid during the period when various molecular relaxations just begin to set in. Our work would not only provide a thorough test of the validity of the model described above, but also provide further insights into the guest-host interactions responsible for the enhanced optical reorientation.

In Chapter 2 we describe briefly the qualitative properties of the LC host and the dye guests as relevant to our study. Chapter 3 describes the physical model and theoretical formalism that we used in describing the guest-host system. It also describes our general experimental strategy, where we used various techniques to carry out a series of quasisteady-state and time-resolved measurements of the optical Kerr responses of both pure 
and dye-doped LC systems to characterize separately the picosecond to nanosecond orientational dynamics of host molecules, excited-state guest molecules, and ground-state guest molecules. This allow us to follow the host response to the reorienting torque generated by the excitation of the guest molecules. Chapters 4,5 , and 6 describe in details the three sets of experiments that were carried out to investigate, respectively, the quasi-steady-state response of the guest-host system, the transient response of the dye guest, and the transient response of the dye-doped LC. The mean-field model of the guest-host interaction described above was found to agree well with our experimental observation. We discuss the relevance of our findings in Chapter 7.

An important prediction of the model is that the Jánossy effect should be present in pure LC system, provided that the optical field anisotropically excites the LC molecules themselves. The excited LC molecules, and the orientational holes that they leave behind in the ground-state population, then serve as the guest population, qualitatively much like the dye molecules, to provide the extra torque in reorienting the ground-state LC molecules. We set out to experimentally test this prediction and describe our preliminary result in Chapter 8. We indeed found the presence of the Jánossy effect in a pure LC system. This further confirms the validity of the model and provides a new and simple way to enhanced the optical nonlinearity of LC materials.

The Jánossy effect could be seen as a special case of the solvation process, a subject that is of wide interest. (For a review of solvation, see Refs. [28, 29, 30] and references therein.) In the past, solvation was often studied through observation on reporter chromophores that reflect the solvent effects on the reporter molecule. Only recently have 
there been studies that looked at the response of the solvent, which provides complementary information on solvation $[31,32,33]$. Seen in this light, our study can be considered a comprehensive study of the orientational solvation dynamics of a guest-host system. 


\section{Chapter 2}

\section{Materials}

\subsection{Host: Liquid Crystals}

Liquid crystals, as the name implies, are materials that could exist in phases intermediate between the isotropic liquid and solid crystalline phases. These LC phases, also called mesophases, possess macroscopic long-range order in at least one degree of freedom. There is no lattice, hence the materials remain relatively fluid. Various external parameters determine which phase the material will be in. An important class of LC, called the thermotropics, exhibit various LC phases as a function of temperature. Our work deals exclusively with this class of LC's.

\subsubsection{Molecular Structure}

LC's derive their unique properties from their highly anisotropic molecular structures. We focus our attention on the LC's that have elongated structures, as shown in Fig. 


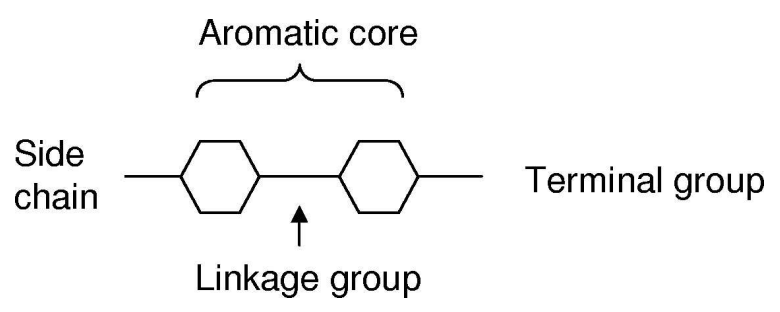

Figure 2.1: Schematic of the molecular structure of a typical liquid crystal.

2.1. The basic structure of such a LC molecule consist of an aromatic core, with a side group and a terminal group at the two ends. Shown in Table 2.1 are molecular structures of several representative LC's. Because of their elongated structures, for most applications these molecules are approximated well as rigid rods with centrosymmetric symmetry. If individual molecules carry a permanent electric dipole, they will assemble in such a way (e.g. head-to-toe) so that the bulk dipole moment is vanishing.

\subsubsection{Liquid Crystal Phases}

The mesophases come about because of the intermolecular interaction between neighboring LC molecules. For the LC materials presented in Table 2.1, intermolecular interactions give rise to characteristically strong orientational correlation between adjacent molecules. The intermolecular interaction consists of the familar modes of interaction: steric, van der Waals, and dipole-dipole, with one or more modes being dominant depending on which specific molecular structure is involved [1,34].

Fig. 2.2 depicts several important phases that LC's can exist in. Lowering the temperature of the material from the isotropic phase one encounters the nematic phase, 
- 4-pentyl-4'-cyanobiphenyl (5CB)

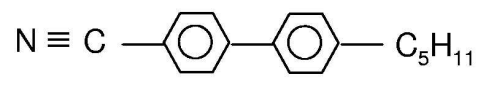

- 4-methoxybenzulidene-4'-butylaniline (MBBA)

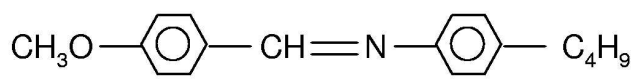

- 4-octyl-4'-cyanobiphenyl (8CB)

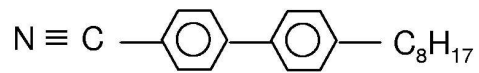

Table 2.1: Molecular structures of some representative LC's.

where there is long-range orientational ordering of the long axes but no translational ordering of the center-of-masses of the molecules. In this phase the molecules are free to move around, but their orientation is restricted along a particular axis, called the director. If the constituent LC molecules are chiral, then the material can form the chiral nematic phase, also known historically as the cholesteric phase. In this phase, locally the molecules follow nematic ordering, but on a larger scale the director itself traces out a helical twist with spatial periods ranging from sub microns to tens of microns.

Because of the characteristically strong orientational coupling between the LC molecules, there exists short-range ordering even in the isotropic phase, as long as the temperature is not too high above the nematic-isotropic transition temperature. (This is not shown in Fig. 2.2.) This short-range ordering is characterized by a correlation length $\xi$ given by [34]:

$$
\xi(T)=\xi_{o}\left[T^{*} /\left(T-T^{*}\right)\right]^{1 / 2}
$$



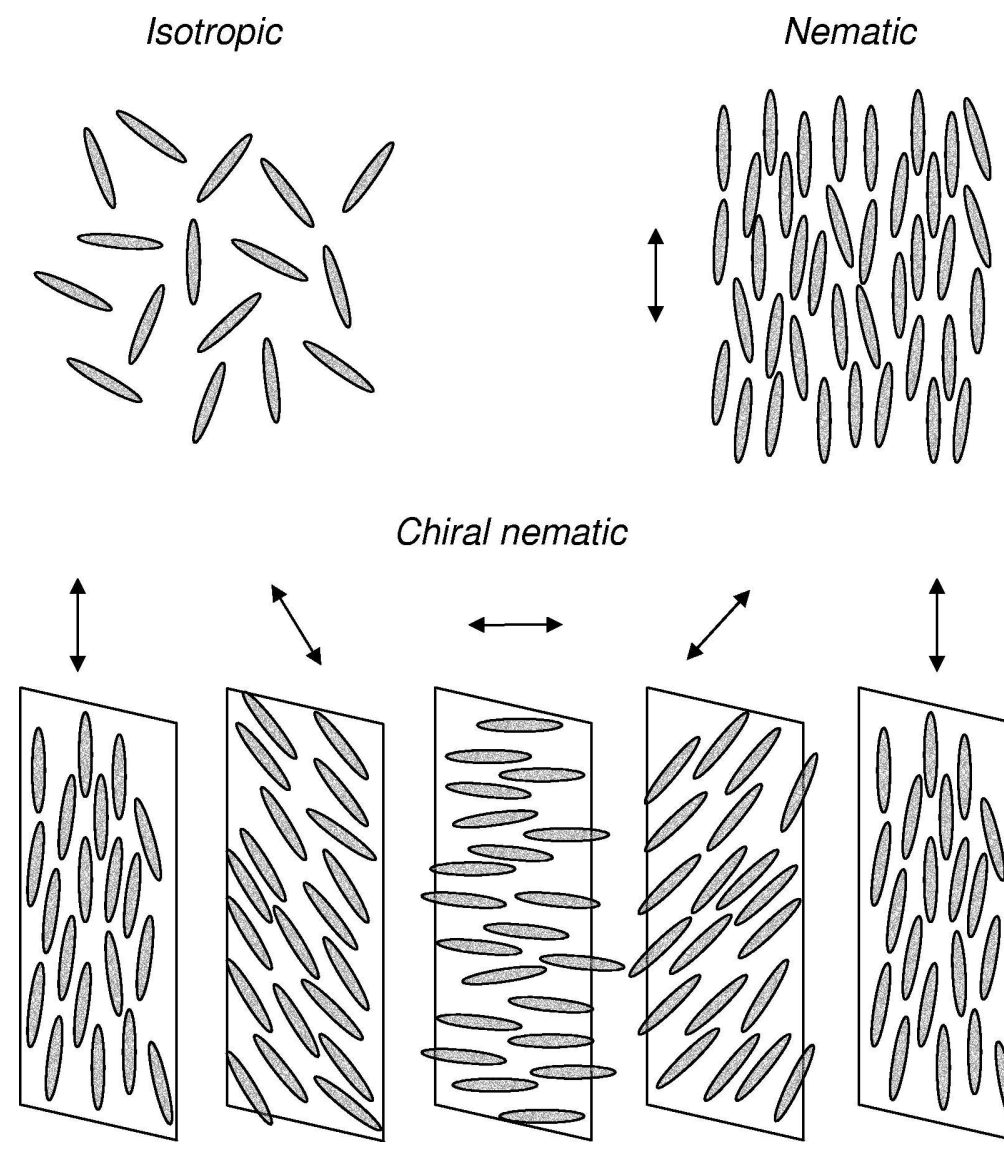

Chiral nematic
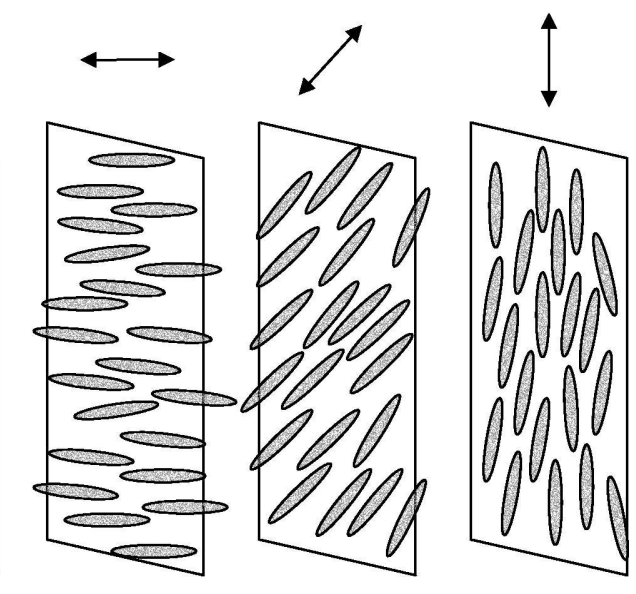

Figure 2.2: Some phases of LC's. The molecules are depicted as cylindrically symmetric rods. The arrows depict the alignment director.

where $\xi_{o}$ corresponds to the LC molecular length and $T^{*}$ is approximately the transition temperature (see section 3.3.2 for a rigorous definition of $T^{*}$ ). For $5 \mathrm{CB}, \xi_{o} \simeq 0.5 \mathrm{~nm}$ and $T^{*} \simeq 35^{\circ} \mathrm{C}$, so we see that for $T-T^{*} \approx 1^{\circ} \mathrm{C}$, we have $\xi \simeq 10 \mathrm{~nm}$. This short-range ordering in the isotropic phase of LC is usually visualized as pseudo-nematic domains of size $\xi$ where molecules within the domain have a net averaged direction but the domains' directors are randomly oriented, giving rise to a macroscopically isotropic system. 


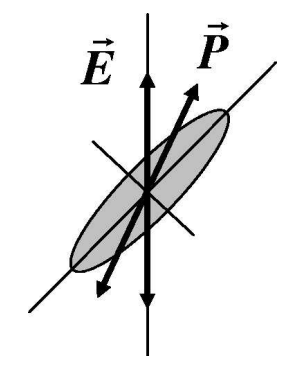

Figure 2.3: Reorientation of a LC molecule by external electric field $\vec{E}$. Due to anisotropic shape of the LC molecule, the induced polarization $\vec{P}$ is not parallel to $\vec{E}$, leading to a torque that reorients the molecule parallel to the external field.

\subsubsection{Alignment and Reorientation of Liquid Crystals}

Practical applications of LC's rely on achieving and controlling alignment of the LC's over extended distances, typically in the range of microns and above. Static alignment is usually achieved through substrate-mediated anchoring, while dynamic controlling of alignment is achieved through the use of external fields. Fig. 2.3 shows schematically how an electric field could reorient a LC molecule. Because of the anisotropy in the LC molecular structure, the polarizabilities along the long and short axes will be different, hence the induced polarization $\vec{P}$ is not parallel to the external field $\vec{E}$. As a result, a torque develops between $\vec{P}$ and $\vec{E}$, reorienting the molecule so that its long axis becomes parallel to the field. (A magnetic field could similarly reorient LC's.)

The electric field in Fig. 2.3 could come from an optical wave, in which case we have optical reorientation of the LC's. As the optical wave travels through the LC, optical reorientation changes the optical property of the material itself, which in turn affects the 
propagating optical wave, leading to the observed large optical nonlinearity of LC materials. This is the optical Kerr effect, and the associated nonlinearity is known as the optical Kerr nonlinearity. In the Jánossy effect, dye-doping modifies this optical reorientation. The torque interaction between the optical field and the LC molecules depicted in Fig. 2.3 is unchanged in a dye-doped LC system. Rather, the dye population, resonantly excited by the optical field, provides an extra torque that acts, via intermolecular interaction, on the LC molecules. Before we describe in details this extra reorienting torque, let us describe the molecular properties of the dyes that produce the Jánossy effect.

\subsection{Guest: Dyes}

The Jánossy effect was discovered when LC's were doped with a particular family of dyes called the anthraquinones. As shown in Fig. 2.4, these dyes share the anthraquinone core, with different electron-donating substituent groups located at various positions about the core. These dyes have absorption bands that are generally either parallel or perpendicular to the long molecular axis (defined as the axis of the 3-ring backbone), with the lowest absorption band in the visible range [35].

Doping of liquid crystals with dyes has a long history. LC's, mainly by design, are transparent in the near-infrared and visible region. Hence when one desires the LC's to have optical absorption, dye-doping is used. Optical absorption of guest dyes in LC's could lead to a variety of consequences, such as thermal loading or creation of new molecular species due to photochemical transformation, which then modify the properties of the LC 
<smiles>O=C1C(=O)C2C=CC=CC2C1=O</smiles>

Anthraquinone

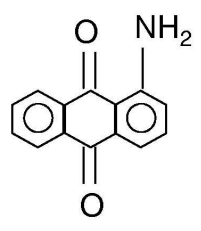

1-Aminoanthraquinone (1AAQ)

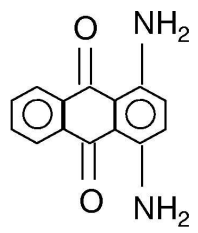

1,2- Diaminoanthraquinone $(2 A A Q)$

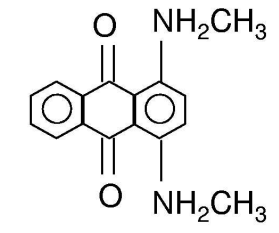

Methyl-1,4-diaminoanthraquinone (M2AAQ)

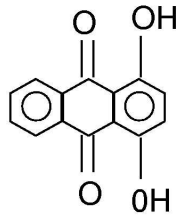

(4-Methylphenyl)-1,4-diaminoanthraquinone (MP2AAQ)

Figure 2.4: Molecular structures of anthraquinone dyes. The dyes consist of the anthraquinone core with electron-donating substituent groups.

host.

There is a family of dyes, the azo dyes, that are known to undergo trans-cis isomerization upon photoexcitation (Fig. 2.5a). This large conformational change of molecular shape due to photoexcitation of the azo dyes have been shown capable of reorienting not only liquid crystals, but also polymers and thin solid molecular films [16, 36]. Currently there is much effort to incorporate the azo dyes in such applications as optical storage and material optical processing.

The anthraquinone dyes that exhibit the Jánossy effect, unlike the azo dyes, are known to not undergo isomerization, nor any other large conformational changes, upon photoexcitation. Also, unlike the azo dyes that almost always provides a torque that reorients the host opposite to the optical torque, many anthraquinone dyes yield positive 
(a) Azo dye

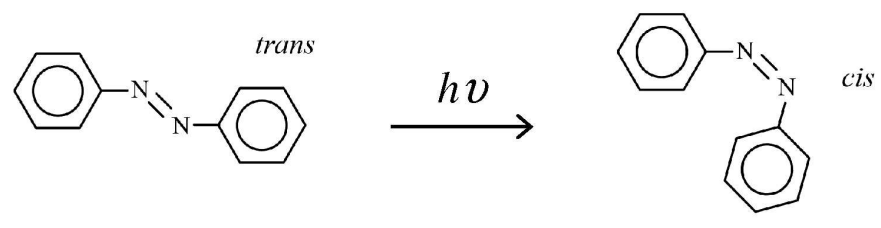

(b) Anthraquinone dye $1 \mathrm{AAQ}$

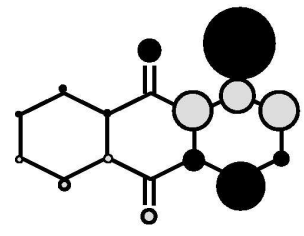

HOMO

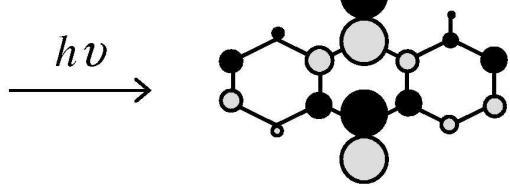

LUMO

Figure 2.5: Photoinduced molecular changes. (a) In azo dyes, excitation leads to transcis isomerization. (b) In anthraquinone dyes, represented by $1 \mathrm{AAQ}$, excitation leads to intra-molecular charge transfer, with electronic charge transferred from the substituent group the anthraquinone core. Shown is the electron cloud distribution in the highest occupied molecular orbital (HOMO) and the lowest unoccupied molecular orbital (LUMO), following Ref. [35]. Black and gray circles indicate the non-vanishing positive and negative wave-function amplitude, respectively.

enhancement of the optical torque.

The anthraquinone dyes are known to undergo a more subtle molecular change upon excitation to the first singlet excited state: the transfer of electronic charge from the substituent group(s) to the anthraquinone core, particularly the carbonyl groups [35], as shown in Fig. 2.5b for the representative molecule 1AAQ. The charge transfer leads to significant difference in electrostatic properties between the ground and excited states, 
e.g. the excited state would be dipolar, and in the case of $1 \mathrm{AAQ}$, the H's of the amino group would be more prone to form hydrogen bonds. This could lead to an overall stronger intermolecular interaction between the dye and a polar/hydrogen-bonding host molecule when the dye is excited, thereby facilitate the Jánossy effect as proposed by the model presented in the previous chapter. This qualitative description of the molecular-structure dependence of the effect has been generally verified by several studies that investigated the effect in a homologous series of anthraquinone dyes/liquid crystal guest-host systems $[13,14,15]$.

\subsection{Guest-host system}

For our study, we focus on the dye $1 \mathrm{AAQ}$, which has a relatively simple structure among the anthraquinone dyes and is known to have a large positive enhancement effect. The absorption and fluorescence spectra of 1AAQ is shown in Fig. 2.6. The first singlet electronic excited state, centered around $475 \mathrm{~nm}$, has the transition dipole moment a few degrees away from the molecular long axis [35], and is approximated to be parallel to the long axis in our study. The fluorescence spectrum of 1AAQ exhibits the characteristic Stoke shift and extends to about 750nm. Depopulation of the first excited state is mainly through nonradiative decay - radiative decay only accounts for a few percent, and conversion to the triplet state is negligible.

For the host material, we chose the well-studied liquid crystal 5CB (Table 2.1). The molecular structure of this LC consists of a biphenyl core, a 5-carbon alkyl side chain, 


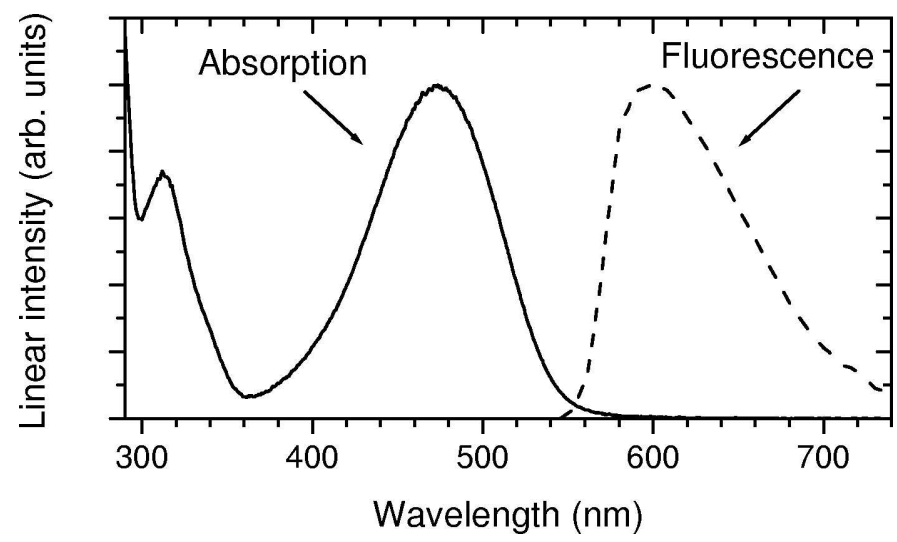

Figure 2.6: Absorption and fluorescence spectra of 1AAQ in 5CB. 5CB absorption has been subtracted out.

and a cyano terminal group that makes the molecule polar. 5CB is optically transparent from the near-IR up to the near-UV range and has the nematic-isotropic transition temperature $T_{n i}=35.3^{\circ} \mathrm{C}$. Our goal is to study the enhanced optical reorientation of dye-doped LC in the transient regime. Specifically, we would like to map out the time evolution of the rotational motion of the LC molecules due to the optical field, with and without dyedoping. For this purpose, the isotropic phase of LC presents a more convenient testing ground than the nematic phase, where the effect was first discovered. In the mesophases the molecular rotational motion necessarily involves complex collective behavior, involving not only molecular properties but also elastic properties of the system as a whole, coupled with surface anchoring. In the isotropic phase, on the other hand, the rotational dynamics involves orientational diffusion which essentially could be described as a single molecule process (though it is slightly complicated by the short-range orientational correlation discussed in section 2.1.2, as we will show in the next section). Thus we chose to study the 
guest-host system in the isotropic phase.

Though the rotational dynamics in the isotropic phase is simpler than in the nematic phase, the nature of the guest-host interaction that leads to the Jánossy effect is essentially the same in the two phases, as have been discussed before $[19,22]$ and will be clear from our forth-coming discussion. Thus the insights gained from our study into the mechanism of the effect should be of direct application to the nematic phase. Furthermore, studying optical reorientation of isotropic LC deserves merit in its own right. Recent studies have shown that isotropic LC may be superior in comparison with mesophased LC for certain applications, due to smaller scattering loss and faster relaxation times. Also, LC in the isotropic phase could serve as a bridge of our understanding of liquid crystalline systems and simple isotropic liquids. Specifically, understanding the reorientation of host LC molecules by dye molecules could shed insights to the solvation process, contributing to a further understanding of the liquid state.

The guest-host system was $0.05 \%$ by mass of $1 \mathrm{AAQ}$ doped into $5 \mathrm{CB}$, in the isotropic phase at $50^{\circ} \mathrm{C}$, unless otherwise noted. The doping concentration yields a number ratio $\sim 1: 2000$ of dye to LC molecules (number density of host and dye are $2.39 \times 10^{21} \mathrm{~cm}^{-3}$ and $1.33 \times 10^{18} \mathrm{~cm}^{-3}$, respectively). The materials were purchased from Aldrich and Merck, and were used without further purification. The samples were held in commercial quartz cells of $1 \mathrm{~mm}$ thickness, and temperature controlled to $\pm 0.1^{\circ} \mathrm{C}$. With the guest-host system described, we next move on to the theoretical description of the guest-host interaction. 


\section{Chapter 3}

\section{Theory of rotational dynamics of}

\section{guest-host systems}

\subsection{Physical Model}

We adopt here the model first proposed by Jánossy [20] and extended by Marrucci et al. $[18,21]$. Consider a guest-host system consisting of the LC host and the dye guest both of which have effective rod-like molecular structures. An incoming linearly polarized optical pump field has two effects on molecules that may directly create an anisotropic orientational distribution of molecules. One is to reorient the molecules toward the field direction by the optical torque against thermal randomization. The other is to excite the molecules to an excited state preferentially along the field direction. The latter process is generally much more effective in creating the anisotropy than the former, and therefore, if present, easily dominates over the former. In our study, the pump field excites only the dye 
molecules and not the LC molecules. Then concerning the direct action of the optical field, we need to consider only the polarized excitation of the dye molecules and the field-induced reorientation of the LC molecules.

The optical excitation of the dye molecules results in three different molecular species in the system: ground-state dye molecules, excited-state dye molecules, and host LC molecules, denoted by the sub-indices $i=g, e$, and $h$, respectively. The excited-state dye molecules have an orientational distribution peaked along the linear polarization of the pump field while the ground-state dye molecules have a corresponding hole in the orientational distribution. Both are influenced by orientational relaxation (due to rotational Brownian motion) and spontaneous de-excitation of the dye molecules. The anisotropic distributions of both the excited-state and the ground-state dye molecules can reorient the LC host molecules via guest-host intermolecular interactions. The former tries to align the LC molecules along the field, and the latter perpendicular to the field. We expect an enhanced reorientation effect on the LC host if the former action is stronger than the latter, but the opposite effect if the reverse is true.

A natural question arises on whether the guest-host interaction can be strong enough to yield significant enhancement of the optical-field-induced reorientation, considering that the concentration of the excited-state dye molecules is quite small. We provide here an order-of-magnitude comparison of the average reorientational potentials exerted on a LC molecule due to the direct optical torque and due to the guest-host interaction. Regarding the former, a light pulse of intensity $I$ produces on a LC molecule a reorientational potential [37]: $V_{\text {direct }} \approx 2 \pi \Delta \alpha^{\prime} L^{2} I / n c$, where $\Delta \alpha^{\prime}$ is the polarizability anisotropy of the LC molecule, 
$L=\left(n^{2}+2\right) / 3$ the Lorenz-Lorentz local-field factor, $n$ the average refractive index, and $c$ the light speed in vacuum. For a typical LC molecule [38], $\Delta \alpha^{\prime}=20 \AA^{3}$ and $n=1.5$, we find $V_{\text {direct }}=3.5 \times 10^{-7} \mathrm{eV}$ for $I=10^{7} \mathrm{~W} / \mathrm{cm}^{2}=10^{14} \mathrm{erg} /\left(\mathrm{s} \mathrm{cm}^{2}\right)$.

For the guest-host reorientational potential, consider the molecular structures of the dye guest $1 \mathrm{AAQ}$ and LC host $5 \mathrm{CB}$ in Chapter 2. Although the exact nature of the guest-host orientational interaction is still under question, we assume that dipole-dipole interaction between the amino group of the guest molecules and the nitrile group of the host molecules is the main mode of interaction. The orientational potential for such dipole groups is of the order $0.1 \mathrm{eV}$ per nearest-neighbor pair in the liquid state [39]. If each guest molecule has two LC nearest-neighbors, and the dipolar interaction beyond the nearest-neighbor is negligible, then with the typical doping concentration of 1 guest for 1000 host molecules, we have, on average, an orientational potential per LC molecule due to an oriented guest molecule $U_{\text {guest }}=0.1 \mathrm{eV} \times(2 / 1000)=2 \times 10^{-4} \mathrm{eV}$.

Now, the guest-host interactions are different for molecules in the ground and excited states. If $1 \%$ of the ground-state population is pumped into the excited state, and $U_{\text {guest }}$ of the ground and excited states of dye differ by $10 \%$, then the average guesthost reorientational potential exerted on a LC molecule is $V_{\text {guest }}=1 \% \times 10 \% \times U_{\text {guest }}=$ $2 \times 10^{-7} \mathrm{eV}$, comparable to $V_{\text {direct }}$ estimated above. This shows that indeed guest-host interaction could be strong enough to significantly enhance the optical reorientation of LC molecules.

In the case of a pulsed laser excitation, dye-induced LC reorientation mechanism is expected to decay away when the excited dye molecules return to the ground state and 
the anisotropy in the orientational distributions of the dye molecules diminishes towards thermal equilibrium. The dynamics of the dye molecules is then governed by the rotational diffusion equations under the simultaneous actions of optical excitation and spontaneous de-excitation. With a sufficiently short excitation pulse, the dynamics of dye relaxation basically evolves after the pulse is over. If the dye relaxation is much faster than the orientational relaxation of the LC molecules, then the reorienting effect of the dye guest on the LC host is essentially cumulative.

The above picture provides a qualitative description of the dye-induced enhancement effect on optical-field-induced reorientation of LC. Following this picture, we can now formulate the theory to provide a quantitative description of the effect. Note that in the physical model presented so far, no specific requirements need to be made for the phase of guest-host system. Thus it is clear that the physical principles presented above should apply whether the liquid crystal host is in the nematic or isotropic phase. Starting from the next section and for the rest of our work, we focus on the isotropic phase, for reasons described earlier in section 2.3.

\subsection{Formalism of Dynamic Equations}

The orientation of a rod-like molecule in the isotropic phase can be specified by a single angle $\theta$ formed by the long molecular axis and the field direction, and the timedependent molecular orientational distribution for the $i$-type molecules is described by the distribution function $f_{i}(\theta, t)$, which can be expanded into a series of Legendre polynomials 
$P_{l}$ as:

$$
f_{i}(\theta, t)=\frac{1}{4 \pi} \sum_{l=0,2,4, \ldots}(2 l+1) Q_{i}^{(l)}(t) P_{l}(\cos \theta)
$$

where

$$
Q_{i}^{(l)}(t)=\int f_{i}(\theta, t) P_{l}(\cos \theta) d \Omega
$$

are the Legendre moments of the distribution. Note that all odd- $l$ moments are zero due to the inversion symmetry of the system. Specifically, the zeroth-order moment $Q_{i}^{(0)}(t)=N_{i}(t)$ is the number density of the $i$-type molecular species and the second-order moment divided by the number density, $Q_{I}^{(2)}(t) / N_{i}(t)$, is the usual orientational order parameter. In our study we limit ourselves to the case of low optical pumping intensity, i.e. the induced orientational ordering is small, hence only $Q_{i}^{(2)}$ plays a role. We shall then simply write $Q_{i}$ for $Q_{i}^{(2)}$. The rotational dynamics of the system is described by the three functions $Q_{i}(t)$ for the three molecular species. An alternative and equivalent way to define $Q_{i}(t)$ is by:

$$
\begin{aligned}
Q_{i} & =\left\langle S_{i}\right\rangle / V \\
\text { with } S_{i} & =\sum_{m} s_{i}^{m} \equiv \sum_{m} \frac{1}{2}\left(3 \cos ^{2} \theta_{i}^{m}-1\right)
\end{aligned}
$$

where the sum is over the total number of the $i$-type molecules under consideration, $\theta_{i, m}$ is the angle between the long axis of the molecule and the optical field, $V$ is the volume of the sample, and the angular brackets denote the ensemble average. In an isotropic system, $f_{i}(\theta, t)$ has no $\theta$-dependence and $Q_{i}=0$.

The equations of motion for $Q_{i}$ originate from the rotational Brownian motion of the molecular species. In the limit where the rotational Brownian motion consists of small angular steps and can be approximated as a continuous diffusional process described by 
a rotational diffusion coefficient $D_{i}$, the distribution function $f_{i}(\theta, t)$ obeys the following Einstein-Schomulchowski equation:

$$
\frac{\partial}{\partial t} f_{i}-D_{i} \frac{1}{\sin \theta} \frac{\partial}{\partial \theta} \sin \theta\left(\frac{\partial}{\partial \theta}+\frac{1}{k_{B} T} \frac{\partial U_{i}}{\partial \theta}\right) f_{i}=W_{i}
$$

Here $k_{B} T$ is thermal energy, $W_{i}(\theta)$ is the rate of population transition, and $U_{i}(\theta)$ is the orientational potential energy affecting the rotational drift. The orientational potential consists of two contributions:

$$
U_{i}=U_{i}^{E M}+U_{i}^{i n t}
$$

representing, respectively, the direct torque exerted by the external electromagnetic field and the reorientational effect of intermolecular interactions. We will use Eq. (3.4) to derive the dynamical equations for the $Q_{i}$, as shown below.

\subsubsection{Dye response}

We consider first the dye molecules in the ground and excited states, $i=g$ and $e$, respectively. The rates of transitions into the ground and excited states are given by:

$$
W_{e}(\theta, t)=-W_{g}(\theta, t)=p(\theta) f_{g}(\theta, t)-\frac{f_{e}(\theta, t)}{\tau_{f}}
$$

where $p(\theta)=\left(3 \beta I \cos ^{2} \theta\right) /\left(h \nu N_{d}\right)$ is the pump excitation rate assuming that the transition dipole is parallel to the long molecular axis, $\nu$ and $I$ are the optical pump frequency and intensity, respectively, $\beta$ is the linear absorption coefficient, $h$ is the Planck's constant, $N_{d}$ is the total dye number density, and $\tau_{f}$ is the excited-state lifetime. Here we have assumed that stimulated emission is negligible, which is reasonable because of the large Stokes shift 
between the absorption and fluorescence spectra for the dye in study (Fig. 2.5). Also, we have assumed that the orientation of the excited dye molecule is unaffected by the various vibrational, radiative, and nonradiative decay processes that it undergoes as it decays back to the ground state. This assumption is based on the physical argument that these decay events happen too quickly to affect the orientation of the molecule. Experiments have in general validated this argument.

We next turn our attention to the orientational potential, $U_{i}=U_{i}^{E M}+U_{i}^{i n t}$ in Eq. (3.4), for $i=g$ and $e$. It turns out that we can neglect the optical torque on the dye molecules, the $U_{i}^{E M}$ term, because its effect in generating orientational anisotropy in the dye population is much smaller than that of the polarized optical excitation, the $W_{i}$ term. The $U_{i}^{\text {int }}$ term for the dye population is consisted of two parts: (i) the dye-dye interaction and (ii) the dye-host interaction. The former is negligible because of the low dye concentration used. Also, at the relatively low pump intensity used, host anisotropy, $Q_{h} / N_{h}$, is very small $\left(\leq 10^{-5}\right)$, hence the effect of host-dye interaction on the dye anisotropy is negligible. Thus $U_{i} \sim 0$ for $i=g$ and $e$ (see Appendix A for a more rigorous derivation of this result).

Then, the dynamical equations for $Q_{e}$ and $Q_{g}$ can be obtained readily from Eq. (3.4) by multiplying both sides by $P_{2}(\cos \theta)=\frac{1}{2}\left(3 \cos ^{2} \theta-1\right)$ and integrate over the solid angle, applying the approximations $Q_{i}^{(4)} \ll Q_{i}$ and $N_{e}, Q_{e}, Q_{g} \ll N_{d} \simeq N_{g}$ (because of the low excitation intensity). We find:

$$
\begin{aligned}
& \left(\frac{\partial}{\partial t}+\frac{1}{\tau_{D_{e}}}\right) Q_{e}=-\frac{Q_{e}}{\tau_{f}}+A I(t) \\
& \left(\frac{\partial}{\partial t}+\frac{1}{\tau_{D_{g}}}\right) Q_{g}=+\frac{Q_{e}}{\tau_{f}}-A I(t)
\end{aligned}
$$


where $\tau_{D i} \equiv 1 / 6 D_{i}$ is the rotational diffusion time of the $i$-type species, and $A \equiv 2 \beta / 5 h \nu$. The solutions of Eq. 3.7 for $Q_{e}$ and $Q_{g}$ are:

$$
\begin{aligned}
Q_{e}(t) & =\int_{-\infty}^{t} A I\left(t^{\prime}\right) e^{-\left(t-t^{\prime}\right) / \tau_{Q e}} d t^{\prime} \\
Q_{g}(t) & =\int_{-\infty}^{t}\left[\frac{Q_{e}\left(t^{\prime}\right)}{\tau_{f}}-A I\left(t^{\prime}\right)\right] e^{-\left(t-t^{\prime}\right) / \tau_{D_{g}}} d t^{\prime}
\end{aligned}
$$

with $\tau_{Q e} \equiv\left(1 / \tau_{D_{e}}+1 / \tau_{f}\right)^{-1}$. Eq. 3.8 completely describe the rotational dynamics of the dye molecules.

\subsubsection{Pure LC response}

We now turn to the discussion of $Q_{h}$ for the host LC molecules. In principle, a similar derivation starting from the rotational diffusion equation for the orientational distribution of the LC molecules, Eq. (3.4), would yield the equation of motion for $Q_{h}$. However, the situation here is complicated by the characteristically strong orientational interactions between neighboring LC molecules $\left(U_{h}^{\text {int }}\right.$ in Eq. (3.5)). In fact, it is this strong intermolecular orientational coupling that gives rise to the nematic phase of the LC as the temperature is lowered from the isotropic phase. The strong host-host interaction makes the mean-field approximation inadequate for describing the rotational dynamics of liquid crystals in the isotropic phase. This topic in fact has received considerable interests, both theoretically and experimentally, over the last three decades $[34,40,41,42,43,44,45,46$, $47,48,49,50,51,52,53,54,55,56,57,58,59,60]$. Though a complete understanding of the subject seems still missing $[58,59,60]$, many salient features have been understood.

The strong orientational coupling between LC molecules gives rise to local orienta- 
tional ordering which are usually visualized as microscopic pseudo-nematic domains existing in the macroscopically isotropic system, as described in section 2.1.2 [34]. Orientational fluctuation of these domains, or equivalently, randomization of their directors with respect to each other after a reorienting torque, gives rise to the well-known collective response, with exponential decay time in the range of nanoseconds and above. This collective response of LC is described well by the Landau-de Gennes theory treating the LC intermolecular interactions as a mean-field $[34,40,41,42,43,44,45,46,51,52]$. As the temperature is lowered towards the isotropic-nematic transition, the size of the pseudo-nematic domains, and thus the collective response decay time, tend to diverge, exhibiting characteristic pretransitional behavior. Beyond the mean-field approximation, or equivalently, beyond orientational relaxation through randomization of the pseudo-nematic domains with respect to each other, the interactions between the neighboring molecules result in a number of other modes of relaxation with much shorter orientational relaxation times, reflecting molecular orientational behavior from within the pseudo-nematic domains down to individual molecules. These relaxation modes exhibit no pretransitional behavior, and have been observed experimentally, both in the frequency domain [48] and time domain $[49,50,54,61,56,57]$.

The description of LC rotational dynamics with multiple orientational relaxation times was formulated earlier by Flytzanis and Shen [47] where the intermolecular interaction between LC molecules are taken into account beyond the mean-field by a perturbative calculation following Zwanzig's iterative approach [62]. This treatment, though at best is an approximation to the yet-to-be-found complete solution to the problem, is the only model available presently that provides an analytical expression for the LC rotational dynamics, 
and as will be shown later, explains our experimental data quite well. We briefly sketch their derivation here.

Reference [47] starts from Kubo's fluctuation-dissipation theory, where the induced anisotropy due to an optical field of frequency $\Omega$ is given by:

$$
\begin{aligned}
Q_{h}(\Omega) & =\frac{2 \Delta \alpha_{h}^{\prime}}{3 k_{B} T V}\left[\left\langle S_{h}(0) S_{h}(0)\right\rangle+i \Omega \int_{0}^{\infty}\left\langle S_{h}(0) S_{h}(t)\right\rangle e^{-i \Omega t} d t\right]\left|E_{l o c}\right|_{\Omega}^{2} \\
& \equiv \gamma(\Omega)\left|E_{l o c}\right|_{\Omega}^{2} .
\end{aligned}
$$

Here, $k_{B} T$ is the thermal energy and $E_{l o c}$ is the local field, which represents the overall reorienting field felt by an individual LC molecule. It is the sum of the applied field $E_{0}$ and the mean orienting field created by neighboring LC molecules. In terms of interaction energy, the total reorientational potential energy experienced by the $m$-th LC molecule is

$$
-\frac{2}{3} \Delta \alpha_{h}^{\prime}\left|E_{l o c}\right|_{\Omega}^{2} s_{h}^{m}=-\frac{2}{3} \Delta \alpha_{h}^{\prime} L^{2}\left|E_{0}\right|_{\Omega}^{2} s_{h}^{m}+U_{\mathrm{mf}}
$$

where $s_{h}^{m}$ is defined in Eq. (3.3) and $L$ is the usual Lorenz-Lorentz local field correction factor. The first term on the right-hand side is the molecule-field torque interaction and $U_{\mathrm{mf}}$ is the mean-field contribution from neighboring LC molecules. Note that $L$ and $U_{\mathrm{mf}}$ account for the local field created by the isotropic and anisotropic parts of the surrounding medium, respectively. (The right hand side of Eq. (3.10), apart from some numerical factors, is identical to $U_{h}$ in Eq. (3.4) - see Appendix A). Following Maier and Saupe [42], we have

$$
\begin{aligned}
U_{\mathrm{mf}} & =\left\langle U_{h h}>=-<\sum_{n} G_{h h}^{m n} s_{h}^{m} s_{h}^{n}\right\rangle=-\left\langle\sum_{n} G_{h h}^{m n}\right\rangle\left\langle S_{h}\right\rangle s_{h}^{m} \\
& =-\frac{u_{h h}}{3} \cdot \frac{Q_{h}}{N_{h}} \cdot s_{h}^{m} \text { with } u_{h h} \equiv 3\left\langle\sum_{n} G_{h h}^{m n}\right\rangle .
\end{aligned}
$$


Here $G_{h h}^{m n} s_{h}^{m} s_{h}^{n}$ denotes the orientational interaction energy between the $m$-th and $n$-th host molecules and the angle brackets denote the ensemble average. We then find:

$$
\Delta \alpha_{h}^{\prime}\left|E_{l o c}\right|_{\Omega}^{2}=\Delta \alpha_{h}^{\prime} L^{2}\left|E_{0}\right|_{\Omega}^{2}+\frac{1}{2} u_{h h} \frac{Q_{h}(\Omega)}{N_{h}}
$$

From Eqs. (9) and (12), we obtain

$$
Q_{h}(\Omega)=\frac{\gamma(\Omega)}{\left[1-\gamma(\Omega) u_{h h} / 2 \Delta \alpha_{h}^{\prime} N_{h}\right]} L^{2}\left|E_{0}\right|_{\Omega}^{2}
$$

In order to find $Q_{h}(\Omega)$ one needs to know $\gamma(\Omega)$, defined in Eq. (3.9). It is in the calculation of $\gamma(\Omega)$ that one needs to go beyond the mean-field approximation. In the calculation, the orientational distribution of the LC molecules is subjected to the diffusion equation, Eq. (3.4), with $W_{h}=0$ (no LC population transfer) and the intermolecular interaction $U_{h}^{\text {int }}$ given by the full microscopic form:

$$
U_{h}^{i n t}=-\sum_{m, n} G_{h h}^{m n} s_{h}^{m} s_{h}^{n} \quad m \neq n
$$

Then, in order to go beyond the mean-field approximation, Ref. [47], following Zwanzig's approach [62], used a series expansion of $U_{h}^{\text {int }}$ to handle the double summation to obtain $\gamma(\Omega)$. The details of this calculation is quite cumbersome, and we state only the result here. Ref. [47] found the result:

$$
Q_{h}(\Omega)=\frac{2 \Delta \alpha_{h}^{\prime} L^{2} N_{h}}{15 k_{B} T} \sum_{k} \frac{C_{k}^{\prime}}{1-i \Omega \tau_{k}}\left|E_{0}\right|_{\Omega}^{2}
$$

where the index $k$ denotes the order of terms in the series expansion, with $C_{k}$ describing their strengths and $\tau_{k}$ their orientational relaxation times. The $k=1$ term represents the meanfield contribution, with $C_{1}^{\prime}=T /\left(T-T^{*}\right)$ and $\tau_{1}=T / 6 D_{h}\left(T-T^{*}\right)$ where $T^{*} \equiv u_{h h} / 15 k_{B}$ is 
the fictitious second-order phase transition temperature (which is usually about $1 \mathrm{~K}$ below the actual nematic-isotropic phase transition temperature $T_{n i}$ ). Both $C_{1}$ and $\tau_{1}$ exhibit the characteristic pretransitional behavior. The $k \geq 2$ terms come from going beyond the mean-field approximation in the expansion, and represents more individual molecular characteristics, exhibiting no critical pretransitional behavior. Fourier-transform of Eq. (15) yields immediately the time-dependent equation for $Q_{h}$ :

$$
Q_{h}(t)=\frac{2 \Delta \alpha_{h}^{\prime} L^{2} N_{h}}{15 k_{B} T} \sum_{k} \frac{C_{k}^{\prime}}{\tau_{k}} \int_{-\infty}^{t}\left|E_{0}\right|^{2}\left(t^{\prime}\right) e^{-\left(t-t^{\prime}\right) / \tau_{k}} d t^{\prime}
$$

The above equation describes the rotational dynamics of the pure LC. If terms with $k \geq 2$ are neglected in Eq. (3.16), then it is identical to that obtained if the LC intermolecular interaction is described by a mean-field (see Appendix A).

\subsubsection{Dye-doped LC response}

With dye-doping of the LC, guest-host interaction is introduced in addition to the host-host interaction. Considering the low concentration of the dye, and as the lowest approximation, we treat the guest-host interaction as a mean-field. Then the orientational potential seen by the LC molecules now consists of not only $U_{h h}$, but also of $U_{h e}$ and $U_{h g}$ for interactions between the host LC molecules and the excited-state and ground-state dye molecules, respectively. Similar to $U_{h h}$ in Eq. (11), they are defined as

$$
U_{h e}=-\sum_{n} G_{h e}^{m n} s_{h}^{m} s_{e}^{n} \quad ; \quad U_{h g}=-\sum_{n} G_{h g}^{m n} s_{h}^{m} s_{g}^{n}
$$


We now have

$$
\begin{aligned}
U_{\mathrm{mf}} & \left.=<U_{h h}+U_{h e}+U_{h g}\right\rangle=-\sum_{i=g, e, h} \frac{u_{h i}}{3} \cdot \frac{Q_{i}}{N_{t}} \cdot s_{h}^{m}, \\
\text { with } \quad u_{h i} & \equiv \frac{3 N_{t}}{N_{i}}\left\langle\sum_{n} G_{h i}^{m n}\right\rangle,
\end{aligned}
$$

where $N_{t}=N_{h}+N_{e}+N_{g}$ being the total molecular density of the sample. Note that in our case of low dye-doping concentration, $N_{d} / N_{h} \simeq 1 / 2000$, so $N_{t} \simeq N_{h}$. The factor 3 in the definition of $u_{h i}$ above is to make it equivalent to that defined in Ref. [18]. We then find from Eq. (10)

$$
\Delta \alpha_{h}^{\prime}\left|E_{l o c}\right|_{\Omega}^{2}=\Delta \alpha_{h}^{\prime} L^{2}\left|E_{0}\right|_{\Omega}^{2}+\frac{1}{2} u_{h h} \frac{Q_{h}(\Omega)}{N_{t}}+\frac{1}{2} u_{h e} \frac{Q_{e}(\Omega)}{N_{t}}+\frac{1}{2} u_{h g} \frac{Q_{g}(\Omega)}{N_{t}}
$$

With the guest-host interaction taken into account through the mean-field, the rest of derivation of the equation of motion for $Q_{h}$ is the same as in the pure case. Accordingly, Eq. (16) becomes

$$
Q_{h}^{\text {dye-doped }}(t)=Q_{h}^{\text {pure }}(t)+Q_{h}^{\text {enh }}(t)
$$

with

$$
\begin{aligned}
Q_{h}^{\text {pure }}(t) & =\sum_{k}\left(\frac{C_{k}}{\tau_{k}}\right) \int_{-\infty}^{t} B I\left(t^{\prime}\right) \cdot e^{-\left(t-t^{\prime}\right) / \tau_{k}} d t^{\prime} \\
Q_{h}^{e n h}(t) & =\sum_{k}\left(\frac{C_{k}}{\tau_{k}}\right) \int_{-\infty}^{t}\left[u_{h e} Q_{e}\left(t^{\prime}\right)+u_{h g} Q_{g}\left(t^{\prime}\right)\right] \cdot e^{-\left(t-t^{\prime}\right) / \tau_{k}} d t^{\prime}
\end{aligned}
$$

where $B=4 \pi L^{2} \Delta \alpha_{h}^{\prime} N_{t} / n c, I(t)=(n c / 2 \pi)\left|E_{0}\right|^{2}(t)$ is the optical pump intensity, and $C_{k} \equiv C_{k}^{\prime} / 15 k_{B} T$. Equations (3.20) and (3.8) completely describe the rotational dynamics of the dye-doped LC system. 


\subsection{Transient and steady-state enhancement}

We can now discuss how excitation of dye molecules can affect the reorientation dynamics of LC molecules. In Eq. 3.20 we see that beside the optical torque on the LC molecules, represented by $B I(t)$, there are also torques that come from orientational anisotropies of dye molecules, i.e. the terms $u_{h e} Q_{e}(t)$ and $u_{h g} Q_{g}(t)$. The latter show that reorientation effect of $Q_{e}$ and $Q_{g}$ on the LC molecules is gauged by the mean-field potentials $u_{h e}$ and $u_{h g}$, respectively, exerted on the LC molecules by the dye molecules in the excited and ground states. From Eq. (8), we see that $Q_{e}(t)$ and $Q_{g}(t)$ have opposite signs, and depending on the relative magnitudes of the two, the extra torques can enhance or reduce the reorientation caused by the optical torque. In other words, there is a competition between the excited and ground-state dye population in reorienting the host LC molecules: $Q_{e}(t)$ wants to help align the LC molecules parallel to the optical field whereas $Q_{g}(t)$ wants to align them perpendicular to the optical field. For quantitative discussion of how dye molecules affect optical reorientation of LC, we define an enhancement factor $\eta(t)$ as

$$
\eta(t)=Q_{h}^{\text {dye-doped }}(t) / Q_{h}^{\text {pure }}(t)
$$

To have a feel of how the enhancement factor varies with time following pulsed laser excitation, we need to know the values of various time constants involved. As will be shown later, the relaxation times $\tau_{Q e}$ and $\tau_{D g}$ for the dye are in the range of several hundreds of picoseconds (ps), while $\tau_{k}$ 's for the LC range from picoseconds to tens of nanoseconds (ns). With the excitation pulse width of $\sim 15 \mathrm{ps}$, much shorter than the various dye relaxation times, we have $Q_{e}$ and $Q_{g}$ build up to $Q_{o}$ and $-Q_{o}$ near the end of the pulse 
with $Q_{o} \equiv A \int_{-\infty}^{\infty} I(t) d t$. Soon after the pulse is over, $Q_{e}$ and $Q_{g}$, following Eq. (8), decay as

$$
\begin{aligned}
& Q_{e}(t) \simeq+Q_{o} e^{-t / \tau_{Q_{e}}} \\
& Q_{g}(t) \simeq-Q_{o}\left[(1+r) e^{-t / \tau_{D_{g}}}-r e^{-t / \tau_{Q_{e}}}\right]
\end{aligned}
$$

where $r \equiv \tau_{Q_{e}} \tau_{D_{g}} /\left[\tau_{f}\left(\tau_{Q_{e}}-\tau_{D_{g}}\right)\right]$, and $t=0^{+}$is taken as the time right after the pump pulse is essentially over. The direct effect of $Q_{e}(t)$ and $Q_{g}(t)$ on $Q_{h}(t)$, as governed by Eq. (21), lasts as long as they are nonzero, but their cumulative effect on $Q_{h}(t)$ persists. The enhancement factor $\eta$ should start at 1 before the pump pulse arrives or before $Q_{e}$ and $Q_{g}$ come into play, then becomes greater or less than 1 as $Q_{e}$ and $Q_{g}$ evolve, depending on whether the excited state or the ground state of the oriented dye molecules dominates in aligning the host molecules, and finally approaches a steady-state value as $Q_{e}(t)$ and $Q_{g}(t)$ decay to zero. Knowing that the relaxation times for $Q_{e}(t)$ and $Q_{g}(t)$ are in the hundreds of ps range, we expect the steady-state value of $\eta$ will be reached at $\sim 1$ ns after the pulsed excitation.

Under the assumption that the excitation pulse width is much shorter than the dye relaxation times $\tau_{Q e}$ and $\tau_{D g}$, and taking $F=1$, we have from Eq.(21), for $t>>\tau_{k}$ for $k \geq 2$, the approximate result

$$
\begin{aligned}
Q_{h}^{\text {dye-doped }}(t) & \approx B C_{1} e^{-t / \tau_{1}}\left\{1+\frac{A}{B}\left[u_{h e} \tau_{Q e}\left(1-e^{-t / \tau_{Q e}}\right)\right.\right. \\
& \left.\left.-u_{h g} \tau_{D g}(1+r)\left(1-e^{-t / \tau_{D g}}\right)+u_{h g} \tau_{Q e} r\left(1-e^{-t / \tau_{Q e}}\right)\right]\right\}
\end{aligned}
$$


and accordingly

$\eta(t)=1+\left\{\frac{A}{B}\left[u_{h e} \tau_{Q e}\left(1-e^{-t / \tau_{Q e}}\right)-u_{h g} \tau_{D g}(1+r)\left(1-e^{-t / \tau_{D g}}\right)+u_{h g} \tau_{Q e} r\left(1-e^{-t / \tau_{Q e}}\right)\right]\right\}$.

For $t \gg \tau_{Q e}, \tau_{D g}$, the above expression reduces to the steady-state form:

$$
\eta(t) \rightarrow \eta_{\infty}=1+\frac{A}{B} u_{h e} \tau_{Q e}\left[1-\frac{u_{h g}}{u_{h e}} \cdot \frac{\tau_{D g}}{\tau_{D e}}\right]
$$

As can be seen from Eqs. (21) and (22), the same steady-state enhancement factor is obtained if the pump light is a continuous-wave source or is consisted of pulses much longer than $\tau_{Q e}$ and $\tau_{D g}$. In the experiment, we can obtain $\eta_{\infty}$ in two ways: by looking at $\eta(t)$ at time much larger than $\tau_{Q e}$ and $\tau_{D g}$ after a short pulse excitation, and by measuring $\eta$ with an optical pulse much longer than $\tau_{Q e}$ and $\tau_{D g}$. This is what we did in our experiment as will be described later.

It is seen from Eqs. (3.24) and (3.25) that $\eta$ is larger or smaller than 1 depends on the relative magnitude of $u_{h e} \tau_{D e}$ and $u_{h g} \tau_{D g}$, which are governed by the difference in how excited- and ground-state dye molecules interact with the surrounding host LC molecules. The first excited electronic state of the anthraquinone dye used in our study is known to be a charge-transfer state [35]. Dye molecules in this state are expected to have a stronger interaction with the cyanobiphenyl host molecules than those in the ground state. The guest-host interactions also make the rotational diffusion time of the excited state longer. Together, they yield an enhancement factor larger than 1.

Up to date, only quasi-steady-state investigations of the enhanced optical reorientation in dye-doped LC system, using laser pulses of width $\sim 10 \mathrm{~ns}$ or longer, have been 
carried out. They measured the quantity $\eta_{\infty}$. Their results agree with the prediction of the Jánossy's model, presented in this section. However a dynamic study of the system should provide a much more stringent test of the model because of the added complexity. A recent work [63] looked at the transient rotational response of the dye molecules within the LC host and found that $\tau_{D e}$ is significantly larger than $\tau_{D g}$, indicating a stronger excited dyehost interaction than the ground-state dye-host interaction. For a complete understanding of the guest-host interaction one ought to measure not only the dynamic response of the guest but more importantly, also the dynamic response of the host. In our study reported here, we designed and performed a set of measurements to probe the rotational dynamics of the dye-doped LC system, governed by Eqs. (3.8) and (3.20). We describe our overall experimental strategy in the following section.

\subsection{Overview of experimental strategy}

To experimentally investigate the rotational dynamics of the guest-host system, we employed several implementations of the optical pump/probe scheme, shown schematically in Fig. 3.1. In such an experiment, a linearly polarized pump beam is used to create the orientational anisotropies $Q_{i}$, followed by a time-delayed probe beam that measures the relaxation of the anisotropies and hence the rotational dynamics of the system [37, 64]. Denoting the polarization direction of the pump beam as $\hat{n}$, and the laboratory coordinates as $u, v=x, y, z$, the orientational ordering of the system is described by:

$$
Q_{i}^{u v}=\left[(3 / 2) n_{u} n_{v}-(1 / 2) \delta_{u v}\right] Q_{i}
$$




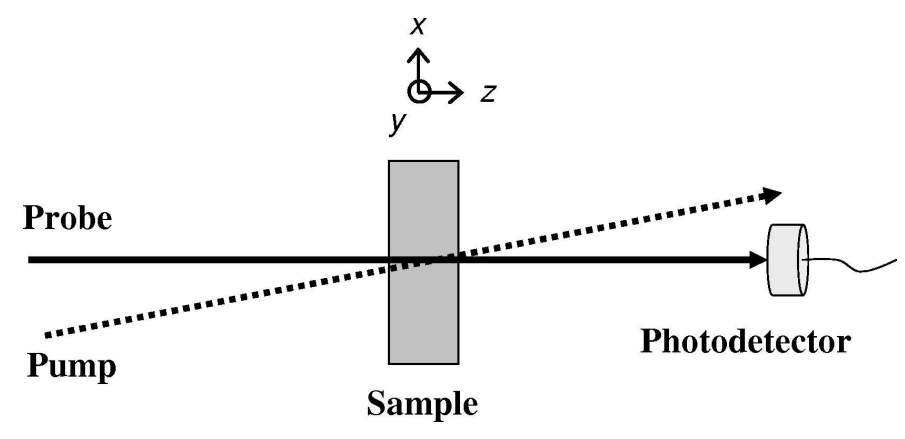

Figure 3.1: Schematic setup of a pump-probe experiment.

If $\hat{n}$ lies in the $\hat{x}-\hat{z}$ or $\hat{y}-\hat{z}$ plane, then the anisotropy seen by a probe pulse propagating along $\hat{z}$ is a difference in refractive index along the $\hat{x}$ and $\hat{y}$ directions:

$$
\begin{aligned}
\Delta n(t) & \equiv n_{x}-n_{y}=\Delta n^{\prime}+\imath \Delta n^{\prime \prime} \\
& =\frac{4 \pi L^{2}}{3 n_{o}} \sum_{i=g, e, h}\left(\Delta \alpha_{i}^{\prime}+\imath \Delta \alpha_{i}^{\prime \prime}\right)\left[Q_{i}^{x x}(t)-Q_{i}^{y y}(t)\right] .
\end{aligned}
$$

Here $n_{o}$ is the refractive index of the isotropic system, and $\Delta \alpha_{i}^{\prime}+\imath \Delta \alpha_{i}^{\prime \prime}$ is the complex polarizability anisotropy of the $i$-type molecular species at the probe wavelength. The $\Delta \alpha_{i}^{\prime}$ and $\Delta \alpha_{i}^{\prime \prime}$ terms in Eq. (28) describe, respectively, the induced birefringence and induced dichroism.

In order to quantitatively understand the guest-host orientational dynamics, as described by Eq. (20), we need to separately characterize the rotational dynamics of each of the three molecular species in the dye-doped LC system. We outline here the set of experiments that were carried out to achieve our goal. First, we performed a quasi-steadystate pump/probe measurement on the system to determine the steady-state enhancement factor $\eta_{\infty}$, and to look at the pump-wavelength dependence of the enhancement. Next 
we used transient fluorescence to determine the dye excited-state lifetime, $\tau_{f}$. Then we characterize the rotational dynamics of the dye responses by several pump/probe schemes, yielding $\tau_{f}, \tau_{Q e}$, and $\tau_{D g}$. Finally we adopted the pump/probe scheme of transient grating to look at the rotational dynamics of both the dye-doped LC and the pure LC systems. Knowing the relevant time responses of the dye molecules, we could fit the transient grating result and verify the model presented in the previous section.

This set of experiments will be described in the subsequent chapters. 


\section{Chapter 4}

\section{Quasi-steady-state reorientation of}

\section{dye-doped liquid crystals}

\subsection{Experimental setup}

We first measured the steady-state enhancement factor $\eta_{\infty}$ associated with the optical Kerr nonlinearity of the dyed-doped LC system, and its pump-wavelength dependence over the lowest absorption band of the dye at $\sim 475 \mathrm{~nm}$. The measured pump-wavelengthdependence of $\eta_{\infty}$ is a check on the physical picture of the model, since according to Eq. (25), $\left(\eta_{\infty}-1\right) \propto A$ is proportional to the absorption coefficient, but some previous wavelengthdependent measurements have found deviations from this proportionality $[12,65]$. The setup is shown schematically in Fig. 4.1, where pump beam $\mathrm{P}$ consists of wavelengthtunable $\sim 20$-nanosecond pulses from an optical parametric oscillator, which is pumped by a pulsed Q-switched Nd:YAG laser. The probe beam Pr comes from a $10 \mathrm{~mW}$ cw $\mathrm{HeNe}$ 
laser. The pump and probe beam have diameters of $3 \mathrm{~mm}$ and $0.5 \mathrm{~mm}$, respectively, and comes together at an angle of about $5^{0}$. The beams are arranged in the classic optical Kerr geometry: $\mathrm{P}$ and $\mathrm{Pr}$ are polarized along $\hat{y}$ and $\hat{x}+\hat{y}$ before the sample, respectively, and the analyzer transmission axis is set at the crossed position along $-\hat{x}+\hat{y}$. In the absence of the pump pulse, the analyzer completely blocks the probe beam. The pump pulse induces an optical anisotropy $\Delta n$ (Eq. (3.27)), causing the probe beam to leak through the analyzer and appear as the optical Kerr signal: $S \propto|\Delta n|^{2}$.

In this quasi-steady-state case, the excitation pulse width is $\sim 20 \mathrm{~ns}$, much longer compared to the dye time responses $\left(\tau_{D_{g}}, \tau_{Q_{e}} \leq 500 \mathrm{ps}\right.$ as will be shown later), while comparable or shorter than the longest LC time response ( $\tau_{1} \geq$ tens of ns). Then from Eqs. (3.20) and (3.8) we see that $Q_{h}$ accumulates over the length of the $\sim 20$-ns excitation pulse due to the long decay time $\tau_{1}$, while $Q_{e}$ and $Q_{g}$ do not accumulate because of the much shorter $\tau_{D_{g}}$ and $\tau_{Q_{e}}$. So the value of $Q_{e}$ and $Q_{g}$ is much smaller than $Q_{h}$ after the excitation is over. Thus the contribution to the optical anisotropy from $Q_{e, g}$ can be neglected. Then the optical Kerr signal from the experimental geometry in Fig. 4.1 is given by:

$$
S \propto\left[\Delta n^{\prime}\right]^{2} \propto\left[\Delta \alpha_{h}^{\prime} Q_{h}\right]^{2}
$$

Here only the birefringence contributes to the signal because the host $5 \mathrm{CB}$ is transparent at the HeNe wavelength of $633 \mathrm{~nm}$. To find the enhancement factor, Eq. (3.21), we need to compare the signals from the dye-doped and the pure LC samples under the same condition, particularly with the same excitation intensity. However, in the dye-doped case the pump intensity is attenuated as it traverses the sample due to absorption. To account for this, 


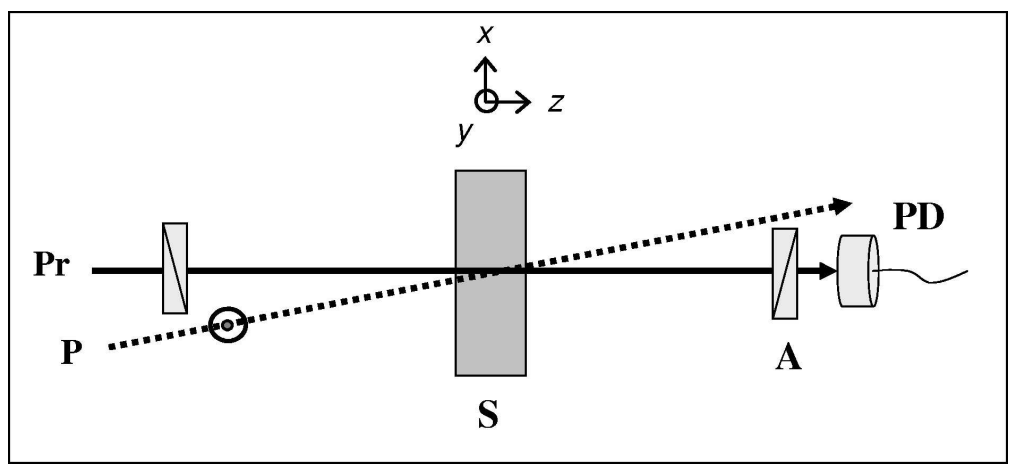

Figure 4.1: Top-view schematic setup of the quasi-steady-state experiment. Pr: probe beam, P: pump beam, S: sample; PD: photodetector. All beams lie on the $\hat{x}-\hat{z}$ plane, with $\mathrm{P}$ polarized along $\hat{y}$. Input polarization of $\mathrm{Pr}$ is set to be along $\hat{x}+\hat{y}$, and analyzer $\mathrm{A}$ is set at the crossed direction $-\hat{x}+\hat{y}$. Signal is from Pr leaking through analyzer A as the pump beams induce optical anisotropy in the sample S.

noting that $Q_{h}$ is linearly proportional to the intensity $I$, we normalize the signal by dividing it by the square of $\langle I\rangle$ which is the averaged intensity over the length $l$ of the sample. For the pure sample with no absorption, $\langle I\rangle_{\text {pure }}=I_{o}$, and for the dye-doped sample:

$$
\begin{aligned}
\langle I\rangle_{\text {dye-doped }} & \equiv(1 / l) \int_{0}^{l} I(z) d z=(1 / l) \int_{0}^{l} I_{o} e^{-\beta z} d z, \\
\text { yielding } \frac{\langle I\rangle_{\text {pure }}}{\langle I\rangle_{\text {dye-doped }}} & =\frac{\beta l}{1-e^{-\beta l}} .
\end{aligned}
$$

\subsection{Result and discussion}

In Fig. 4.2 we show the optical Kerr signal from the dye-doped and pure LC samples, as a function of input pump intensity $I_{o}$. The highest intensity corresponds to 


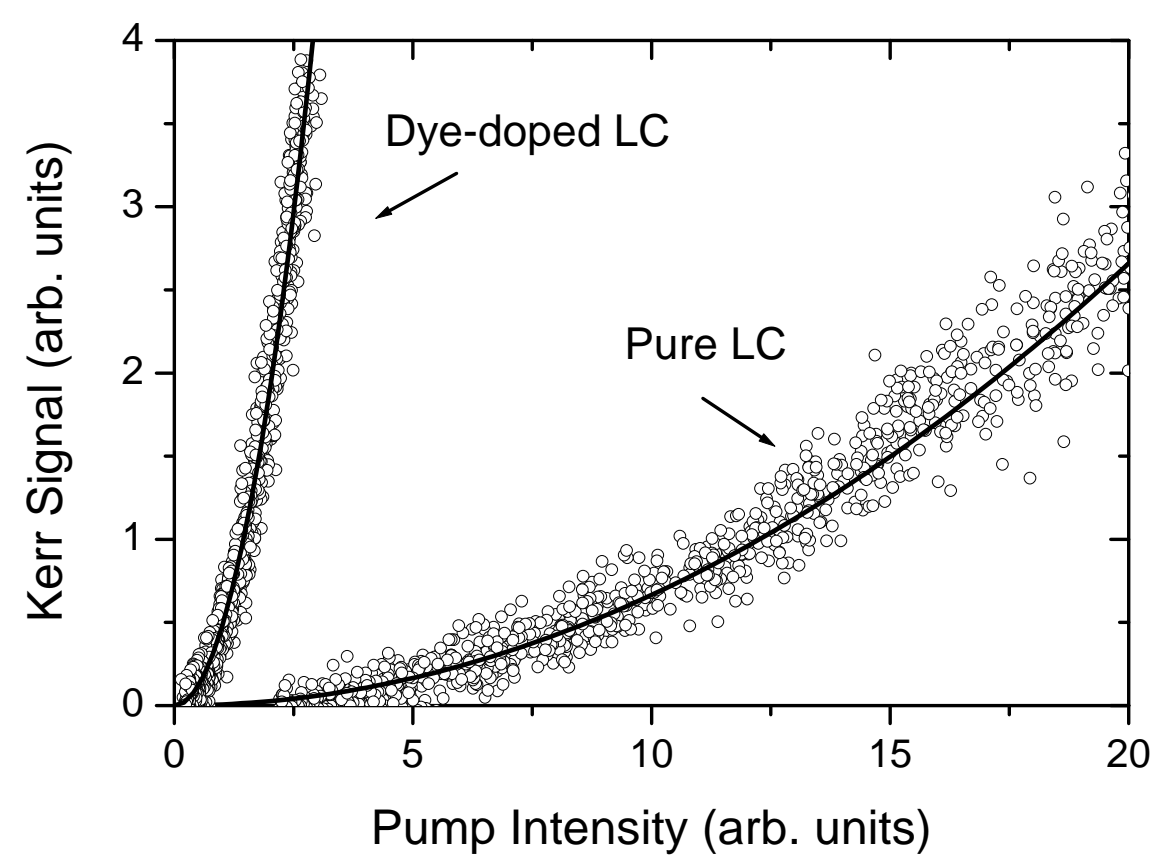

Figure 4.2: Optical Kerr signal versus pump intensity, from dye-doped and pure LC samples. Solid curves are quadratic fits.

pump energy $\simeq 5 \mathrm{~mJ} /$ pulse. The pump intensity and signal were measured shot-by-shot, and a single scan from highest to lowest intensity took about 10 minutes. At the same pump intensity we see that the signal from the dye-doped sample is much larger than that from the pure sample, demonstrating the enhanced optical reorientation of the Jánossy effect. Following Eq. (4.1) we fit the signal as a quadratic function of $I_{o}: S=D I_{o}^{2}$. Then the stead-state enhancement factor is given by:

$$
\eta_{\infty}=\frac{Q_{h}^{\text {dye-doped }}(t)}{Q_{h}^{\text {pure }}(t)}=\left[\frac{S_{\text {dye-doped }}}{S_{\text {pure }}} \frac{\langle I\rangle_{\text {pure }}^{2}}{\langle I\rangle_{\text {dye-doped }}^{2}}\right]^{1 / 2}=\frac{\beta l}{1-e^{-\beta l}}\left(\frac{D_{\text {dye-doped }}}{D_{\text {pure }}}\right)^{\frac{1}{2}}
$$

Figure 4.3 presents the factor $\left(\eta_{\infty}-1\right)$ measured at $\mathrm{T}=38^{\circ} \mathrm{C}$, and the linear absorption of the dye-doped LC, as functions of the pump wavelength. As expected, from 


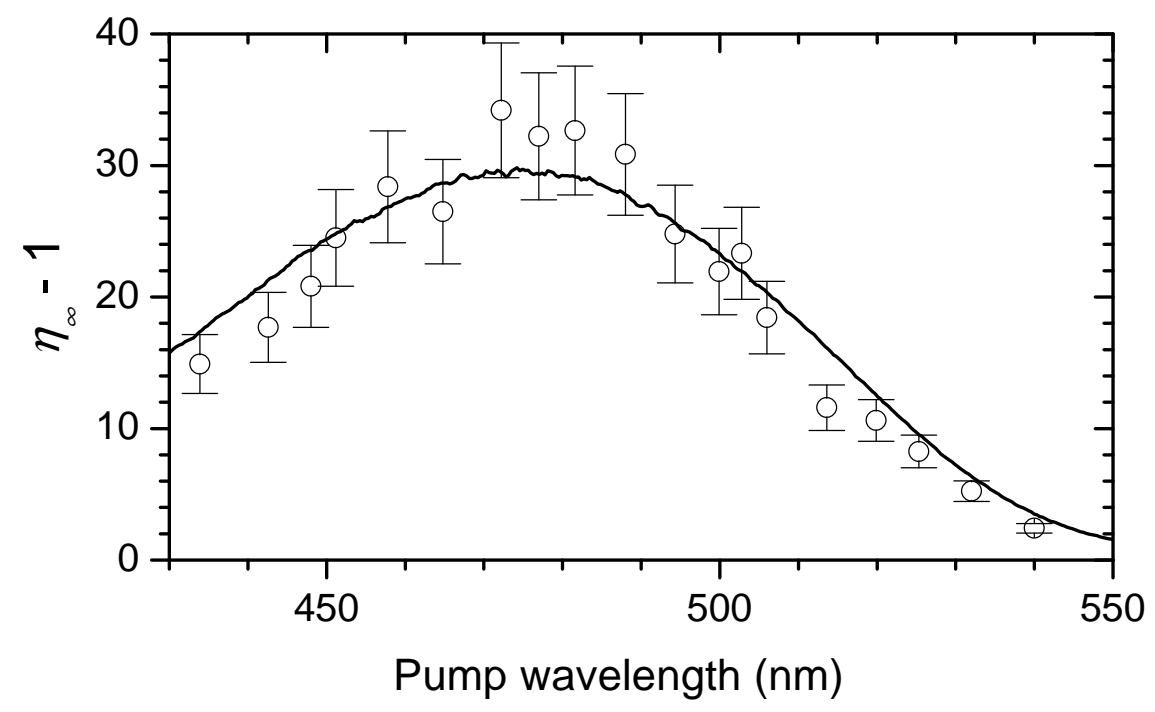

Figure 4.3: Steady-state enhancement factor versus pump wavelength. Solid line is the scaled absorption spectrum.

Eq. (3.26), $\left(\eta_{\infty}-1\right)$ follows the absorption spectrum. This confirms the picture that the enhancement comes from excitation of dye molecules into the charge-transfer state, agreeing with the result found in a recent study [66]. 


\section{Chapter 5}

\section{Dynamic response of dye molecules}

\subsection{Population decay: transient fluorescence}

We next embarked on measuring the dynamic response of the dye molecules, as characterized by the time constants $\tau_{f}, \tau_{Q e}$, and $\tau_{D g}$. We utilized a laser setup which at its core consists of a mode-locked Nd:YAG laser, giving $\simeq 20$-picosecond pulsed radiation at the fundamental wavelength of $1064 \mathrm{~nm}$. From this fundamental radiation, the second harmonic, third harmonic, and wavelengths tunable from the near-IR to UV are obtained through the use of nonlinear crystals and optical parametric processes.

To find the lifetime $\tau_{f}$ of the dye excited state, we utilized the transient fluorescence scheme where linearly-polarized picosecond-pulses at 532nm excited the dye molecules in the dye-doped LC sample, and the subsequent time-dependent fluorescence signal is monitored through an optical-Kerr shutter [67]. This time gate, refer to Fig. 5.1, consists of a 1-cm long Kerr cell filled with carbon disulfide, placed between a pair of crossed polarizers, with 


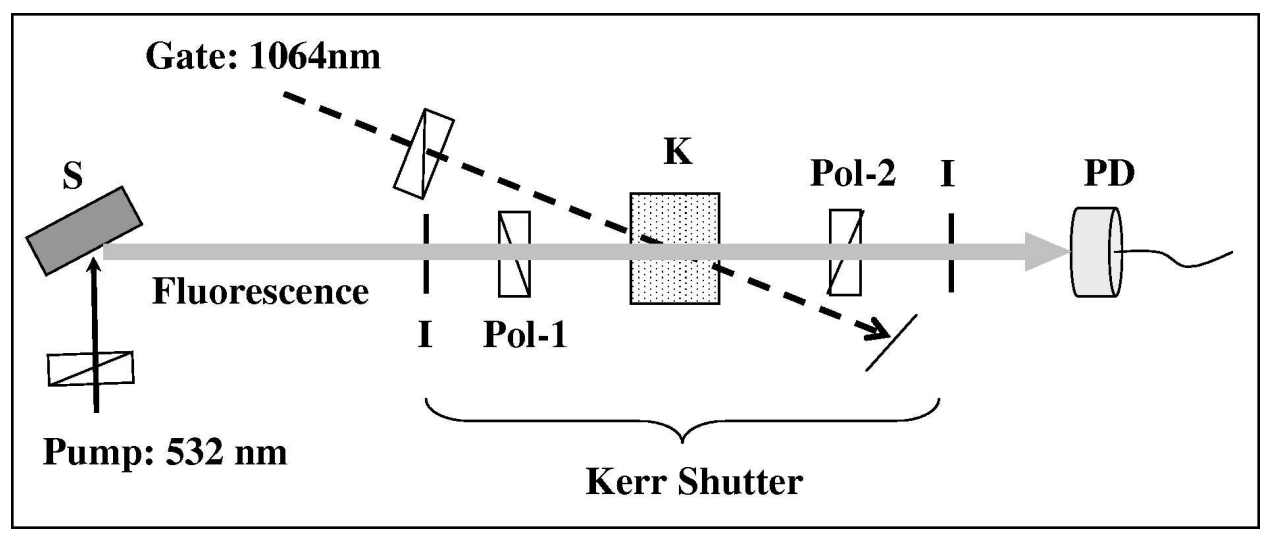

Figure 5.1: Schematic setup of transient fluorescence experiment. S: dye-doped LC sample, I: iris, Pol-1 and Pol-2: polarizers, K: Kerr cell filled with carbon disulfide, PD: photodetector.

irises at the two ends defining the direction of light allowed through the gate. With the polarizers set at the crossed positions, no fluorescence light will pass through the Kerr shutter. The time gating is achieved by sending in the 1064-nm pulse (from the same laser system) to overlap with the fluorescence light in the Kerr cell. The 1064-nm pulse will induce an optical Kerr effect in the Kerr cell to allow a fraction of the fluorescence to pass through the second polarizer and reach the photodetector (same principle as the experiment in Chapter 4). Carbon disulfide is known to have an optical Kerr response time $\simeq 2$ ps. Thus the fluorescence could pass through the gate only when the 20-ps 1064-nm pulse is inside the Kerr cell. So, by time-delaying the 1064-nm pulse with respect to the 532-nm excitation pulse, we could map out the time evolution of the fluorescence with resolution $\sim$ laser pulse width. The polarization of the fluorescence measured is controlled by varying the transmitted direction of the first polarizer with respect to the pump polarization direction, 


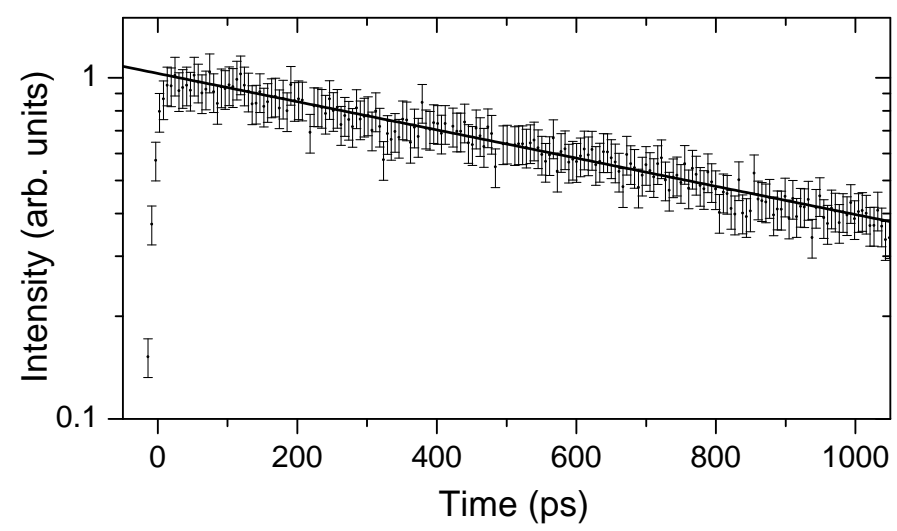

Figure 5.2: Fluorescence intensity, polarized at the magic angle with respect to the pump beam polarization versus time. Solid line is single exponential fit.

and adjusting the second polarizer accordingly to maintain the crossed condition.

The transient fluorescence technique is well-known and was described in detail in [68]. The fluorescence was measured at the "magic" polarization angle of $54.7^{\circ}$ with respect to the pump polarization so that it was independent of the orientational distribution of the excited molecules. It then yielded a single exponential decay with decay time $\tau_{f}[68]$. The result is shown in Fig. 5.2, from which we deduced $\tau_{f}=1050 \pm 60$ ps. In principle, if we measure separately fluorescence polarized parallel $\left(I_{\|}\right)$and perpendicular $\left(I_{\perp}\right)$ to the pump polarization, then since $\left[I_{\|}(t)-I_{\perp}\right] \propto e^{-t / \tau_{Q e}}, \tau_{Q e}$ could be deduced [68]. However, this procedure requires that $I_{\|}$and $I_{\perp}$ be properly normalized to each other, a task that is not easy to be accurate and could introduce large uncertainty in the deduced value of $\tau_{Q e}$. We therefore opted for a different method to find $\tau_{Q e}$, described in the next section. 


\subsection{Rotational diffusion: transient dichroism}

To probe the rotational dynamics of the dye both in the excited and ground states, we employed a heterodyned pump/probe scheme that measures transient dichroism. In the scheme, the dichroic part of the induced optical anisotropy can be probed separately from the birefringence part (Eq. (27)) [69, 70, 71]. It then allowed us to avoid complication due to induced birefringence in the $5 \mathrm{CB}$ host, which is transparent at pump and probe wavelengths and therefore does not contribute to the induced dichroism.

As depicted in Fig. 5.3, the pump pulse P1, incident on the sample at a small angle of $5^{\circ}$, was p-polarized in the $\hat{x}-\hat{z}$ plane and the probe pulse $\operatorname{Pr}$ was polarized along $\hat{x}+\hat{y}$. The two pulses had energies of 20 and 2-6 $\mu \mathrm{J}$ and beam diameters of 1 and $0.4 \mathrm{~mm}$, respectively. Pump wavelength was set at 480nm, near the absorption peak of the dye in the ground state. The analyzer A was set at a small heterodyning angle $\gamma$ away from the crossed position of $-\hat{x}+\hat{y}$. Note that with $\gamma \neq 0$, this geometry is different from the classic, non-heterodyned, optical Kerr geometry used in Chapter 4. With the current geometry, if the optical anisotropy is small, the heterodyned signal leaking through the analyzer is

$$
S_{t}(\gamma, t)=S_{o}\left\{\left[\frac{\pi l}{\lambda} \Delta n^{\prime \prime}(t)-\gamma\right]^{2}+\left[\frac{\pi l}{\lambda} \Delta n^{\prime}(t)\right]^{2}\right\}
$$

where $S_{o} \equiv S_{i n} e^{-4 \pi l n_{o}^{\prime \prime} / \lambda}$ with $S_{i n}$ being the incident probe intensity, $\lambda$ the probe light wavelength, and $l$ the sample thickness. Equation (5.1) is a parabolic function of $\gamma$ with $(\pi l / \lambda) \Delta n^{\prime}(t)$ and $(\pi l / \lambda) \Delta n^{\prime \prime}(t)$ describing the position of the minimum on the ordinate and the abscissa axes, respectively. Thus by measuring $S_{t}$ versus $\gamma$ and fitting to find the minimum position, as was done in Fig. 5.5a, we can determine the dichroism $\Delta n^{\prime \prime}$ separately 


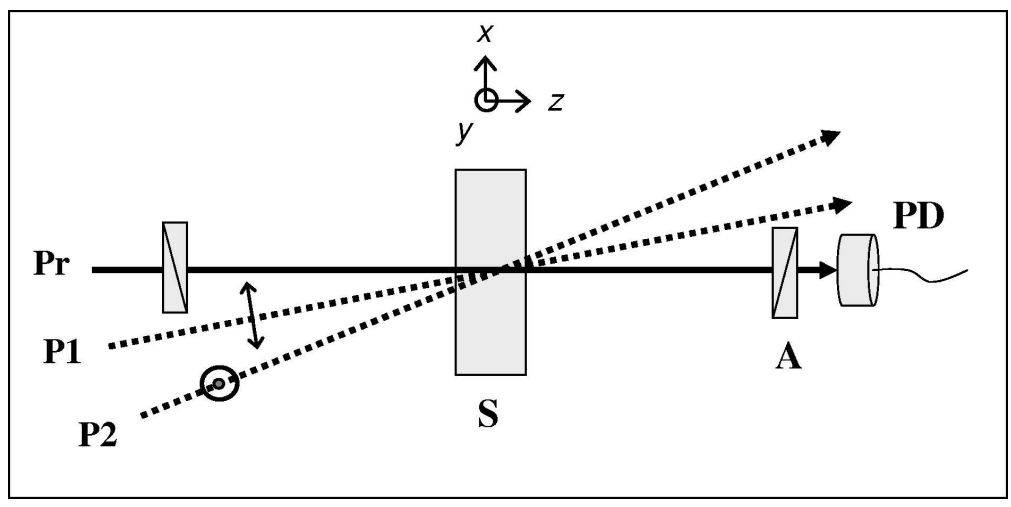

Figure 5.3: Top-view schematic of the experimental setups. Pr: probe beam; P1, P2: pump beams \#1 and \#2; S: sample; PD: photodetector. All beams lie on the $\hat{x}-\hat{z}$ plane, with P1 and P2 polarized as shown. Input polarization of $\mathrm{Pr}$ is set to be along $\hat{x}+\hat{y}$, and analyzer $\mathrm{A}$ is set at or near $-\hat{x}+\hat{y}$. Signal is from Pr leaking through analyzer A as the pump beams induce optical anisotropy in the sample S.

from the birefringence $\Delta n^{\prime}$.

To focus on the time-evolution of the induced dichroism, hence the rotational dynamics of the dye, we measured $S(t) \equiv S_{t}(-\gamma, t)-S_{t}(\gamma, t)$ and obtain:

$$
\begin{aligned}
S(t) & =S_{o} \frac{4 \pi l}{\lambda} \gamma \Delta n^{\prime \prime}(t) \\
& =\left(2 \gamma l S_{o} / 3 n_{o}\right) \sum_{i=g, e} \Delta \sigma_{i}\left[Q_{i}^{x x}(t)-Q_{i}^{y y}(t)\right]
\end{aligned}
$$

where $\Delta \sigma_{i} \equiv \sigma_{i, \|}-\sigma_{i, \perp}=\left(8 \pi^{2} / \lambda\right) \Delta \alpha_{i}^{\prime \prime}$ denoting anisotropy in the absorption cross-section of dye molecules in state $i$, with the parallel direction referring to the long molecular axis. Note that the induced dichroism could be positive or negative. An example is shown in Fig. 5.5b. 
Equation (5.2) shows that if there is a probe wavelength range where $\Delta \sigma_{e}$ or $\Delta \sigma_{g}$ is negligible compared to the other, then the state-specific dichroism, and hence the corresponding rotational dynamics, of the other can be measured. It is often possible to find at selected wavelengths that $\Delta \sigma_{g} \ll \Delta \sigma_{e}$, but not the reverse. Then probing the excitedstate rotational dynamics is straightforward, but not so for the ground state. One might think that the ground-state rotational dynamics could be deduced from the measured $S(t)$ of Eq. (5.2) at a wavelength where both $\Delta \sigma_{e}$ and $\Delta \sigma_{g}$ are nonvanishing if the excited state rotational dynamics and $\Delta \sigma_{e} / \Delta \sigma_{g}$ are known. Unfortunately, determination of $\Delta \sigma_{e} / \Delta \sigma_{g}$ is often complicated by model dependence and simultaneous contributions from absorption and stimulated emission to $\Delta \sigma_{e}$, yielding scattered results as found in the literature [72, 73]. Thus in order to measure the ground-state rotational dynamics, we must eliminate the contribution from the excited state in the induced dichroism measurement. We have devised a method employing two pump pulses to accomplish this.

As shown in Fig. 5.3, following the first pump pulse $\mathrm{P} 1$ at $t=0$, at $t=t_{D}$ we irradiate the sample with a second pump pulse P2, polarized perpendicularly to P1 and having the same wavelength. Our method is demonstrated qualitatively in Fig. 5.4. With P1 polarized along $\hat{n}$ in the $\hat{x}-\hat{z}$ plane, its $x$-component will create anisotropy along the $\hat{x}$ direction: $+Q_{o}$ in the excited state and $-Q_{o}$ in the ground state, represented by the solid and dotted double-headed gray arrows, respectively, in Fig. 5.4. After P1, the excited and ground-state anisotropies evolve following the dynamics specific to each state. If the rotational diffusion rate is different in the two states, e.g. $\tau_{D g}<\tau_{D e}$ shown in Fig. 5.4, then a net difference in the anisotropy magnitude would develop between the two states as time 


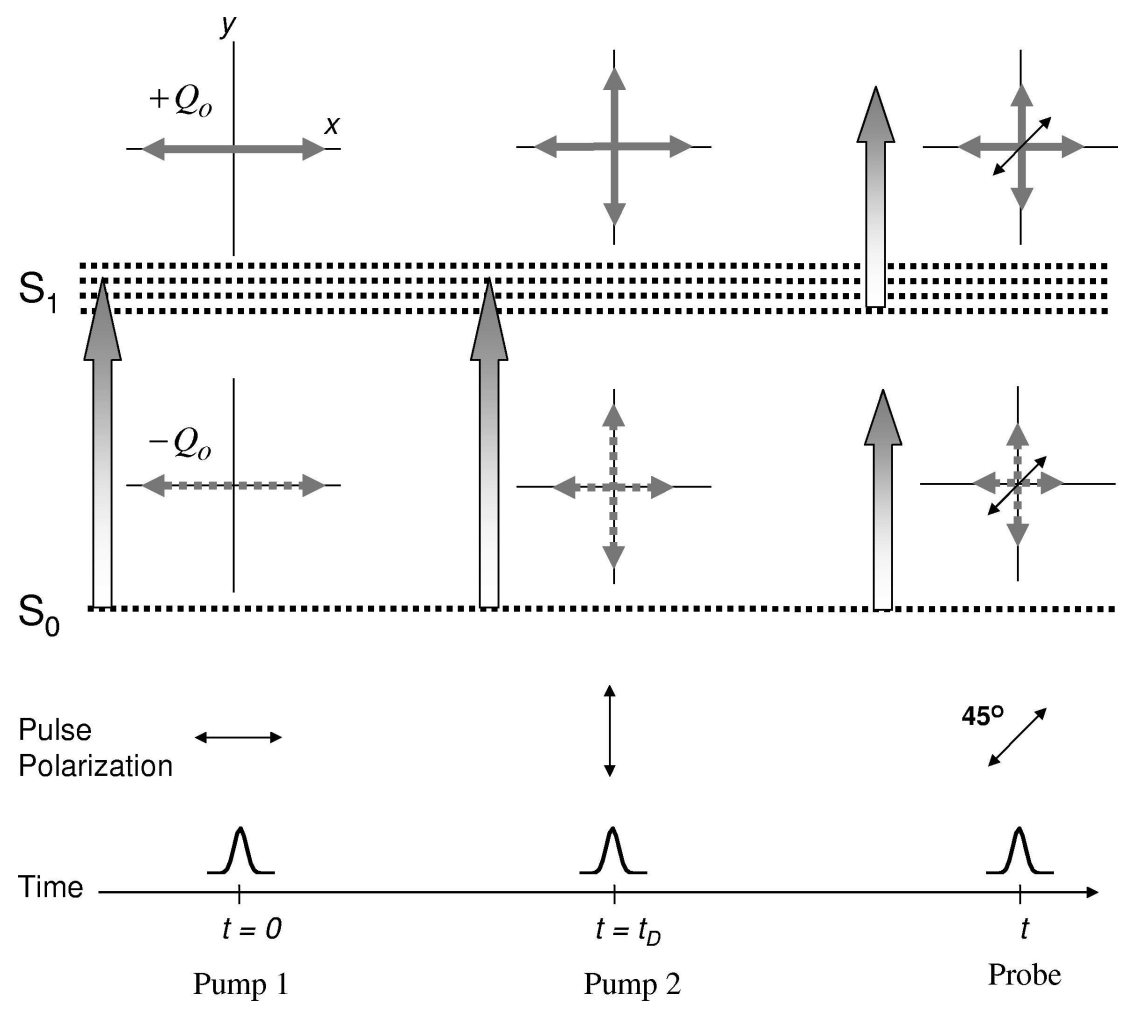

Figure 5.4: Schematic demonstration of the two-pump/probe technique to measure ground state rotational dynamics. Wavelength of pump pulses 1 and 2 is in resonance with the $\mathrm{S}_{o}-\mathrm{S}_{1}$ transition. Wavelength of probe pulse is varied to measure state-specific rotational dynamics. Pulse polarization shown is along the $x-y$ plane. See text for explanations.

proceeds. This is represented in Fig. 5.4 by the shortening of the double-headed arrows by different amount. At time $t_{D}$ after P1, the second pump pulse $\mathrm{P} 2$ of the same wavelength with $\mathrm{P} 1$, with its polarization along $\hat{y}$ and its intensity properly adjusted, would generate in the excited state an anisotropy along $\hat{y}$ that is equal in magnitude with the anisotropy along $\hat{x}$ created by $\mathrm{P} 1$, therefore nullifying any optical anisotropy that could be observed with a third time-delayed probe pulse polarized along $\hat{x}+\hat{y}$. P2 will not nullify the optical 
anisotropy coming from the ground state, because at time $t_{D}$ a difference in magnitude has developed between the excited state and ground state anisotropy created by P1 (due to the different rotational diffusion rates). Subsequently the probe pulse will see an optical anisotropy coming exclusively from the ground state. Furthermore, since the excited state population has equal anisotropies along $\hat{x}$ and $\hat{y}$, depopulation will add oriented molecules equally along $\hat{x}$ and $\hat{y}$ to the ground state, hence the net anisotropy of the ground state is unaffected by level decay. Its decay is governed exclusively by ground-state rotational diffusion. Therefore, the probe pulse could map out the intrinsic rotational diffusion of the ground state.

Quantitatively, the induced anisotropy in the excited state of the dye molecules due to P1 with intensity $I$ is $\left(Q_{e}^{x x}-Q_{e}^{y y}\right)_{t<t_{D}}=(3 / 2)(\hat{n} \cdot \hat{x})^{2} Q_{o} \cdot \exp \left(-t / \tau_{Q e}\right)$. To nullify this, P2 is polarized along $\hat{y}$ and has intensity $=I\left(n_{x}^{2} / n_{y}^{2}\right) \cdot \exp \left(-t_{D} / \tau_{Q e}\right)$, which then, according to Eqs. (3.22) and (3.26), would yield a net ground state anisotropy given by

$$
\begin{aligned}
{\left[Q_{g}^{x x}-Q_{g}^{y y}\right]\left(t>t_{D}\right)=} & (3 / 2)(\hat{n} \cdot \hat{x})^{2} Q_{o}(1+r) e^{-t_{D} / \tau_{Q e}} \\
& \times\left\{1-\exp \left[t_{D}\left(\tau_{D g}-\tau_{Q e}\right) / \tau_{D g} \tau_{Q e}\right]\right\} \cdot e^{-\left(t-t_{D}\right) / \tau_{D g}} .
\end{aligned}
$$

Then from Eq. (5.2), the observed rotational dynamics after P2, described by $S\left(t>t_{D}\right)$, is a single exponential decay with the ground state rotational diffusion time $\tau_{D g}$.

The results of our transient dichroism measurements are presented in Figs. 5.5 and 5.6. The dichroism signal was normalized pulse-by-pulse against the probe pulse energies, and the fluctuations of the pump were limited to less than $12 \%$. Each data point shown was the result of averaging over 100 pulses. 

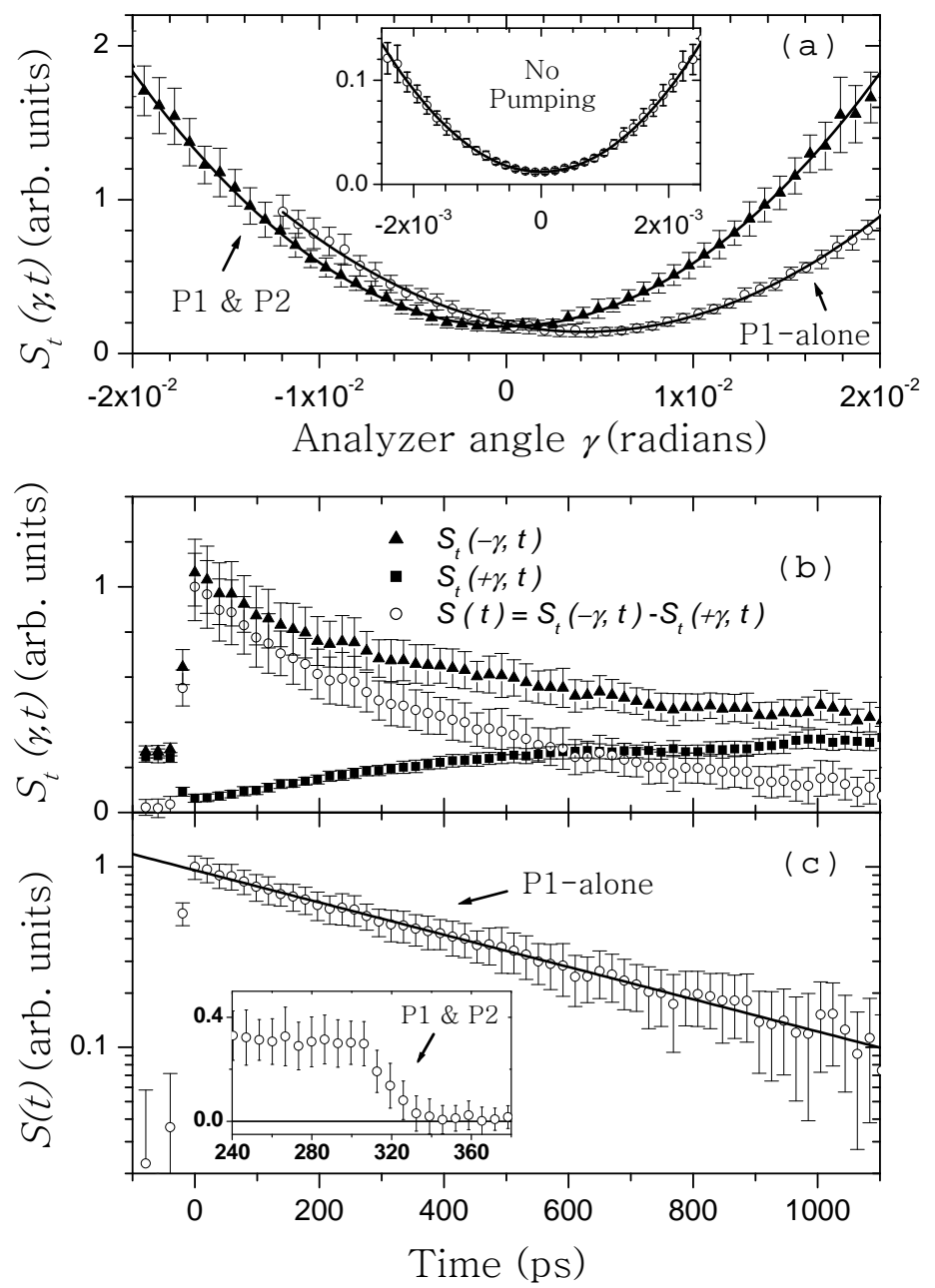

Figure 5.5: Dye excited-state dichroism probed at 600nm. (a) $S_{t}(\gamma, t)$ versus $\gamma$ at $t=340$ ps, induced by pump pulse P1 alone (at $t=0$ ) and by pump pulse $\mathrm{P} 1$ followed by pump pulse P2 (at $t=320 \mathrm{ps)} \mathrm{that} \mathrm{reduces} \mathrm{the} \mathrm{excited} \mathrm{state} \mathrm{dichroism} \mathrm{to} \mathrm{zero.} \mathrm{The} \mathrm{dichroism}$ is determined from the abscissa of the fitted parabolic minimum, which appears at $\gamma=0$ in the absence of the pump pulse (inset). (b) Transient dichroism signal $S(t)$ measured by taking the difference between $S_{t}(-\gamma, t)$ and $S_{t}(+\gamma, t)$. (c) Single exponential fit of the same $S(t)$ shown in (b). Inset shows how the dichroism induced by $\mathrm{P} 1$ is reduced to zero by $\mathrm{P} 2$. 
To selectively probe the excited-state rotational dynamics of $1 \mathrm{AAQ}$, the probe wavelength was set at $600 \mathrm{~nm}$, outside of the ground state absorption band (Fig. 2.6). Then the induced dichroism by $\mathrm{P} 1$ alone comes exclusively from excited state anisotropy and should decay exponentially with a time constant of $\tau_{Q e}$. The experimental result is displayed in Fig. 5.5b and 5.5c, from which we deduce $\tau_{Q e}=490 \pm 30$ ps. Knowing $\tau_{f}=1050 \pm 60 \mathrm{ps}$ from the fluorescence measurement, we find $\tau_{D e}=900 \pm 110 \mathrm{ps}$, agreeing within experimental error with the value reported by Paparo et al. [74].

We also tuned the probe wavelengths to the near-IR (also outside of the ground state absorption band) and measured the relative magnitudes of the excited absorption anisotropy at three different wavelengths, obtaining: $\Delta \alpha_{e}^{\prime \prime}(925 \mathrm{~nm}) / \Delta \alpha_{e}^{\prime \prime}(848 \mathrm{~nm}) \simeq 0.4$, $\Delta \alpha_{e}^{\prime \prime}(1024 \mathrm{~nm}) / \Delta \alpha_{e}^{\prime \prime}(848 \mathrm{~nm}) \simeq 0$, with uncertainty at about $15 \%$. These results will be used to corroborate our fitting of the overall optical anisotropy of the guest-host system, as described in the next chapter.

At all possible wavelengths to probe the ground-state dichroism of the dye 1AAQ, the excited-state absorption is significant. Thus we used the two-pump-pulse scheme described earlier to eliminate the excited state contribution. With the probe wavelength set at $600 \mathrm{~nm}$, we monitored the excited-state anisotropy to adjust the intensity of second pump beam P2 with respect to first pump beam P1. Fig. 5.5a shows the signal $S_{t}(\gamma, t)$, at $t=340$ ps, as a function of the heterodyning angle $\gamma$ (Eq. (5.1)), for the two cases of P1 alone and $\mathrm{P} 1$ followed by P2 at $t_{D}=320 \mathrm{ps}$. Recall that $S_{t}$ is a parabolic function of $\gamma$ with the abscissa of the minimum determined by the dichroism. With no pumping the minimum is at $\gamma=0$. With pump $\mathrm{P} 1$ alone, the minimum is at $\gamma=4.2 \pm 0.16 \times 10^{-3} \mathrm{rad}$; with $\mathrm{P} 1$ 
followed by P2, adjusted to the proper intensity, the minimum is at $\gamma=0 \pm 1.5 \times 10^{-4} \mathrm{rad}$. Thus we see that the excited state dichroism is nullified by P2 to better than $\pm 4 \%$ soon after $t=t_{D}$.

Then with the same sequential P1 and P2 pulses, we could probe the ground state dichroism using probe pulses at 505nm, which is inside the ground state absorption band. The result is shown in Fig. 5.6. We see that for $t<t_{D}$, the induced dichroism is negative as it is dominated by oriented ground-state bleaching, and for $t>t_{D}$, pulse P2 reversed the sign of the dichroism, as expected from Eq. (5.3) for $\tau_{D g}<\tau_{D e}$. The exponential decay of the dichroism after $t_{D}$ yields $\tau_{D g}=170 \pm 20 \mathrm{ps}$

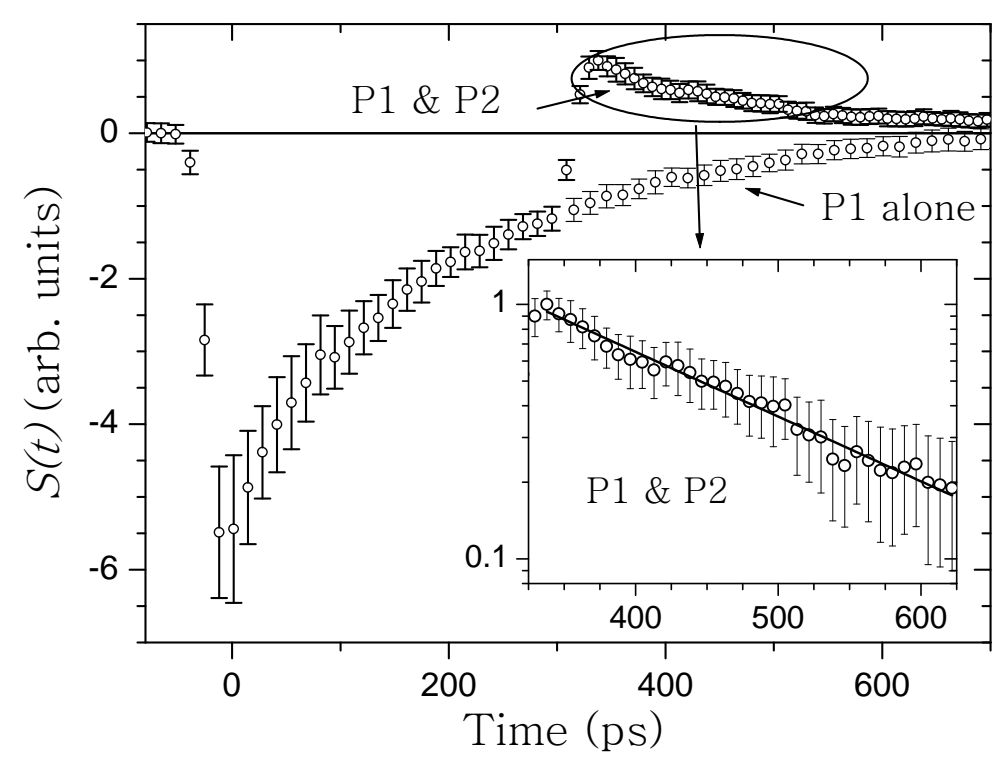

Figure 5.6: Transient dichroism probed at $505 \mathrm{~nm}$, induced by pump pulses $\mathrm{P} 1$ at $t=0$ ps and P2 at $t=320$ ps. After P2, the observed dichroism reverses sign and decays exponentially. Inset shows the single-exponential fit of the experimental data after P2. 
Summarizing the results of our measurements on the rotational dynamics of $1 \mathrm{AAQ}$ in $5 \mathrm{CB}$, we have found that for the excited state of $1 \mathrm{AAQ}, \tau_{f}=1050 \pm 60 \mathrm{ps}, \tau_{Q e}=490 \pm 30$ ps, and $\tau_{D e}=900 \pm 110 \mathrm{ps}$, and for the ground state, $\tau_{D g}=170 \pm 20 \mathrm{ps}$. The rotational diffusion time of the excited state is markedly longer than that of the ground state, signifying a stronger guest-host frictional force upon dye excitation, as have been found in previous state-specific rotational dynamics studies of various guest/host systems $[63,75,76,77,78$, $79,80]$. As seen through Eq. (3.25), the longer rotational diffusion time of the excited dye molecules contributes to a positive enhancement of the field-induced reorientation of the LC host molecules. Thus the result of our rotational dynamics measurements on $1 \mathrm{AAQ}$ in $5 \mathrm{CB}$ provide an independent confirmation of the change of guest-host interaction upon excitation of the guest that affects the light-induced reorientation of the host molecules, the central assumption of the current model explaining the Jánossy effect. 


\section{Chapter 6}

\section{Reorientational dynamics of}

\section{dye-doped liquid crystals}

\subsection{Experimental setup}

With the rotational dynamics of the dye characterized, we then proceeded to probe the overall rotational dynamics of the guest-host system using the pump/probe scheme of transient grating $[81,82]$. A schematic of the setup is shown in Fig. 6.1. We utilized the same laser system as described in the previous chapter. Two picosecond pump pulses, $\mathrm{P} 1$ and $\mathrm{P} 2$, at $532 \mathrm{~nm}$ with $s$ and $p$ polarizations, respectively, and each with energy of $40 \mu \mathrm{J} /$ pulse, overlapped at an angle of $14^{\circ}$ over a spot of $\sim 3.5 \mathrm{~mm}$ in diameter on the sample and formed a polarization grating that induced a transient refractive index grating in the medium. The time-delayed $s$-polarized probe pulse Pr with wavelength $\lambda_{\text {probe }}$, beam diameter of $1 \mathrm{~mm}$, and energy of $1 \mu \mathrm{J} /$ pulse was used to probe the transient grating by 


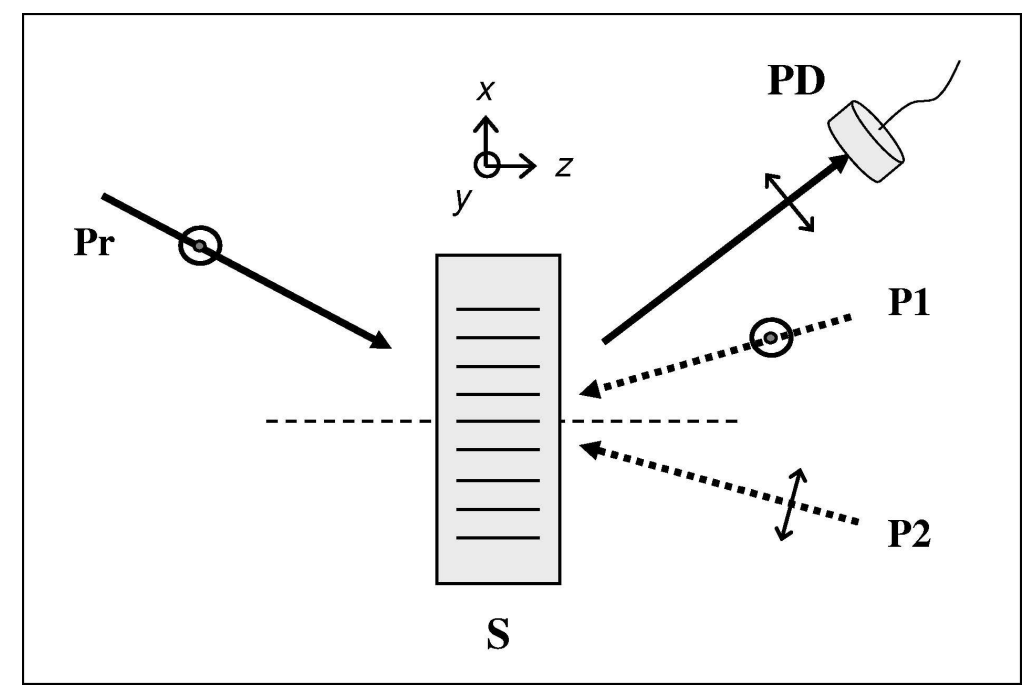

Figure 6.1: Top-view schematic of the transient grating setup. Pr: probe beam; P1, P2: pump beams \#1 and \#2; S: sample; PD: photodetector. All beams lie on the $\hat{x}-\hat{z}$ plane, with polarizations as shown. P1 and P2 overlap spatially and temporally at sample S, forming a polarization grating which diffracts $s$-polarized $\operatorname{Pr}$ as the $p$-polarized signal at the Bragg angle.

Bragg diffraction and the $p$-polarized diffracted output $S(t)$ was recorded. Pump and probe pulse energies were monitored for every laser shot, and each data point was the result of averaging about 100 pulses, where the signal was normalized pulse-by-pulse against the probe energy and the pump energy fluctuation kept within $\pm 12 \%$.

It is known that the diffracted signal from a grating of complex refractive index is given by [82]: $S \propto|\delta n|^{2}$, where $\delta n$ is the contrast between the minima and maxima of the complex refractive index grating. Because the field intensity is uniform across the polarization grating and only the polarization of the field varies, such a grating produces no thermal 
or acoustic effects normally associated with intensity gratings which could complicate data analysis. The contrast $\delta n$ is directly proportional to $\Delta n$ given in Eq. (3.27). Hence, with $I_{p r}(t)$ being the time-varying intensity of the probe pulse, the time-dependent diffraction signal is:

$$
S(t) \propto \int_{-\infty}^{\infty} I_{p r}\left(t-t^{\prime}\right) \cdot\left|\Delta n\left(t^{\prime}\right)\right|^{2} \cdot d t^{\prime}
$$

Our goal is to observe the reorientation of the host LC molecules due to the optical pulse with and without the presence of the dye. Thus we want to minimize the contribution of the dye to the overall signal by choosing appropriate probe wavelengths to minimize $\Delta \alpha_{g}$ and $\Delta \alpha_{e}$ in Eq. (3.27). We therefore set the probe-wavelengths to be in the near-IR range, away from the resonant transitions of the dye from the ground and excited states.

\subsection{Result}

The observed signal from a pure LC sample at $\lambda_{\text {probe }}=848 \mathrm{~nm}$ and a dye-doped sample at $\lambda_{\text {probe }}=848,925$, and $1024 \mathrm{~nm}$ are displayed as the square-root of $S$ versus $t$ (for convenience of visualizing $\Delta n(t)$ versus $t$ approximately) in Fig. 6.2. The time variation of $S$ from the pure LC sample were the same for the three $\lambda_{\text {probe }}$ 's, and the amplitude of the dye-doped signals shown in Fig. 7 have been normalized against that of the pure sample at the same probe wavelengths. The signals from the dye-doped sample have also been corrected for attenuation of the pump beam as it traverses the sample (procedure described in Chapter 4).

To characterize the response of our setup, we used a sample of benzene, whose 


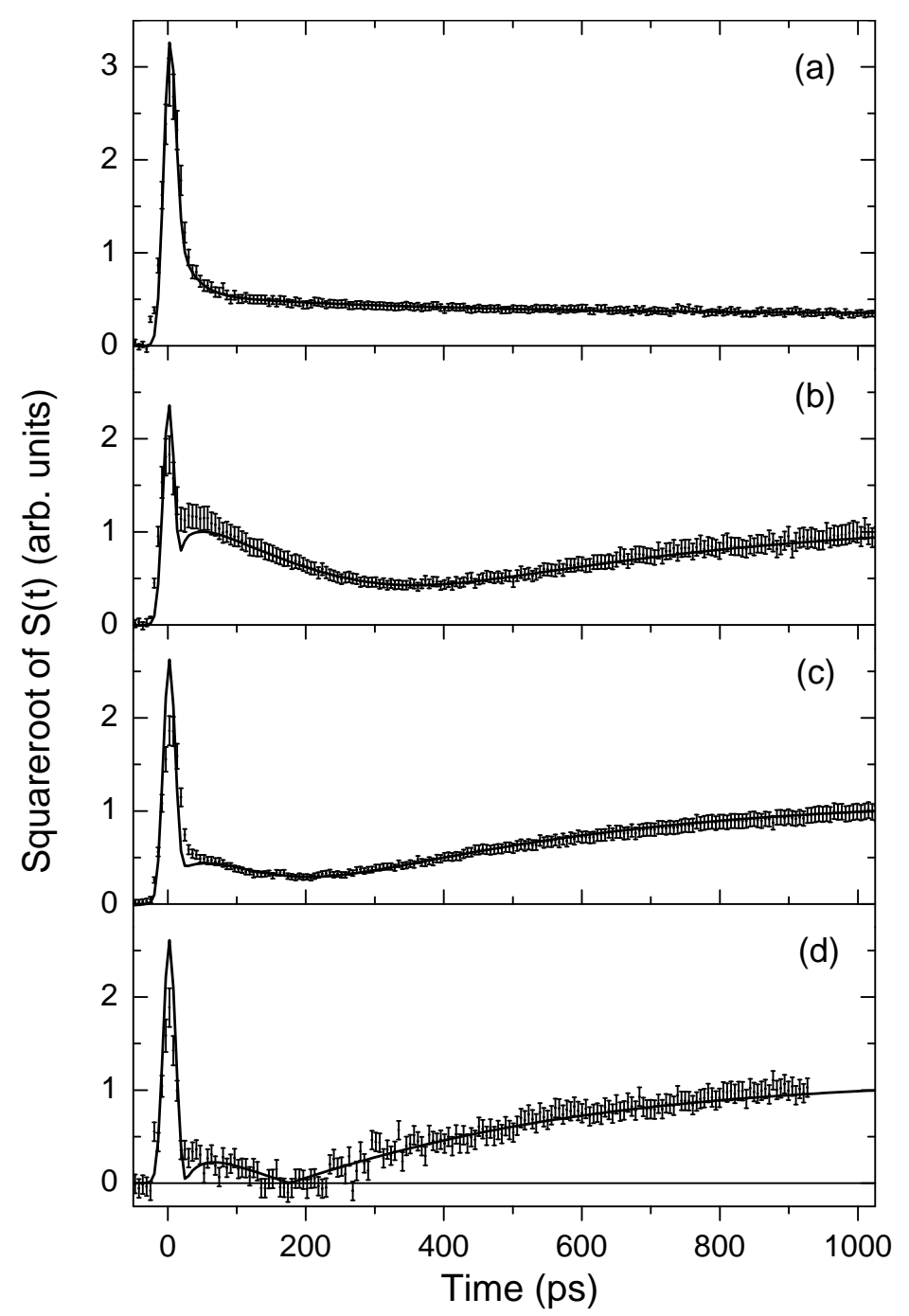

Figure 6.2: Result of the transient grating experiment from (a) pure LC at probe-wavelength of $848 \mathrm{~nm}$ and from dye-doped LC at (b) $848 \mathrm{~nm}$, (c) $925 \mathrm{~nm}$, and (d) $1024 \mathrm{~nm}$. Solid lines are theoretical fits. The signal from dye-doped LC are fitted with $\eta_{\infty}=3.3$ and $r_{u}=0.6$. See text for fitting procedure.

response time of $\sim 1 \mathrm{ps}$ [83] could be approximated as being instantaneous in our case. Taking the same Gaussian time profile for both the pump and probe pulses, fitting of the 
transient signal from benzene according to Eq. (6.1) yielded a full-width-half-maximum pulse width of $14 \mathrm{ps}$ for the pump and probe pulses. Comparing the signal from pure LC with that from benzene and knowing the nonlinearity of benzene [84], we estimated that the maximum $S(t)$ in Fig. (6.2a) for pure LC corresponds to $Q_{h} \sim 1 \times 10^{16} \mathrm{~cm}^{-3}$, or an order parameter of $Q_{h} / N_{h} \sim 4 \times 10^{-6}$.

We fitted quantitatively the observed $S(t)$ of pure LC in Fig. (6.2a) using Eqs. (6.1) and (3.27), with $Q_{h}^{\text {pure }}(t)$ given in Eq. (3.20). Using the nanosecond pump/probe setup we measured the longest exponential decay time $\tau_{1}$ of $Q_{h}^{\text {pure }}$ and found $\tau_{1}=16.5 \pm 0.8 \mathrm{~ns}$, which agrees with the result of Ref. [54]. From previous works [45, 46, 48, 49, 50, 54, 56, 57] we know that the various exponential decay times $\tau_{k}$ of $Q_{h}^{\text {pure }}$ differ in order of magnitude, so to find them we used a successive tail matching procedure. We started by fitting the long tail of $S(t)$ by the $k=1$ component alone in Eq. (3.20) with $\tau_{1}=16.5$ ns and $C_{1}$ as the adjustable parameter. The $k=1$ component was then subtracted out from the signal, and another tail-matching was performed to determine the $k=2$ component, with $\tau_{2}$ and $C_{2}$ as adjustable parameters, and similarly for higher $k$ 's. We found that we need to include up to $k=4$ to adequately describe the observed data. Figure 6.3 shows the $k$-components obtained from the fit, with: $\tau_{1}=16.5 \pm 0.8 \mathrm{~ns}, \tau_{2}=320 \pm 60 \mathrm{ps}, \tau_{3}=23.5 \pm 5.0 \mathrm{ps}, \tau_{4}<5$ ps, and $C_{1} / C_{2} / C_{3} / C_{4}=1 / 0.010 / 0.0041 / 0.0088$ with uncertainties of $5 \%, 10 \%, 20 \%, 12 \%$, respectively, and as seen in Fig. (6.2a), the overall fit with the experimental curve is very good. The peak of the response curve at $t \sim 0$ in Fig. 6.2a would have a detectable shift if $\tau_{4} \geq 5$ ps. The absolute magnitude of the signal from the pure sample was used as a scale factor for subsequent fitting of results from the dye-doped LC sample described below. 


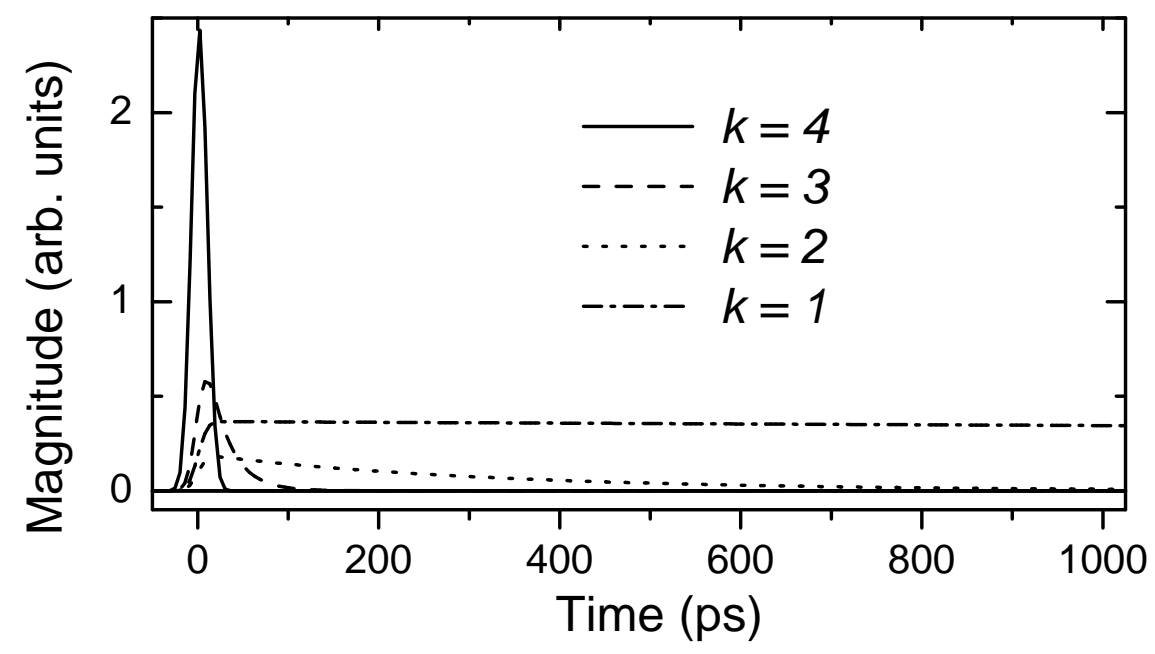

Figure 6.3: The four components of $\Delta \alpha_{h}^{\prime} Q_{h}^{\text {pure }}(t)$, described by Eq. (3.20), used to obtain the fit in Fig. 6.2a for the transient grating signal from pure LC. Vertical scale is the same as in Fig. 6.2.

Turning our attention now to the signals from the dye-doped sample, shown in Figs. $6.2 \mathrm{~b}-\mathrm{d}$, we see that $S(t)$ at the three $\lambda_{\text {probe }}$ s all roughly follow the same qualitatively behavior: a sharp peak near $t=0$, similar to that of the pure signal, then a decrease through a minimum, and then a gradual increase to about the same magnitude for all three $\lambda_{\text {probe }}$ at $t \simeq 1 \mathrm{nsec}$. The differences appear in the detailed behavior of $S(t)$ for $t \leq 700 \mathrm{ps}$ in magnitudes and positions of the minimum. They originate from significant contribution to $S(t)$ from the dye and from the dependence of $\Delta \alpha_{g}$ and $\Delta \alpha_{e}$ on $\lambda_{\text {probe. }}$ As the dye contribution to $S(t)$ decays away for $t \geq 700$ (recall that $\tau_{Q e}=490 \mathrm{ps}$ and $\tau_{D g}=170 \mathrm{ps}$ ), the signal $S(t)$ becomes dominated by the LC host contribution, which is insensitive to $\lambda_{\text {probe }}$. Note that at $t \simeq 1 \mathrm{~ns}, S(t)$ for the dye-doped LC is higher than that of the pure sample; this signifies the enhanced reorientation effect in the dye-doped LC case. 
To understand quantitatively the signal from the dye-doped sample, we first note that at the chosen near-IR probe wavelengths, $\Delta \alpha_{g}^{\prime \prime}=\Delta \alpha_{h}^{\prime \prime}=0$, and $\Delta \alpha_{e}^{\prime \prime}$ is small but nonvanishing. (Recall that we have characterized the relative magnitudes of $\Delta \alpha_{e}^{\prime \prime}$ at these probe wavelengths in the transient dichroism experiment of the previous chapter.) Knowing that $\left[Q_{i}^{x x}(t)-Q_{i}^{y y}(t)\right] \propto Q_{i}(t)$, we have from Eqs. (3.27) and (6.1)

$$
S(t) \propto\left[\Delta \alpha_{e}^{\prime} Q_{e}+\Delta \alpha_{g}^{\prime} Q_{g}+\Delta \alpha_{h}^{\prime} Q_{h}^{\text {pure }}+\Delta \alpha_{h}^{\prime} Q_{h}^{e n h}\right]^{2}+\left[\Delta \alpha_{e}^{\prime \prime} Q_{e}\right]^{2}
$$

where $Q_{e}(t)$ and $Q_{g}(t)$ are given by Eqs. (3.8) and $Q_{h}^{\text {pure }}(t)$ and $Q_{h}^{e n h}(t)$ by Eq. (3.20). Using the amplitude of the $k=1$ component of $Q_{h}^{\text {pure }}$ as the normalization constant, we can use Eq. (6.2) to fit the experimental curves, taking $\Delta \alpha_{e}^{\prime} /\left(\Delta \alpha_{h}^{\prime} C_{1}\right), \Delta \alpha_{g}^{\prime} /\left(\Delta \alpha_{h}^{\prime} C_{1}\right)$, $\Delta \alpha_{e}^{\prime \prime} /\left(\Delta \alpha_{h}^{\prime} C_{1}\right), u_{h g}$, and $u_{h e}$ as fitting parameters. The last two parameters appear in the expression of $Q_{h}^{e n h}(t)$ in Eq. (3.20). Furthermore, we found that it is advantageous to replace $u_{h g}$ and $u_{h e}$ with two dimensionless parameters: $r_{u} \equiv u_{h g} / u_{h e}$ and $\eta_{\infty}$ (Eq. (3.25)), which represent the ratio and (scaled) difference, respectively, of the mean-field interaction energies. It is known that $u_{h g}>0$ from ground-state dichroism measurement of nematic-phase dye-doped LC samples [13], and we expect $u_{h g} \leq u_{h e}$ from physical arguments discussed in section 2.2. Thus the parameter $r_{u}$ is restricted to the range $0<r_{u} \leq 1$.

The values of $\Delta \alpha_{e}^{\prime}$ and $\Delta \alpha_{g}^{\prime}$ are expected to be of opposite sign to $\Delta \alpha_{h}^{\prime}$. This is because the easy-polarizing axis for $1 \mathrm{AAQ}$ is along the $\mathrm{C}=0$ bonds, perpendicular to the molecular long axis. The presence of terms with opposite signs in the first square brackets of the right hand side of Eq. (6.2) means that as time proceeds, $S(t)$ could go through a minimum if the birefringence terms in the first square brackets cancel each other, and 
the minimum would be $\left[\Delta \alpha_{e}^{\prime \prime} Q_{e}\right]^{2}$ at that particular time. Indeed the observed $\sqrt{S(t)}$ for each probe wavelength, described in Fig. $6.2 \mathrm{~b}-\mathrm{d}$, does go through a minimum at around 200-400 ps. The corresponding dichroism magnitude at minimum $\sqrt{S(t)}$ yields the relative magnitudes of the dye excited-state dichroism at the different probe wavelengths: $\Delta \alpha_{e}^{\prime \prime}(925 \mathrm{~nm}) / \Delta \alpha_{e}^{\prime \prime}(848 \mathrm{~nm})=0.50, \Delta \alpha_{e}^{\prime \prime}(1024 \mathrm{~nm}) / \Delta \alpha_{e}^{\prime \prime}(848 \mathrm{~nm})=0.0$, with uncertainty at $12 \%$. These values agree within experimental error with what we found in the transient dichroism measurement described in the previous chapter.

Figure 6.4a shows the excited and ground-state dye anisotropies $Q_{e}(t)$ and $Q_{g}(t)$, as given by Eqs. (3.8) with the various $\tau$ 's experimentally determined previously. Note the negative sign on $Q_{g}(t)$. From $Q_{e}(t)$ and $Q_{g}(t)$ we can calculate the transient torque that reorients the LC host, as represented by the quantity $Q_{e}(t)+r_{u} Q_{g}(t)$ (refer to Eq. (3.20)). The transient torque for different values of $r_{u}$ is shown in Fig. 6.4b. Representative curves for $Q_{h}^{e n h}(t)$, as a result of the dye excitation (Eq. (3.20)), with different values of $\eta_{\infty}$ and $r_{u}$, are shown in Fig. 6.4c. We see that $\eta_{\infty}$, being the steady-state enhancement factor, determines the asymptotic value of $Q_{h}^{e n h}(t)$ as $t$ becomes much larger than $\tau_{D g}$ and $\tau_{Q e}$ $(t \geq 800 \mathrm{ps})$. As seen in Fig. 6.5a, among the various terms described by Eq. (6.2) to compose $S(t), \Delta \alpha_{h}^{\prime} Q_{h}^{e n h}$ is dominant in the time regime $t \geq 800 \mathrm{ps,}$ and thus it limits the range of possible values for $\eta_{\infty}$. The best-fit value was $\eta_{\infty}=3.3 \pm 0.4 / 0.2$.

The situation is different in determining $r_{u}$. Different values of $r_{u}$ affect the net torque of dye on host molecules $\left(\propto Q_{e}(t)+r_{u} Q_{g}(t)\right)$ (Fig. 6.4b) mainly in the early time $t \leq 400$ ps because $Q_{g}(t)$ decays much faster than $Q_{e}(t)$ (Fig. 6.4a). The subsequent effect of different $r_{u}$ on $Q_{h}^{e n h}(t)$ is moderate and also mainly in the early time $t \leq 600 \mathrm{ps.} \mathrm{In} \mathrm{fitting}$ 


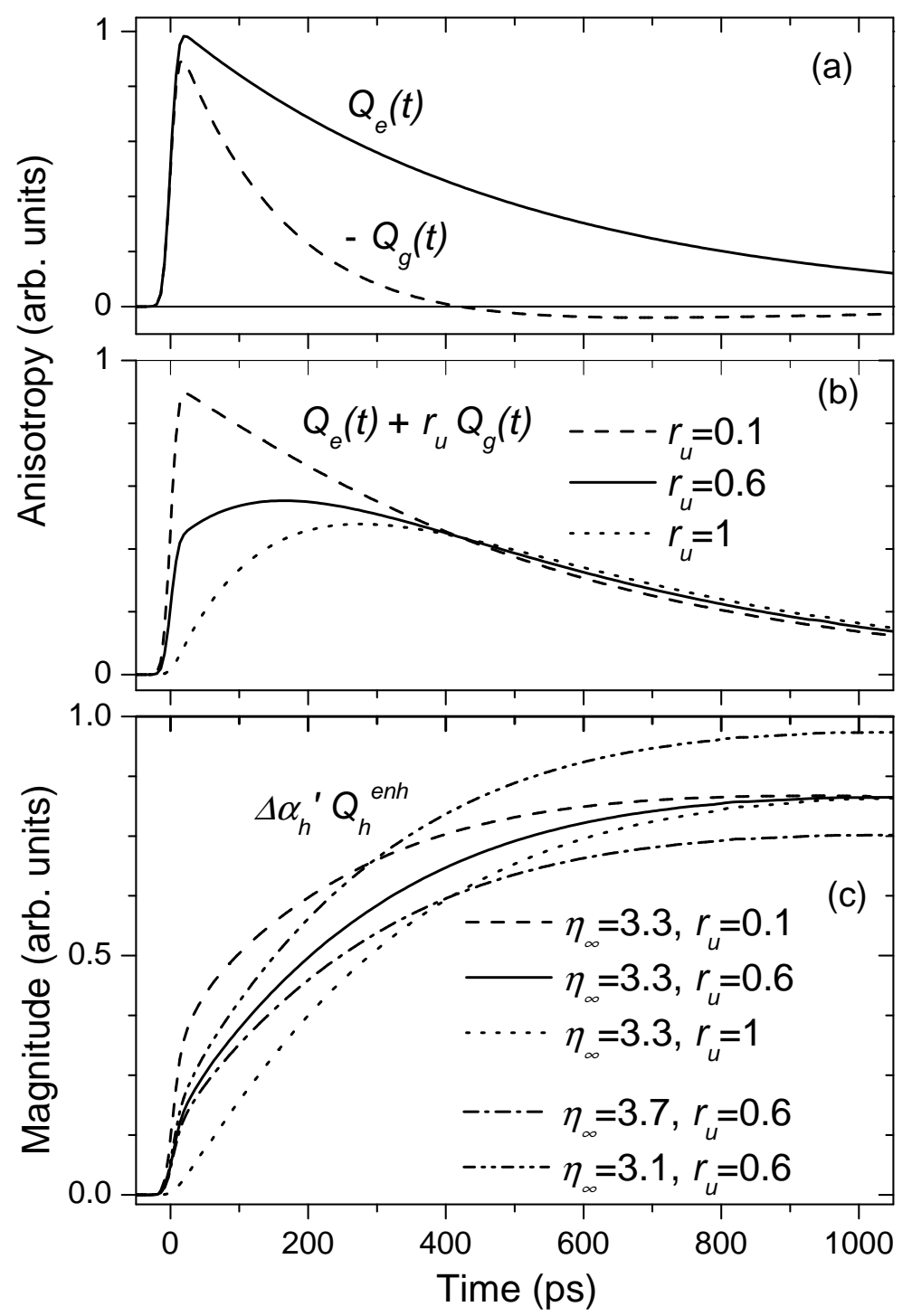

Figure 6.4: Fitting of the dye torque: (a) Excited and ground state dye anisotropies. Note the negative sign on $Q_{g}(t)$. (b) Net transient torque from the dye population acting on the LC host, represented by $Q_{e}(t)+r_{u} Q_{g}(t)$, for different values of $r_{u}$. (c) LC anisotropy created by the dye torque, $Q_{h}^{e n h}(t)$, for different values of $\eta_{\infty}$ and $r_{u}$. Vertical scale of (c) is the same as in Fig. 6.2. 


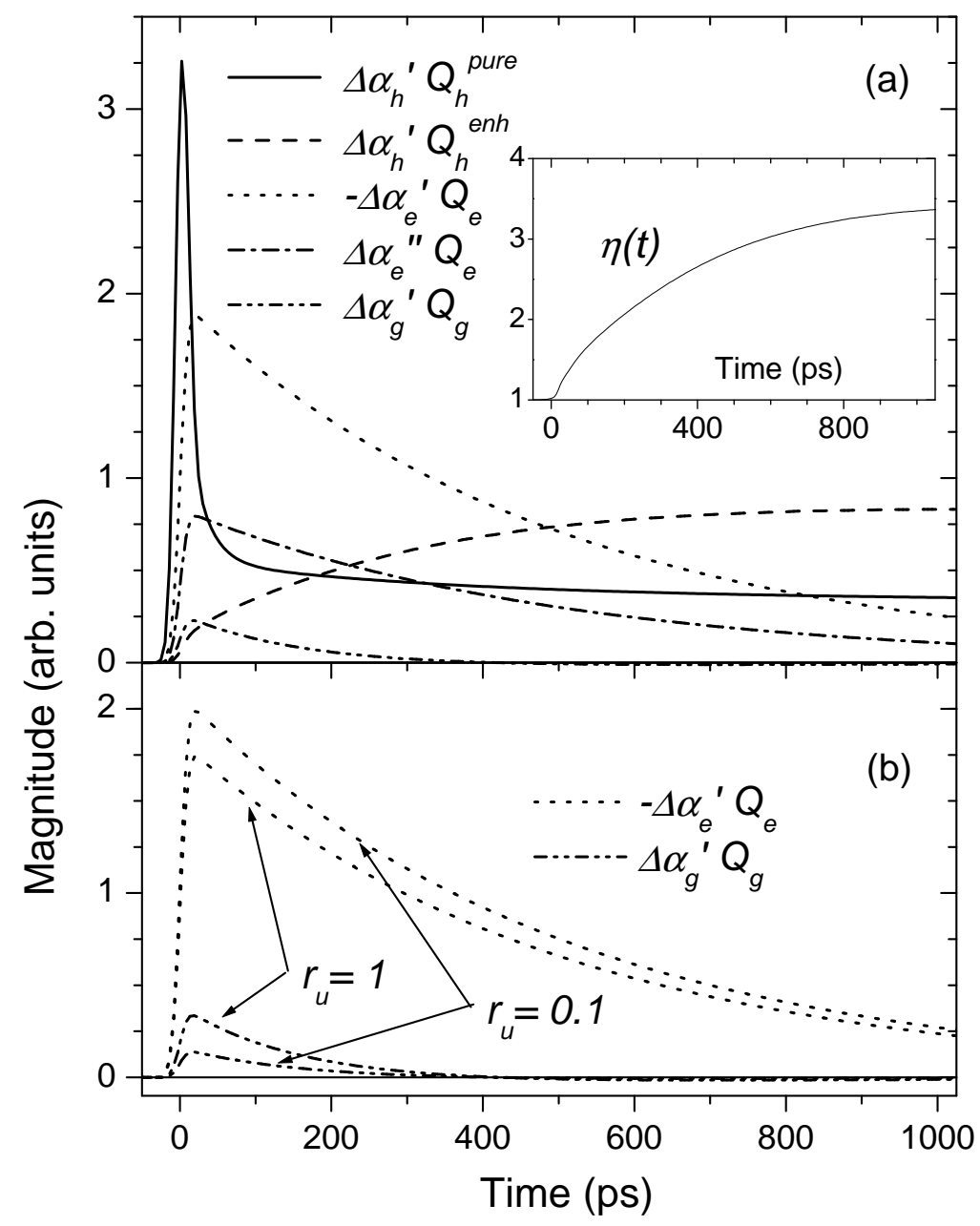

Figure 6.5: Fitting of the transient grating signal from dye-doped LC: (a) The five terms (Eq. (32)) used to obtain the fit in Fig. 6.2b, where $\eta_{\infty}=3.3$ and $r_{u}=0.6$. Note the negative sign on $\Delta \alpha_{e}^{\prime} Q_{e}$. Inset of (a) gives the corresponding transient enhancement factor $\eta(t)$. (b) For different values of $r_{u}$, with the dye contribution to the signal adjusted as shown, the fit remains practically the same as in Fig. 6.2b. Vertical scale is the same as in Fig. 6.2. 
of the measured signal $S(t)$ through Eq. (6.2), it turns out that the change of $Q_{h}^{e n h}(t)$ due to different $r_{u}$ could be offset by adjusting the values of the $\Delta \alpha_{e}^{\prime}$ and $\Delta \alpha_{g}^{\prime}$ to change the dye contribution, $\left[\Delta \alpha_{e}^{\prime} Q_{e}(t)+\Delta \alpha_{e}^{\prime} Q_{g}(t)\right]$, in Eq. (6.2). We found that any value of $r_{u}$ within the range $0<r_{u} \leq 1$ would yield a satisfactory fit. This is shown in Fig. $6.5 \mathrm{~b}$, for $r_{u}=0.1$ and 1 .

As seen in Figs. 6.2b-d, we obtained remarkably good fits between theory and experiment at all three probe wavelengths. The various terms of the fit, described in Eq. (6.2), for the case of $\lambda_{\text {probe }}=848 \mathrm{~nm}$ (Fig. 6.2b) are shown in Fig. 6.5a. The relative magnitudes of the dye polarizability anisotropies used to obtain the fits for the signal at all three probe wavelengths (Figs. 6.2b-d) are listed in Table 6.1. At these wavelengths, $\Delta \alpha_{e}^{\prime}$ is significantly larger than the corresponding $\Delta \alpha_{g}^{\prime}$ because it is near or at resonance, as demonstrated by the nonzero values of $\Delta \alpha_{e}^{\prime \prime}$. By comparing with the signal from benzene as mentioned earlier, the value of $\Delta \alpha_{g}^{\prime}$ at $848 \mathrm{~nm}$ used in the fit of Fig. $6.2 \mathrm{~b}$ is estimated to be $\simeq 2 \AA^{3}$, which is comparable to the values obtained for similar compounds [85]. The estimated value of $\Delta \alpha_{e}^{\prime \prime}$ at $848 \mathrm{~nm}$ corresponds to $\Delta \sigma_{e} \simeq 4.2 \times 10^{-18} \mathrm{~cm}^{2}$ (for comparison, $\Delta \sigma_{g}=1.5 \times 10^{-17} \mathrm{~cm}^{2}$ at $\left.532 \mathrm{~nm}\right)$.

From the fitting result of $\eta_{\infty}$ and $r_{u}$, together with the material parameters of the $1 \mathrm{AAQ} / 5 \mathrm{CB}$ system, we can calculate $u_{h e}$ and $u_{h g}$. At $\lambda_{\text {pump }}=532 \mathrm{~nm}$, the linear absorption of the dye-doped sample is $\beta=6.8 \mathrm{~cm}^{-1}, n=1.58, \Delta \alpha_{h}^{\prime}=17.6 \AA^{3}$ [38], yielding $A / B=4.65 \times 10^{10} \mathrm{eV}^{-1} \cdot \mathrm{s}^{-1}$. Then the result of $\eta_{\infty}=3.3 \pm 0.4 / 0.2$ and $0<r_{u} \leq 1$ yields the following approximate range for the mean-field orientational energies: $0.090 \leq u_{h e} \leq 0.17 \mathrm{eV}$ and $0<u_{h g} \leq 0.17 \mathrm{eV}$. These are comparable to the values measured and deduced in earlier 


\begin{tabular}{rccc}
\hline \hline & $\Delta \alpha_{g}^{\prime}$ & $\Delta \alpha_{e}^{\prime}$ & $\Delta \alpha_{e}^{\prime \prime}$ \\
\hline$\lambda_{\text {probe }}=848 \mathrm{~nm}$ & 1 & 7.4 & 3.2 \\
$925 \mathrm{~nm}$ & 1.0 & 5.5 & 1.6 \\
$1024 \mathrm{~nm}$ & 1.0 & 5.5 & 0.0 \\
\hline \hline
\end{tabular}

Table 6.1: Relative magnitudes of dye polarizabiblity anisotropies from the transient grating experiment, from the fits in Fig. 6.2b-d. $\Delta \alpha_{g}^{\prime}$ at $\lambda_{\text {probe }}=848 \mathrm{~nm}$ is estimated to be $\simeq 2 \AA^{3}$. studies of the Jánossy effect in similar anthraquinone-dye-doped LC systems [13, 22].

In the next section we discuss the relevance of our experimental results. 


\section{Chapter 7}

\section{Discussion of the reorientational}

\section{dynamics of dye-doped liquid}

\section{crystals}

Theoretical fitting of the experiment described in the previous chapter allowed us to deduce the time-dependent reorientation enhancement $\eta(t)$ (Eqs. (3.20-21)). As shown in the inset of Fig. 6.5a, for a representative case of $\eta_{\infty}=3.3$ and $r_{u}=0.6, \eta(t)$ behaves as we expect: it starts at 1 at $t=0$, increases with time, corresponding to when the net extra torque resulting from $Q_{e}(t)$ and $Q_{g}(t)$ builds up, and asymptotically approaches the steady-state value as $Q_{e}(t)$ and $Q_{g}(t)$ decay away. The value $\eta_{\infty}=3.3 \pm 0.4 / 0.2$ we deduced from our transient measurements agrees with the value $\eta_{\infty}=3.0 \pm 0.3$ that we obtained using the quasi-steady-state, nanosecond setup (Chapter 4). Thus we have elucidated the 
transition of the enhancement process from the transient regime to the quasi-steady-state regime.

The enhancement effect relies on two key features. Referring to the expression for $\eta_{\infty}$ in Eq. (3.25), it first requires $\left(A u_{h e} \tau_{Q e}\right) / B \geq 1$. The parameter $A$ quantities the absorption strength of the dye, $u_{h e}$ quantifies the orientational coupling between LC molecules and excited dye molecules, and $\tau_{Q e}$ quantifies the time window that the excited dye molecules exert a torque on the host, while $B$ quantifies the direct optical torque. However, having a strong dye torque is not enough - it also requires the overall reorientational effects of dye molecules in the excited and ground states on the host are different, represented by $\left(u_{h g} / u_{h e}\right)\left(\tau_{D g} / \tau_{D e}\right) \neq 1$. These two features combine to yield an $\eta_{\infty}$ different from 1.

For the $1 \mathrm{AAQ} / 5 \mathrm{CB}$ system, we have found that the ground-state dye anisotropy decays away significantly faster than the excited-state counterpart, $\tau_{D g} / \tau_{D e}=0.2$, so that the net dye-assisted reorientation of the LC host at later times is mainly due to the excited dye molecules, with less than $20 \%\left(=r_{u}\left(\tau_{D g} / \tau_{D e}\right)\right)$ counterbalanced by the negative action of the ground-state dye anisotropy. The contribution to the enhanced reorientation of the LC host from the change in rotational diffusion between the dye excited and ground states is so large that it masks out the contribution, if any, from the change in mean-field reorientational energies.

The observed decrease in rotational diffusion upon excitation of the dye in $5 \mathrm{CB}$ is the largest reported in the literature so far $[63,75,76,77,78,79,80]$, but we note that our study is the first to directly probe the ground-state rotational dynamics of a dye [26]. As mentioned earlier, the first excited state of $1 \mathrm{AAQ}$ corresponds to an intra- 


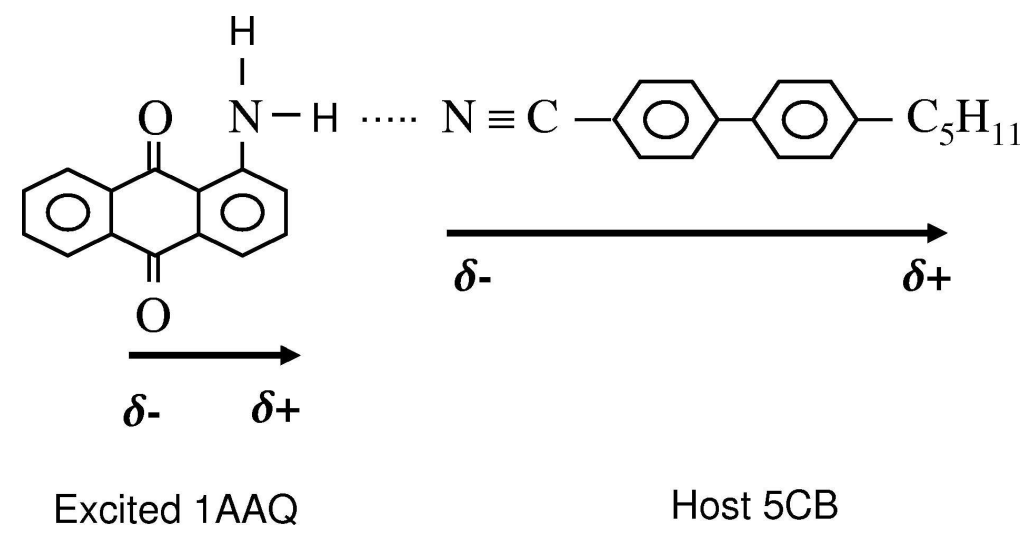

Figure 7.1: Interaction between excited $1 \mathrm{AAQ}$ and $5 \mathrm{CB}$ that could enhance intermolecular friction upon excitation of $1 \mathrm{AAQ}$. Solid arrows indicate the dipole moments. Dotted line indicates the hydrogen-bond between the molecules.

molecular charge-transfer state where electronic charge is transferred from the amino group to the carbonyl groups [35], making the amino group more prone to form a hydrogen bond with available hydrogen-bonding moieties of the surrounding solvent molecules such as the cyano group of $5 \mathrm{CB}[14,86]$ (Fig. 7.1). Other possible modes of interaction between $1 \mathrm{AAQ}$ and $5 \mathrm{CB}$, such as dipole-dipole interaction or van der Waals, are also expected to increase in strength upon excitation of $1 \mathrm{AAQ}$ due to the dipolar characteristic and the more extended electronic cloud distribution of the excited state. This overall stronger interaction leads to an increase of frictional forces between the excited $1 \mathrm{AAQ}$ and neighboring $5 \mathrm{CB}$ molecules, impeding rotational diffusion of the excited molecules as compared with their ground-state counterpart. It has been shown before that large change in dye rotational diffusion could result from relative small change in the overall guest-host interaction energy [13, 87]. According to Ref. [87], in the simplest approximation, the rotational diffusion 
constant depends on the guest-host interaction through the Arrhenius-type relation

$$
D_{g, e} \propto e^{-E / k T}
$$

where $E$ is the associated activation energy. For the temperature and material system used in this work, $E \sim 10 k_{B} T$ [13]; a change of $\sim 15 \%$ in $E$ is enough to change $D_{g, e}$ by a factor of 5 .

In both Ref. [13] and our study, the values of $u_{h g}$ and $u_{h e}$ are $\sim 0.2 \mathrm{eV}$, compared to the typical value $u_{h h} \simeq 0.4 \mathrm{eV}$ for the mean-field interaction of the host LC. They are consistent with the typical strength known for polar dipole-dipole and hydrogen-bonding interactions [39].

We have found good agreement between model prediction and experimental data in our time-resolved study of the Jánossy effect, thus significantly expanding our confidence in the model. The same physical underpinnings of the model apply to dye-doped LC in the nematic phase $[17,18,19]$. It has been suggested that a change in rotational diffusion upon photo-excitation, as observed in our study, could also be the driving force in the optical reorientation of azo-dye systems, working either in conjunction with or, in certain cases, instead of the well-known photo-isomerization of the azo dyes [63]. In fact, several azo dye-doped LC systems have been found to exhibit enhanced optical reorientation $[11,65]$. An investigation of the azo-dye/LC system following the approaches of our study, with emphasis on characterizing separately the dynamical responses of the various molecular species present after photo-excitation, could be readily carried out to answer the question whether the same physical mechanism, as elucidated in the present study, is at work in 
azo-dye/LC systems.

In terms of methodology, we would like to highlight that through judicious use of variable wavelengths and pulse sequences in the standard pump/probe technique, we have been able to characterize separately the dynamical responses of excited-state dye, groundstate dye, and host molecules, allowing us to directly follow the response of the host to the dye reorienting torque. This approach of looking at the host response is similar in spirit to recent studies of solvation dynamics in Refs. [31, 32, 33]. Our study, with picosecond excitation pulses, focuses exclusively on the diffusional reorientational dynamics, while the other studies, with femtosecond excitation pulses, could probe the faster diffusion, libration, and interaction-induced responses [33]. The latter also found significant enhancement of the solvent responses upon electronic excitation of the guest molecules.

The success of the mean-field model in describing the guest-host interaction in isotropic dye-doped LC could yield insights into the nature of the LC host itself. Recall that in the isotropic phase the LC molecules still possess short-range orientational correlation, manifested as pseudo-nematic domains of finite size existing within the macroscopically isotropic material [34]. At the doping concentration that we used, we always have much less than one dye molecules per pseudo-nematic domains. (This can be seen by recalling that the size the domains is characterized by a correlation length $\xi$ given by: $\xi=\xi_{o}\left[T^{*} /(T-\right.$ $\left.\left.T^{*}\right)\right]^{1 / 2}$, where for $5 \mathrm{CB}, \xi_{o} \simeq 0.5 \mathrm{~nm}$ and $T^{*}=34.2^{\circ} \mathrm{C}$. So for $T=50^{\circ} \mathrm{C}$ and $T=T_{n i}=$ $35.3^{\circ} \mathrm{C}$, we have $\xi \simeq 1.3$ and $8.8 \mathrm{~nm}$, corresponding to domains containing $\simeq 10$ and 500 LC molecules, respectively, while the ratio of dye to $\mathrm{LC}$ molecules is $\simeq 1: 2000$.) Thus due to the short-range nature of the dipolar interaction responsible for the guest- 
host orientational coupling [39], the reorientation of LC by dye molecules is necessarily an intra-domain process. The dye molecules, under the mean-field approximation of the guesthost interaction, are expected to couple to all modes of orientational relaxation, $(k=1$ through 4 in Eq. (3.20)), of the LC host. While the $k=1$ component corresponds to collective response of LC following mean-field host-host coupling, components with $k \geq$ 2 come from increasingly more local responses beyond the mean-field description of the host-host interaction [47]. More careful investigation of the reorientation enhancement by dye excitation in the short time regime could reveal how guest-host interaction affects reorientation of host molecules on different length scales [59, 60].

An interesting prospect for future work is to look for the Jánossy effect in chiral liquid crystalline media, such as those discussed briefly in section 2.1.2. It is known that chiral dopants could induce or modify the chiral structure of nematic LC's through guesthost interaction $[3,88,89,90]$. A change in the chiral guest-host interaction by, for example, photo-excitation of the guest chiral molecules, would lead to a change in the macroscopic chirality of the system, as have been observed with chiral dopants that exhibits photoisomerization, providing new prospects for optoelectronic applications [91, 92]. Photoexcited non-conformational chirality change of the chiral guest molecules could also induce a significant change in the chiral structure of the host. The chiral aminoanthraquinone dyes discussed in Ref. [93] seem particularly suited for a search for the chiral Jánossy effect.

In the next chapter we present preliminary results on one extension of the work presented so far. It is the search for the Jánossy effect in a pure liquid crystalline medium. 


\section{Chapter 8}

\section{Excitation-enhanced optical}

\section{reorientation in pure liquid crystals}

\subsection{Introduction}

An important prediction of the model of the Jánossy effect described in the previous chapters is that the same effect could exist in a pure absorbing material. In this case, the system is homogeneous initially, and absorption of the polarized light introduces the "guest" population: the oriented excited molecules and the orientational holes in the ground state distribution. These will then compete in reorienting the ground-state molecules, just as excited dye molecules and the ground-state dye orientational holes act on the liquid crystal molecules in the dye-doped case. In this pure material, a net anisotropy will be created by the absorption if either (i) the rotational diffusion time of the molecules is different in the excited state than in the ground state, or (ii) the orientational intermolecular coupling 
between excited and ground-state molecules is different than that between ground-state and ground-state molecules.

Confirmation of this prediction of the Jánossy effect in a pure absorbing material clearly would further enhance our confidence in the model. From an application standpoint, the Jánossy effect in pure liquid crystals would present a new and simple way to enhance the already large optical nonlinearity of this material. Thus we set out to look for the Jánossy effect in pure liquid crystals in the isotropic phase, using similar methodology as we used in the case of dye-doped liquid crystal case. We describe our preliminary results in this chapter.

\subsection{Theory}

The theoretical formalism governing the rotational dynamics of pure isotropic liquid crystals subjected to photoexcitation is similar to those used to describe the dye-doped liquid crystals of previous chapters. The only difference is that now we don't have separate $g$ (ground-state guest) and $h$ (ground-state host) populations. There are now just two molecular species, $i=g$ and $e$, corresponding to liquid crystals in the ground and excited states, respectively. The same derivation as in the dye-doped case could be followed to arrive at the equations of motion for the anisotropies $Q_{g}(t)$ and $Q_{e}(t)$.

Looking back at case of dye-doped liquid crystals studied in the previous chapters, we see that the faster relaxation channels of the liquid crystal response, terms with $k>1$

in Eqs. (3.20) (where $\tau_{k} \leq$ few hundreds ps), are necessary to describe the subnanosecond 
dynamics of the system, where the dye responses are relevant (recall that $\tau_{D g}=170 \mathrm{ps}$ and $\tau_{Q e}=490 \mathrm{ps}$ for the dye.) For the long time or steady-state regime (ns and above), the response of system is adequately described by the $k=1$ component alone, with $\tau_{1} \geq$ tens of nanoseconds. In the present case of pure liquid crystal, it turns out that all the relevant time constants, i.e. $\tau_{D_{e}}, \tau_{f}, \tau_{D_{g}}$, are $\sim 1$ ns. Then, to simplify the picture, we could include only the $k=1$ component in the description of the liquid crystal dynamics. Hence we need not go beyond the mean-field approximation of the liquid crystal-liquid crystal interaction (as was done in sections 3.2.2-3 to arrive at the faster relaxation channels of $k>1$ ), since the $k>1$ components are beyond the mean-field model.

Thus in this chapter we will describe the pure liquid crystals under the meanfield approximation for both the excited state - ground state interaction and ground state - ground state interaction. In Appendix A we describe in details the derivation of the dynamical equations under the mean-field approximation for all intermolecular interactions, both for the dye-doped and the pure liquid crystals case. From section A.3 we get the following dynamical equations:

$$
\begin{aligned}
\frac{\partial Q_{e}}{\partial t}+\frac{Q_{e}}{\tau_{D_{e}}} & =+A I(t)-\frac{Q_{e}}{\tau_{f}} \\
\frac{\partial Q_{g}}{\partial t}+\frac{Q_{g}}{\tau_{D_{g}}} & =-A I(t)+\frac{Q_{e}}{\tau_{f}}+\frac{u_{g g} N_{g}}{15 k_{B} T \tau_{D_{g}} N_{t}} Q_{g}+\frac{u_{g e} N_{g}}{15 k_{B} T \tau_{D_{g}} N_{t}} Q_{e}
\end{aligned}
$$

The first two terms on the right hand side (RHS) of Eqs. (8.1-2) describe the population transfer due to optical excitation and spontaneous depopulation, respectively. The third and fourth terms on the RHS of Eq. (8.2) describe the interaction between ground-state molecules and between ground-state and excited-state molecules, respectively. The non- 
resonant direct optical torque has been neglected since its effect in generating population anisotropy is quite smaller than that of the resonant absorption, as demonstrated in Appendix A. The effect of intermolecular interaction on the excited state anisotropy is also negligible, as explained in Appendix A, and thus not included in Eq. (8.1).

From Eqs. (8.1-2) we see that after the picosecond excitation pulse is over, $Q_{e}$ is positive, representing the anisotropy in the excited state parallel to the excitation polarization direction, while $Q_{g}$ is negative, representing the perpendicularly-oriented orientational holes in the ground state. $Q_{e}$ and $Q_{g}$ would then compete to reorient the rest of the ground-state molecules, towards the parallel and perpendicular direction, respectively. A net anisotropy would be created if one effect wins over the other.

Equations (8.1-2) could be rewritten as:

$$
\begin{aligned}
\frac{\partial Q_{e}}{\partial t}+\frac{Q_{e}}{\tau_{Q_{e}}} & =+A I(t) \\
\frac{\partial Q_{g}}{\partial t}+\frac{Q_{g}}{\tau_{g}} & =-A I(t)+\left(\frac{T^{*} u_{g e}}{T \tau_{D_{g}} u_{g g}}+\tau_{f}^{-1}\right) Q_{e}, \\
\text { where } \quad \tau_{Q e} & \equiv\left(1 / \tau_{D_{e}}+1 / \tau_{f}\right)^{-1}, \tau_{g} \equiv \tau_{D_{g}}\left(\frac{T}{T-T^{*}}\right), T^{*} \equiv \frac{u_{g g}}{15 k_{B}} .
\end{aligned}
$$

$\tau_{g}$, equivalent to $\tau_{1}$ in section 3.2 .2 , is the collective rotational relaxation time that exhibits pretransitional behavior due to intermolecular interaction. Knowing that all the relevant time constants in Eqs. (8.3-4) are of the order 1 ns or above, much longer than the excitation pulse width of $\sim 15$ ps, Eqs. (8.3-4) have the approximate solutions

$$
\begin{aligned}
& Q_{e}(t) \simeq+Q_{o} e^{-t / \tau_{Q_{e}}} \\
& Q_{g}(t) \simeq-R Q_{o} e^{-t / \tau_{Q_{e}}}+(R-1) Q_{o} e^{-t / \tau_{g}}
\end{aligned}
$$


where $Q_{o} \equiv A \int_{-\infty}^{\infty} I(t) d t, t=0^{+}$is taken as the time right after the pump pulse is essentially over, and $R$ is given by:

$$
R \equiv\left[\left(\frac{T^{*}}{T}\right)\left(\frac{u_{g e}}{u_{g g}}\right)+\left(\frac{\tau_{D_{g}}}{\tau_{f}}\right)\right] \cdot\left[\left(\frac{T^{*}}{T}\right)+\left(\frac{\tau_{D_{g}}}{\tau_{f}}\right)+\left(\frac{\tau_{D_{g}}}{\tau_{D_{e}}}\right)-1\right]^{-1}
$$

If the system is kept relatively close to the nematic-transition temperature, we can make the approximation $T^{*} / T \simeq 1$, then:

$$
R \simeq\left(\frac{1}{r_{u}}+r_{f}\right) \cdot\left(\frac{1}{r_{\tau}+r_{f}}\right)
$$

where $r_{u} \equiv u_{g g} / u_{g e}, r_{\tau} \equiv \tau_{D_{g}} / \tau_{D_{e}}$, and $r_{f} \equiv \tau_{D_{g}} / \tau_{f}$.

From Eqs. (8.5-6), we see that after excitation the excited-state anisotropy decays single exponentially with decay time $\tau_{Q_{e}}$. The ground-state anisotropy, on the other hand, follows a bi-exponential decay. It has one component that follows the same exponential decay as the excited state - this component can be viewed as the complementary image of the anisotropy in the excited state. The second component of ground state anisotropy has the decay time $\tau_{g}$, which is intrinsic to the rotational dynamics of the ground state (we expect $\tau_{Q_{e}} \neq \tau_{g}$ ). This component comes from the state-dependence of the intermolecular interaction, with the parameter $R$ gauging its magnitude.

If $r_{u}=r_{\tau}=1$, i.e. the excited-state and the ground-state molecules are exactly the same in term of their intermolecular interactions with neighboring molecules, then from Eq. (8.7) we have $R=1$, which then, following Eqs. (8.5-6), leads to the vanishing of the second component of $Q_{g}$ and a total anisotropy $Q_{e}+Q_{g}$ of zero. Physically this means that after the excitation pulse creates opposite anisotropies $Q_{e}$ and $Q_{g}$ in the excited state and ground state population, respectively, their decay and reorientational effect on the rest of 
the molecules are the same, hence the two anisotropies remain exactly complementary for all time, and thus no net molecular orientational anisotropy is produced. If there is a statedependence on the intermolecular interaction, we expect $r_{u} \neq 1$ and/or $r_{\tau} \neq 1$, leading to $R \neq 1$. Then, as can be seen from Eqs. (8.5-6), the second component of $Q_{g}$ is present and thus a difference in magnitude will develop between $Q_{e}$ and $Q_{g}$ as time evolves, leading to a net molecular anisotropy in the system. If the excited-state/ground-state interaction is stronger than the ground-state/ground-state interaction then $R$ is greater than 1 , and $R$ is less than 1 for the opposite case.

In the next section we will discuss the experimental strategy to observe the rotational dynamics as described by Eqs. (8.5-6), to determine whether $R \neq 1$.

\subsection{Experimental strategy and setup}

To observe the rotational dynamics of the photoexcited liquid crystal we use the same heterodyned pump/probe experimental setup as in the study of the dye rotational dynamics in Chapter 5. The schematic of the setup is shown in Fig. (8.1). Pump beam P, with wavelength in the UV, excites the material, then beam $\operatorname{Pr}$, in the visible range, probes the rotational dynamics of the system as described by Eqs. (8.5-6). The one new thing with our present setup as compared to that in Chapter 5 is the use of a removable quarter-wave plate for the probe wavelength (QWP in Fig. 8.1). Its usage is described below.

With the quarter-wave plate removed out of the path of the probe beam, the situation is the same as in Chapter 5. With the polarization of $\operatorname{Pr}$ set at $\hat{x}+\hat{y}$ before 


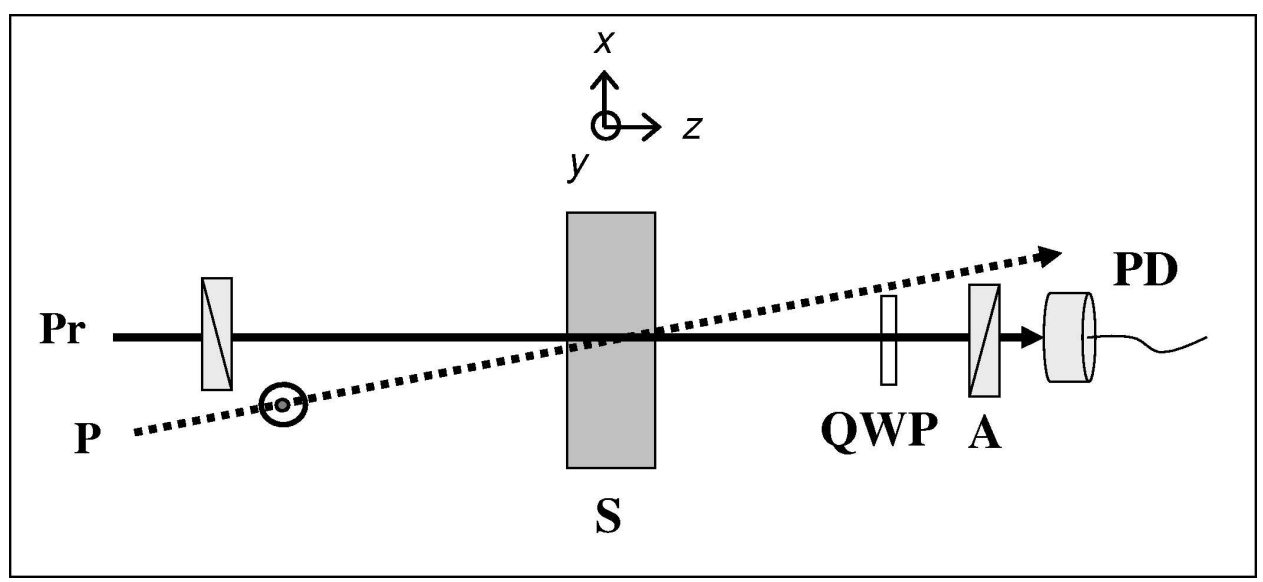

Figure 8.1: Schematic setup of the experiment. Pr: probe beam, P: pump beam, S: sample; QWP: removable quarter-wave plate for probe beam; A: analyzer polarizer; PD: photodetector.

the sample, and the analyzer A set at a small heterodyning angle $\gamma$ away from the crossed position of $-\hat{x}+\hat{y}$, then under the small-signal approximation the optical signal detected by the photodetector is given by:

$$
S_{t}(\gamma, t)=S_{o}\left\{\left[\frac{\pi l}{\lambda} \Delta n^{\prime \prime}(t)-\gamma\right]^{2}+\left[\frac{\pi l}{\lambda} \Delta n^{\prime}(t)\right]^{2}\right\}
$$

where $\Delta n^{\prime \prime}(t)$ and $\Delta n^{\prime}(t)$ represents the photoinduced dichroism and birefringence of the sample. As was done in Chapter 5, to focus on the time evolution of the induced dichroism, we measure the quantity:

$$
S(t) \equiv S_{t}(-\gamma, t)-S_{t}(\gamma, t) \propto \Delta n^{\prime \prime}(t)
$$

Now, if we insert the quarter-wave plate into the probe beam path, and align the fast axis along $\hat{x}+\hat{y}$, the role of the dichroism and birefringence is interchanged $[70,71]$. 
Specifically, analogous to Eq. (8.9) above, the optical signal in this case is:

$$
S_{t}^{\lambda / 4}(\gamma, t)=S_{o}\left\{\left[\frac{\pi l}{\lambda} \Delta n^{\prime}(t)-\gamma\right]^{2}+\left[\frac{\pi l}{\lambda} \Delta n^{\prime \prime}(t)\right]^{2}\right\}
$$

Similarly, to focus on the time evolution of the induced birefringence, we measure the quantity:

$$
S^{\lambda / 4}(t) \equiv S_{t}^{\lambda / 4}(-\gamma, t)-S_{t}^{\lambda / 4}(\gamma, t) \propto \Delta n^{\prime}(t)
$$

Now recall that the induced dichroism and birefringence is directly related to the population anisotropies of the sample (Eq. (3.26-27)), thus for our present case of photoexcited pure liquid crystals we have:

$$
\begin{aligned}
S(t) & \propto \Delta \alpha_{e}^{\prime \prime} Q_{e}(t)+\Delta \alpha_{g}^{\prime \prime} Q_{g}(t) \\
S^{\lambda / 4}(t) & \propto \Delta \alpha_{e}^{\prime} Q_{e}(t)+\Delta \alpha_{g}^{\prime} Q_{g}(t) .
\end{aligned}
$$

We see that we can measure independently the photoinduced dichroism and, with the help of the quarter-wave plate, birefringence of the sample. With the probe wavelength in the visible range, ground-state absorption is vanishing so $\Delta \alpha_{g}^{\prime \prime}=0$. Hence, substituting Eqs. (8.5-6) in for $Q_{e}(t)$ and $Q_{g}(t)$, Eqs. (8.13-14) can be written as:

$$
\begin{aligned}
S(t) & \propto e^{-t / \tau_{Q_{e}}} \\
S^{\lambda / 4}(t) & \propto\left(\Delta \alpha_{e}^{\prime} / \Delta \alpha_{g}^{\prime}-R\right) e^{-t / \tau_{Q_{e}}}+(R-1) e^{-t / \tau_{g}}
\end{aligned}
$$

Thus we see that if $R=1$ then $S(t)$ and $S^{\lambda / 4}(t)$ would follow the same single-exponential decay. The tell-tale sign that $R \neq 1$, and hence significant reorientational effect from the state-dependence intermolecular interaction, would be the observation of the decay 
component $\propto e^{-t / \tau_{g}}$ in the photoinduced birefringence of the sample, $S^{\lambda / 4}(t)$. Recall that $\tau_{g}$, which can be characterized independently, exhibits pretransitional behavior and can range from several to hundreds of nanoseconds depending on the temperature. $\tau_{Q_{e}}$, which can be measured through Eq. (8.15), does not exhibit pretransitional behavior, and is expected to be $\sim 1 \mathrm{~ns}$. Thus it would be quite straightforward to recognize in $S^{\lambda / 4}(t)$ the component $\propto e^{-t / \tau_{g}}$.

In our experiment, the laser system is the same as used previously in the dyedoped liquid crystal study. The UV pump beam is obtained by sum-frequency-generation of 1064-nm and visible pulses in a BBO crystal. Approximately, the pump and probe beams have energies of $5 \mu \mathrm{J}$ and $2 \mu \mathrm{J}$ per pulse, and beam diameters of $2.75 \mathrm{~mm}$ and $2.0 \mathrm{~mm}$, respectively. The wavelength of the probe beam is at $532 \mathrm{~nm}$, while the pump wavelength is set at $340 \mathrm{~nm}$, at the absorption edge of the liquid crystal 5CB. Practically all of the pump pulse is absorbed by the isotropic liquid crystal $\left(\mathrm{T}=50^{\circ} \mathrm{C}\right)$, which is housed in a $1 \mathrm{~mm}$-pathlength quartz cell, with transmission at $1 \%$.

\subsection{Result and Discussion}

We present the preliminary results of our experiment in Fig. (8.2). Shown are the time evolution of the measured photoinduced dichroism and birefringence of the liquid crystal sample, $S(t)$ and $S^{\lambda / 4}(t)$ of Eqs. (8.15-16), respectively. We see that the dichroism and birefringence follows very different time evolution, signifying that $R \neq 1$ and that the reorientational effect of the state-dependent intermolecular interaction is indeed substantial. 


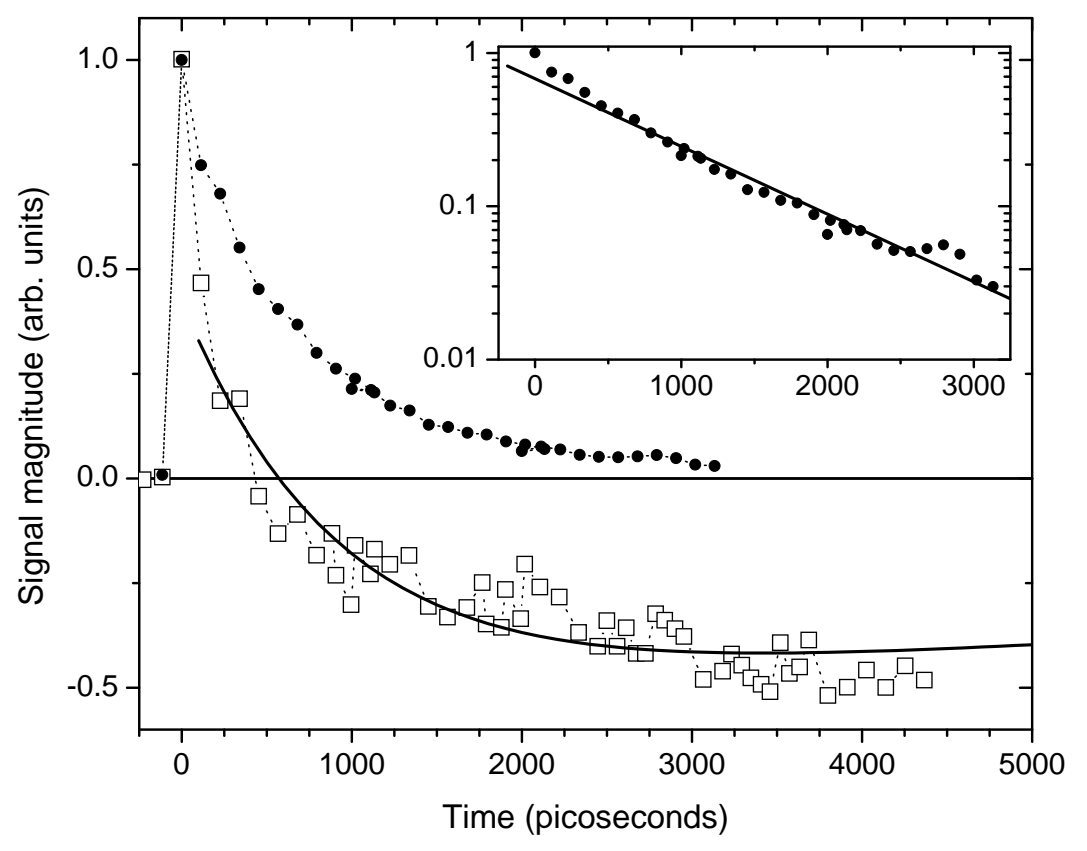

Figure 8.2: Time evolution of the dichroism $(\bullet)$ and birefringence $(\square)$ of the pure liquid crystal sample following photoexcitation at $t=0$. Dotted line is to guide the eye. Solid line in inset is single exponential fit of the dichroism data following Eq. (8.15), yielding $\tau_{Q_{e}}$. Solid line in main graph is bi-exponential fit of the birefringence data following Eq. (8.16).

The dichroism $S(t)$ follows a single exponential decay, as shown in the inset of Fig. (8.2), from which we deduced $\tau_{Q_{e}}=930 \pm 70 \mathrm{ps}$.

The birefringence $S^{\lambda / 4}(t)$ clearly does not follow a single exponential decay. It starts out being positive, then goes through zero, becomes negative, and seems to flatten out for $t \geq 3$ ns. Let's see what this means physically. First recall that a positive signal corresponds to overall positive anisotropy, which is defined as having molecular ordering parallel to the pump polarization. In Eq. (8.16), since excited-state absorption is present 
at $532 \mathrm{~nm}$ while ground-state absorption is not, we expect the excited-state polarizability is also larger than that of the ground state, and hence $\Delta \alpha_{e}^{\prime} / \Delta \alpha_{g}^{\prime}>1$. Also, we know that $\tau_{g}=16.5 \pm 0.8 \mathrm{~ns}$ (Chapters 6$)$. Thus the observed time evolution of $S^{\lambda / 4}(t)$ is consistent with the situation where $R$ is less than 1 , with the coefficient of the first term on the RHS of Eq. (8.16) larger than the coefficient of the second term: $\left(\Delta \alpha_{e}^{\prime} / \Delta \alpha_{g}^{\prime}-R\right)>(R-1)$. At the early time, $t<500 \mathrm{ps}$, the birefringence is positive because the first term dominates. As the first term decays away with time constant of $\tau_{Q_{e}}=930 \mathrm{ps}$, the second term, with its negative magnitude and much longer decay time of $\tau_{g}=16.5 \mathrm{~ns}$, becomes increasingly important. Thus, as time passes the birefringence goes to zero, becomes negative, and, for $t \geq 3 \mathrm{~ns}$, flattens out at a negative value, as seen in Fig. (8.2).

Quantitatively, we can fit the time evolution of the induced birefringence with Eq. (8.16), where the fitting parameter is the ratio of the two coefficients of the two exponentials. We obtained a relatively satisfactory fit, shown in Fig. (8.2), from which we found: $\left(\Delta \alpha_{e}^{\prime} / \Delta \alpha_{g}^{\prime}-R\right) /(R-1)=-1.8$. (Note that since we have used the mean-field approximation in describing the rotational dynamics of the system, we don't expect to be able to describe accurately the early dynamics where $t \leq 500$ ps.)

From the measured values of $\tau_{Q_{e}}$ and $\tau_{g}$, we could deduce $\tau_{D_{e}}=980 \pm 100 \mathrm{ps}$ (knowing $\tau_{f}=18.7$ ns from Ref. [94]) and $\tau_{D_{g}}=820 \pm 40$ ps. Then for Eq. (8.8) we have $r_{\tau} \equiv \tau_{D_{g}} / \tau_{D_{e}}=0.84$ and $r_{f} \equiv \tau_{D_{g}} / \tau_{f}=0.044$. Thus if we know $\left(\Delta \alpha_{e}^{\prime} / \Delta \alpha_{g}^{\prime}\right)$, we can find $R$, then we can determine the ratio of the interaction energies $r_{u} \equiv u_{g g} / u_{g e}$.

To measure $\left(\Delta \alpha_{e}^{\prime} / \Delta \alpha_{g}^{\prime}\right)$ we would need to carry out two measurements to get the absolute values of (i)the birefringence created right after the excitation pulse is over and 
(ii)the excitation energy density. These information, combined with the known value of $\Delta \alpha_{g}^{\prime}$, the measured absorption coefficient of the pump beam, and some other experimental details, should allow us to calculate $\left(\Delta \alpha_{e}^{\prime} / \Delta \alpha_{g}^{\prime}\right)$. These measurements, though tedious, are straightforward, and we are currently working to carry them out.

The above measurements would also allow us to calculate the enhancement factor $\eta$, analogous to those in Eq. (3.21) and (3.25) of the dye-doped liquid crystal case, which measures how much extra molecular reorientation is gained as compared to the direct optical torque interaction. We actually could obtain an estimate of $\eta$ at the long time, $t \simeq 4 \mathrm{ns,}$ from preliminary measurements of (i) and (ii) above. The birefringence signal in Fig. (8.2) at $t=4$ ns was measured in absolute units yielding $S_{\text {observed }}$. Then, the corresponding signal that comes from the torque interaction alone, $S_{\text {torque }}$, was calculated from Eqs. (3.20) and (3.27), using the measured excitation energy density, the appropriate real polarizability anisotropies of $5 \mathrm{CB}$ at the pump and probe wavelengths [95], and the consideration that the pump intensity is attenuated as it traverses the absorbing sample (section 4.1). From the values of $S_{\text {observed }}$ and $S_{\text {torque }}$ we found that $\eta \equiv S_{\text {observed }} / S_{\text {torque }} \sim 20$. (Note that the heterodyned signal $S$ here is linear in $I$, while the non-heterodyned signal in Chapter 4 is quadratic in $I$.)

It turned out that the liquid crystal sample exhibited damage and altered optical properties (monitored by fluorescence) after repeated exposure to UV excitation. This limited the total number of laser shots that could be used to obtain the data, after which the sample had to be moved to allow exposure of a different spot on the sample cell, or the sample replaced altogether. This undoubtedly degraded the signal-to-noise ratio, and 
probably contributed to the considerable scattering in the data presented in Fig. (8.2). (Each data point shown is an average of about 35 laser shots, normalized shot-by-shot by the probe beam energy, and with the pump energy fluctuation kept within $\pm 20 \%$.) We are currently working on constructing a flow-cell to hold the liquid crystal sample, in order to reduce the damage problem. This is particularly important for accurate measurement of the absolute value of the induced birefringence discussed above.

While a full quantitative understanding is still missing, the results shown in this chapter have already established several important conclusions. Specifically, the result $\left(\Delta \alpha_{e}^{\prime} / \Delta \alpha_{g}^{\prime}-R\right) /(R-1)=-1.8$ clearly shows that $R<1$. This means that, first, the reorientational effect of the state-dependent intermolecular interaction is significant. Electronic excitation indeed could lead to enhanced optical reorientation in pure isotropic liquid crystals (enhancement factor $\sim 20$ observed in our case). Secondly, we have shown that the observed enhancement is negative, i.e. the net anisotropy created is perpendicular to the polarization direction of the pump field. Furthermore, from Eq. (8.8), and knowing experimentally that $r_{\tau}<1$ (see above), the fact that $R<1$ means we must have $r_{u} \equiv u_{g g} / u_{g e}>1$. The ground-state/ground-state orientational coupling is actually stronger than the groundstate/excited-state coupling.

All of the insights gained so far should be directly applicable to the case when the liquid crystal system is in the nematic phase. In fact, an interesting prospect for future work would be to look for the excitation-enhanced optical reorientation in pure nematic liquid crystals.

In summary, we have successfully observed the Jánossy effect in a pure liquid 
crystalline system. Though a full quantitative understanding is still in progress, much insights have been gained, thus significantly expanding our understanding of the problem. 


\section{Bibliography}

[1] I. C. Khoo and S. T. Wu, Optics and Nonlinear Optics of Liquid Crystals (World Scientific, New Jersey, 1993).

[2] B. Bahadur, in Handbook of Liquid Crystals, edited by D. Demus, J. Goodby, G. W. Gray, H.-W. Spiess, and V. Vill (Wiley-VCH, New York, 1998).

[3] H.-G. Kuball and T. Höfer, in Chirality in Liquid Crystals, edited by H.-S. Kitzerow and C. Bahr (Springer, New York, 2001).

[4] T. Ikeda, J. Materials Chem. 13, 2037 (2003).

[5] L. Marrucci, Liq. Cryst. Today 11, 1 (2002).

[6] L. Marrucci and Y. R. Shen, in The Optics of Thermotropic Liquid Crystals, edited by R. Sambles and S. Elston (Taylor \& Francis, London, 1998).

[7] I. Jánossy, A. D.Lloyd, and B. S. Wherrett, Molecular Crystals and Liquid Crystals 179, 1 (1990).

[8] I. Jánossy and A. D.Lloyd, Molecular Crystals and Liquid Crystals 203, 77 (1991). 
[9] I. Jánossy, L. Csillag, and A. D.Lloyd, Physical Review A 44, 8410 (1991).

[10] I. Jánossy and T. Kosa, Optics Letters 17, 1183 (1992).

[11] I. C. Khoo, H. Li, and Y. Liang, IEEE Journal of Quantum Electronics 29, 1444 (1993).

[12] D. Paparo, P. Maddalena, G. Abbate, E. Santamato, and I. Jánossy, Molecular Crystals and Liquid Crystals 251, 73 (1994).

[13] L. Marrucci, D. Papparo, P. Maddalena, E. Massera, E. Prudnikova, and E. Santamato, Journal of Chemical Physics 107, 9783 (1997).

[14] L. Marrucci, D. Paparo, M. R. Vetrano, M. Colichio, E. Santamato, and G. Viscardi, Journal of Chemical Physics 113, 10362 (2000).

[15] M. Kreuzer, F. Hanisch, R. Eidenschink, D. Paparo, and L. Marrucci, Physical Review Letters 88, 013902 (2002).

[16] K. Ichimura, Chemical Reviews 100, 1847 (2000).

[17] D. Paparo, L. Marrucci, G. Abbate, E. Santamato, M. Kreuzer, P. Lehnert, and T. Vogeler, Physical Review Letters 78, 38 (1997).

[18] D. Paparo, L. Marrucci, G. Abbate, E. Santamato, M. Kreuzer, P. Lehnert, and T. Vogeler, Physical Review A 58, 4926 (1998).

[19] R. Muenster, M. J. X. Zhuang, and Y. R. Shen, Physica Review Letters 78, 42 (1997).

[20] I. Jánossy, Physical Review E 49, 2957 (1994). 
[21] L. Marrucci and D. Paparo, Physical Review E 56, 1765 (1997).

[22] M. Kreuzer, L. Marrucci, and D. Paparo, Journal of Nonlinear Optical Physics and Materials 9, 157 (2000).

[23] I. Jánossy, Journal of Nonlinear Optical Physics and Materials 8, 361 (1999).

[24] P. Palffy-Muhoray, T. Kosa, and E. Weinan, Applied Physics A 75, 293 (2002).

[25] T. V. Truong, L. Xu, and Y. R. Shen, Physical Review Letters 90, 193902 (2003).

[26] T. V. Truong and Y. R. Shen, Journal of Chemical Physics 122, 091104 (2005).

[27] T. V. Truong, L. Xu, and Y. R. Shen, Physical Review E 72, 051709 (2005).

[28] R. M. Stratt and M. Maroncelli, Journal of Physical Chemistry 100, 12981 (1996).

[29] G. R. Fleming and M. Cho, Annual Review of Physical Chemistry 47, 109 (1996).

[30] E. W. Castner and M. Maroncelli, Journal of Molecular Liquids 77, 1 (1998).

[31] D. F. Underwood and D. A. Blank, Journal of Physical Chemistry 107, 956 (2003).

[32] S. J. Schmidtke, D. F. Underwood, and D. A. Blank, Journal of American Chemical Society 126, 8620 (2004).

[33] S. Park, B. N. Flanders, X. Shang, R. A. Westervelt, J. Kim, and N. F. Scherer, Journal of Chemical Physics 118, 3917 (2003).

[34] P. G. de Gennes, The Physics of Liquid Crystals (Claredon Pres, Oxford, 1974). 
[35] H. Inoue, T. Hoshi, J. Yoshino, and Y. Tanizaki, Bulletin of the Chemical Society of Japan 45, 1018 (1972).

[36] J. A. Delaire and K. Nakatani, Chemical Reviews 100, 1817 (2000).

[37] Y. R. Shen, The Principles of Nonlinear Optics (John Wiley \& Sons, New York, 1984).

[38] S. J. Clark, in Physical Properties of Liquid Crystals: Nematics, edited by D. A. Dunmur, A. Fukuda, and G. R. Luckhurst (INSPEC, London, 2000), pp. 489-497.

[39] J. N. Israelachvili, Intermolecular and Surface Forces (Academic Press, Inc., San Diego, 1987).

[40] P. G. de Gennes, Physics Letters A 30, 454 (1969).

[41] P. G. de Gennes, Molecular Crystals and Liquid Crystals 12, 193 (1971).

[42] Introduction to Liquid Crystals, edited by E. B. Priestley, P. J. Wojtowicz, and P. Sheng (Plenum Press, New York, 1975).

[43] I. T. W. Stinson and J. D. Lister, Physical Review Letters 25, 503 (1970).

[44] J. Prost and J. R. Lalanne, Physical Review A 8, 2090 (1973).

[45] G. K. L. Wong and Y. R. Shen, Physical Review Letters 30, 895 (1973).

[46] G. K. L. Wong and Y. R. Shen, Physical Review A 10, 1277 (1974).

[47] C. Flytzanis and Y. R. Shen, Physical Review Letters 33, 14 (1974).

[48] N. M. Amer, Y. S. Lin, and Y. R. Shen, Solid State Communications 16, 1157 (1975). 
[49] J. R. Lalanne, B. Martin, B. Pouligny, and S. Kielich, Optics Communications 19, 440 $(1976)$.

[50] J. R. Lalanne, B. Martin, B. Pouligny, and S. Kielich, Molecular Crystals and Liquid Crystals 43, 153 (1977).

[51] E. G. Hanson, Y. R. Shen, and G. K. L. Wong, Physical Review A 14, 1281 (1976).

[52] H. J. Coles, Molecular Crystals and Liquid Crystals (Lett.) 49, 67 (1978).

[53] B. Pouligny, E. Sein, and J. R. Lalanne, Physical Review A 21, 1528 (1980).

[54] F. W. Deeg, S. R. Greenfield, J. J. Stankus, V. J. Newell, and M. D. Fayer, Journal of Chemical Physics 93, 3503 (1990).

[55] A. Sengupta and M. D. Fayer, Journal of Chemical Physics 102, 4193 (1994).

[56] R. Torre, M. Ricci, G. Saielli, P. Bartolini, and R. Righini, Molecular Crystals and Liquid Crystals 262, 291 (1995).

[57] R. Torre and S. Califano, Journal de Chimie Physique 93, 1843 (1996).

[58] S. Ravichandran, A. Perera, M. Moreau, and B. Bagchi, Journal of Chemical Physics 109, $7349(1998)$.

[59] H. Cang, J. Li, V. N. Novikov, and M. D. Fayer, Journal of Chemical Physics 119, $10421(2003)$.

[60] P. P. Jose and B. Bagchi, Journal of Chemical Physics 120, 11256 (2004). 
[61] J. J. Stankus, R. Torre, and M. D. Fayer, Journal of Chemical Physics 97, 9478 (1993).

[62] R. Zwanzig, Journal of Chemical Physics 38, 2766 (1963).

[63] M. Kreuzer, E. Benkler, D. Paparo, G. Casillo, and L. Marrucci, Physical Review E 68, 011701 (2003).

[64] R. W. Boyd, Nonlinear Optics (Academic Press, Inc., San Diego, 1992).

[65] T. Kosa and I. Jánossy, Optics Letters 20, 1230 (1995).

[66] C. Manzo, D. Paparo, S. Lettieri, and L. Marrucci, Molecular Crystals and Liquid Crystals 421, 145 (2004).

[67] M. A. Duguay and J. W. Hansen, Applied Physics Letters 15, 192 (1969).

[68] G. R. Fleming, J. M. Morris, and G. W. Robinson, Chemical Physics 17, 91 (1976).

[69] D. S. Alavi, R. S. Hartman, and D. H. Waldeck, Journal of Chemical Physics 94, 4509 (1991).

[70] N. Pfeffer, F. Charra, and J. M. Nunzi, Optics Letters 16, 1987 (1991).

[71] G. S. Beddard and M. J. Westby, Chemical Physics 57, 121 (1981).

[72] I. Carmichael and G. L. Hug, Applied Spectroscopy 41, 1033 (1987).

[73] I. Carmichael, W. P. Helman, and G. L. Hug, Journal of Physical and Chemical Reference Data 16, 239 (1987).

[74] D. Paparo, C. Manzo, and L. Marrucci, Journal of Chemical Physics 117, 2187 (2002). 
[75] D. Reiser and A. Laubereau, Optics Communications 42, 329 (1982).

[76] G. J. Blanchard and C. A. Cihal, Journal of Physical Chemistry 92, 5950 (1988).

[77] G. J. Blanchard, Journal of Physical Chemistry 92, 6303 (1988).

[78] S. Alavi, R. S. Hartman, and D. H. Waldeck, Journal of Chemical Physics 95, 6770 (1991).

[79] A. N. Rubinov and B. A. Bushuk, Laser Physics 6, 514 (1996).

[80] I. Rückmann, A. Zeug, T. von Feilitzsch, and B. Röder, Optics Communications 170, 361 (1999).

[81] G. Eyring and M. D. Fayer, Journal of Chemical Physics 81, 4314 (1984).

[82] H. L. Eichler, P. Günter, and D. W. Pohl, Laser Induced Dynamic Grating (SpringerVerlag, Berlin, 1986).

[83] D. McMorrow, W. T. Lotshaw, and G. A. Kenney-Wallace, IEEE Journal of Quantum Electronics 24, 443 (1988).

[84] P. P. Ho and R. R. Alfano, Physical Review A 20, 2170 (1979).

[85] R. J. W. Fèvre, A. Sundaram, and K. M. S. Sundara, Journal of the Chemical Society 947 (1963).

[86] H. Inoue, M. Hida, N. Nakashima, and K. Yoshihara, Journal of Physical Chemistry 86, 3184 (1982). 
[87] M. A. Osipov and E. M. Terentjev, Zeitschrift für Naturforschung A 44, 785 (1989).

[88] A. Ferrarini, G. J. Morro, and P. L. Nordio, Physical Review E 53, 681 (1996).

[89] M. A. Osipov and H. G. Kuball, The European Physical Journal E 5, 589 (2001).

[90] G. Germano, M. P. Allen, and A. J. Masters, Journal of Chemical Physics 116, 9422 (2002).

[91] A. Y. Bobrovsky, N. I. Boiko, V. P. Shibaev, and J. Springer, Advanced Materials 12, $1180(2000)$.

[92] R. A. van Delden, M. B. van Gelder, N. P. M. Huck, and B. L. Feringa, Advanced Functional Materials 13, 319 (2003).

[93] H. G. Kuball and H. Bruning, Chirality 9, 407 (1997).

[94] T. Ikeda, S. Kurihara, and S. Tazuke, The Journal of Physical Chemistry 94, 6550 (1990).

[95] J. Lin and S.-T. Wu, Journal of Applied Physics 95, 896 (2004). 


\section{Appendix A}

\section{Mean-field description of rotational}

\section{dynamics}

\section{A.1 Introduction}

In this appendix we describe the derivation of the dynamical equations that govern the rotational dynamics of the guest-host system under the mean-field approximation. In chapter 3 the mean-field approximation was assumed for the guest-host interaction but not for the host-host interaction. The latter is particularly strong for liquid crystals, and has to be taken beyond the mean-field approximation (as was done in section 3.3) in order to describe adequately the rotational dynamics in the early time regime $(\leq 1 \mathrm{~ns})$ following a picosecond-pulsed excitation. However, at longer time or in the stead-state regime, the faster relaxation channels of the liquid crystals that come from perturbation to the meanfield, the $k$-terms with $k>1$ in Eq. 3.16, have decayed away, and only the $k=1$ term, 
which is described by the mean-field interaction, remains important.

Thus it is instructive to derive the dynamical equations for the guest-host system where both the guest-host and host-host interactions are described by a mean-field. Under this approximation, the dynamical equations for both the guest and host population can be derived through the same unified formalism which is used in section 3.2.1 to derive the equations for the dye population. The derivation will help us gain a clearer physical picture. We will find that for the host we arrive at an equation of motion similar to Eq. (3.21) but only with the $k=1$ term. Our derivation will allow us to:

(i) understand why the intermolecular interaction has to be taken into account when considering the reorientational effect of the guest on the host, but can be neglected when considering the effect of the host on the guest.

(ii) derive the dynamical equations for the case when the system is consisted of pure liquid crystal and the optical field excites the liquid crystal molecules themselves (Ch. $8)$.

\section{A.2 Dynamics of dye-doped liquid crystals}

The first steps are the same as in Chapter 3. We rewrite them here for easier referencing. We begin with the definitions of orientational distribution functions $f_{i}$ and the associated Legendre moments $Q_{i}^{(l)}$ (Eqs. 3.1-2):

$$
f_{i}(\theta, t)=\frac{1}{4 \pi} \sum_{l=0,2,4, \ldots}(2 l+1) Q_{i}^{(l)}(t) P_{l}(\cos \theta)
$$


where

$$
Q_{i}^{(l)}(t)=\int f_{i}(\theta, t) P_{l}(\cos \theta) d \Omega
$$

with $i=h, e, g$ representing the host, excited-state dye, and ground-state dye molecular species, respectively. All odd-l moments are zero due to the inversion symmetry of the system, the zeroth-order moment $Q_{i}^{(0)}(t)=N_{i}(t)$ is the number density of the $i$-type molecular species, and the second-order moment divided by the number density, $Q_{I}^{(2)}(t) / N_{i}(t)$, is the usual orientational order parameter.

In the diffusional limit, the distribution functions obey the Einstein-Schomulchowski equation:

$$
\frac{\partial}{\partial t} f_{i}-D_{i} \frac{1}{\sin \theta} \frac{\partial}{\partial \theta} \sin \theta\left(\frac{\partial}{\partial \theta}+\frac{1}{k_{B} T} \frac{\partial U_{i}}{\partial \theta}\right) f_{i}=W_{i}
$$

with $D_{i}$ denoting the rotational diffusion coefficient and $k_{B} T$ the thermal energy. Here $W_{i}$ is the rate of population transition affecting the $i$-type molecules. Since the optical excitation is in resonance with the dye only, we have $W_{h}=0$ and

$$
W_{e}(\theta, t)=-W_{g}(\theta, t)=\left(\frac{3 \beta I \cos ^{2} \theta}{h \nu N_{d}}\right) f_{g}(\theta, t)-\frac{f_{e}(\theta, t)}{\tau_{f}},
$$

where $\nu$ and $I$ are the optical pump frequency and intensity, respectively, $\beta$ is the linear absorption coefficient, $h$ is the Planck's constant, $N_{d}$ is the total dye number density, and $\tau_{f}$ is the excited-state lifetime.

The orientational potential energy $U_{i}(\theta)$ is consisted of two contribution:

$$
U_{i}(\theta)=U_{i}^{E M}+U_{i}^{i n t} .
$$

representing, respectively, the direct torque exerted by the external electromagnetic field 
and the reorientational effect of intermolecular interactions. The first term is given by:

$$
U_{i}^{E M}=-\frac{2 \pi L^{2} \Delta \alpha_{i}^{\prime}}{n c} I \cos ^{2} \theta
$$

where $L$ is the local field factor, $\Delta \alpha_{i}^{\prime}$ is the real polarizability anisotropy of an $i$-type molecule, $n$ is the refractive index, and $c$ is the vacuum light speed. The second term could be expressed as the sum of microscopic pair-wise interaction (the Maier and Saupe potential $[42]):$

$$
U_{i}^{i n t}=-\sum_{j} \sum_{n} G_{i j}^{m n} s_{i}^{m} s_{j}^{n} \quad n \neq m, j \neq i,
$$

where $s_{i}^{m} \equiv(3 / 2) \cos ^{2} \theta_{i}^{m}-(1 / 2)$ describes the orientation of the $m$-th molecule of the $i$-type species (Eq. (3.3)). In the mean-field approximation, the interaction potential can be written as:

$$
U_{i}^{i n t}=-\frac{1}{2} \sum_{j} u_{i j} \frac{Q_{j}^{(2)}}{N_{t}} \cos ^{2} \theta \quad \text { with } \quad u_{i j} \equiv \frac{3 N_{t}}{N_{j}}\left\langle\sum_{n} G_{i j}^{m n}\right\rangle .
$$

We now discuss the physical meaning of the expressions in Eqs. (A.7-8). The symmetric energy coefficients $u_{i j}$ gauges the average strength of the orientational coupling between one pair of $i$ and $j$-type molecules. We see that $u_{i j}$ is obtained by summing up the individual pair-wise coupling coefficients $G_{i j}^{m n}$, take the ensemble average, and then normalized by the number of molecules involved. The ensemble average here means taking the average as the $m$-index run over all of the molecules of the $i$-type population. The dependence of the orientational coupling on the intermolecular distance is embedded in $G_{i, j}^{m, n}$. It is known that intermolecular interaction strength drops off sharply as a function of distance $\left(\propto r^{-3}\right.$ for dipole-dipole interaction, see [39]), so the sum that makes up $u_{i j}$ in 
Eq. (A.7) is dominated by just a few terms that account for the nearest-neighbor pairs. The LC-LC and LC-dye distances are expected to be about the same, while the dye-dye distance is $\geq 10^{3}$ times the LC-LC distance, due to the low dye concentration. Thus we expect (i) $u_{h h}, u_{h e}$ and $u_{h g}$ are of the same order of magnitude (with the difference coming from specific molecular differences between the LC and dye molecules); (ii) $u_{i j}$ for the case $i, j=e, g$ will be much smaller than for the case when either $i$ or $j=h$.

With all the orientational potentials of Eq. (A.3) described, we next use the definition of the Legendre moments, Eq. (A.2), to convert the diffusion equation, Eq. (A.3), into an equivalent set of ordinary differential equations for the moments $Q_{i}^{(l)}(t)$. We get $[18]$ :

$$
\begin{aligned}
& \frac{\partial Q_{i}^{(l)}}{\partial t}+l(l+1) D_{i}\left\{Q_{i}^{(l)}+\left[\frac{4 \pi L^{2} \Delta \alpha_{i}^{\prime} I}{k_{B} T n c}+\sum_{j} u_{i j} \frac{Q_{j}^{(2)}}{k_{B} T N_{t}}\right] \cdot\left[-\frac{l-1}{(2 l-1)(2 l+1)} Q_{i}^{(l-2)}\right.\right. \\
& \left.\left.-\frac{1}{(2 l-1)(2 l+3)} Q_{i}^{(l)}+\frac{l+2}{(2 l+1)(2 l+3)} Q_{i}^{(l+2)}\right]\right\}=R_{i}^{(l)},
\end{aligned}
$$

with $R_{h}^{(l)}=0$ and $R_{e}^{(l)}=-R_{g}^{(l)}=$

$$
\begin{aligned}
=-\frac{Q_{e}^{(l)}}{\tau_{f}}+\frac{3 \beta I}{h \nu N_{d}}\left[\frac{l(l-1)}{(2 l-1)(2 l+1)} Q_{g}^{(l-2)}\right. & +\frac{2 l^{2}(2 l+3)-1}{(2 l-1)(2 l+1)(2 l+3)} Q_{g}^{(l)} \\
& \left.+\frac{(l+1)(l+2)}{(2 l+1)(2 l+3)} Q_{g}^{(l+2)}\right]
\end{aligned}
$$

The above constitute the full set of equations of motion for all nonzero Legendre moments of the distribution function. We see that in general, the $l$-moment is coupled to the $(l-2)$ and $(l+2)$ moments. For our study, we employ relative weak optical pumping intensity. This means that the induced orientational ordering is small with $Q_{i}^{(4)} \ll Q_{i}^{(2)}$, and thus to describe the orientational ordering, the second moment is adequate. From now on we will 
focus only on the second moment, and simply write it as $Q_{i}$. The weak excitation intensity also leads to a small population inversion for the dye $\left(\leq 10^{-2}\right)$, hence $N_{e}, Q_{e}, Q_{g} \ll N_{d} \simeq$ $N_{g}$

With the above approximations applied to Eqs. (A.9) and (A.10), the dynamical equations for the anisotropies $Q_{i}$ simplify significantly. After rearranging the terms, we can write these equations in the following form:

$$
\frac{\partial Q_{i}}{\partial t}+\frac{Q_{i}}{\tau_{D_{i}}}=R_{i}+T_{i}+X_{i}
$$

where the terms on the right hand side represent the various factors that affect the orientational anisotropy of the $i$-type species: the photoinduced population transition, the photoinduced torque reorientation, and the mean-field reorientational intermolecular interaction, respectively. These terms, and their relative importance to the equations of motion, will be discussed below.

For the population transition, we have $R_{h}=0$ and:

$$
R_{e}=-R_{g}=-\frac{Q_{e}}{\tau_{f}}+A I(t), \text { with } A \equiv \frac{2 \beta}{5 h \nu} .
$$

Numerically, with $\beta=6.8 \mathrm{~cm}^{-1}, h \nu=2.33 \mathrm{eV}$, and $I \simeq 10^{7} \mathrm{~J}^{-1} \mathrm{~cm}^{-2} \mathrm{~s}^{-1}$ (instananeously), we have $A I \simeq\left(7.3 \times 10^{18} \mathrm{~J}^{-1} \mathrm{~cm}^{-1}\right) I=7.3 \times 10^{25} \mathrm{~cm}^{-3} \mathrm{~s}^{-1}$.

The torque interaction term is given by:

$$
T_{i}=\frac{4 \pi L^{2} \Delta \alpha_{i} N_{i}}{15 k_{B} T n c \tau_{D_{i}}} I(t)
$$

with $\tau_{D_{i}} \equiv 1 / 6 D_{i}$ is the rotational diffusion time constant of the $i$-type species. Numerically, $T_{h} \simeq\left(3.8 \times 10^{17} \mathrm{~J}^{-1} \mathrm{~cm}^{-1}\right) I=3.8 \times 10^{24} \mathrm{~cm}^{-3} \mathrm{~s}^{-1}$. For the dye molecules, we expect $\Delta \alpha_{e, g}^{\prime} \leq \Delta \alpha_{h}$ and $\tau_{D_{e, g}} \simeq \tau_{D_{h}}$, then $T_{e} \simeq T_{g} \leq\left(N_{e, g} / N_{h}\right) T_{h} \leq 1 \times 10^{-3} T_{h}$. 
Comparing the magnitude of the $R_{i}$ and $T_{i}$ terms, we see that in general the torque interaction is much smaller and can be neglected when there is resonance absorption (even with the case of relatively moderate absorption considered here). Thus the $T_{e, g}$ terms can be neglected in the equations for $Q_{e, g}$.

The intermolecular interaction terms of Eq. (A.11) are given by:

$$
\begin{aligned}
X_{h} & =\frac{u_{h h}}{15 k_{B} T \tau_{D_{h}}} \frac{N_{h} N_{h}}{N_{t}} \frac{Q_{h}}{N_{h}}+\frac{u_{h e}}{15 k_{B} T \tau_{D_{h}}} \frac{N_{h} N_{e}}{N_{t}} \frac{Q_{e}}{N_{e}}+\frac{u_{h g}}{15 k_{B} T \tau_{D_{h}}} \frac{N_{h} N_{g}}{N_{t}} \frac{Q_{g}}{N_{g}} \\
X_{e} & =\frac{u_{e e}}{15 k_{B} T \tau_{D_{e}}} \frac{N_{e} N_{e}}{N_{t}} \frac{Q_{e}}{N_{e}}+\frac{u_{e h}}{15 k_{B} T \tau_{D_{e}}} \frac{N_{e} N_{h}}{N_{t}} \frac{Q_{h}}{N_{h}}+\frac{u_{e g}}{15 k_{B} T \tau_{D_{e}}} \frac{N_{e} N_{g}}{N_{t}} \frac{Q_{g}}{N_{g}} \\
X_{g} & =\frac{u_{g g}}{15 k_{B} T \tau_{D_{g}}} \frac{N_{g} N_{g}}{N_{t}} \frac{Q_{g}}{N_{g}}+\frac{u_{g h}}{15 k_{B} T \tau_{D_{g}}} \frac{N_{g} N_{h}}{N_{t}} \frac{Q_{h}}{N_{h}}+\frac{u_{g e}}{15 k_{B} T \tau_{D_{g}}} \frac{N_{g} N_{e}}{N_{t}} \frac{Q_{e}}{N_{e}} .
\end{aligned}
$$

We see that each term on the right hand side (RHS) can be written in the form:

$$
\frac{u_{i j}}{15 k_{B} T \tau_{D_{i}}} \frac{N_{i} N_{j}}{N_{t}} \frac{Q_{j}}{N_{j}}
$$

describing the mean-field reorientational effect that the $j$ population has on the $i$ population.

The physical meanings of the parameters in Eq. (A.17) are as follow. The symmetric energy coefficients $u_{i j}$ gauges the average strength of interaction between a pair of $i$ and $j$ molecules. The number densities $N_{i}$ and $N_{j}$ in the numerator gauge how many molecules each species has. The quantity $Q_{j} / N_{j}$ is the dimensionless order parameter of the $j$ species. We expect $\tau_{D_{i}}$ to be of roughly the same order of magnitude for all species. Then, from the conditions of low dye-doping concentration and low optical pumping intensity, we can compare the magnitudes of the various terms on the RHS of Eqs. (A.14-16) to that of the term proportional to $u_{h h}$ in Eq. (A.14). The latter is used as the reference since we know that its effect is significant enough to produce the pre-transitional behavior in pure liquid crystal rotational dynamics as described before. 
We first look at the three terms affecting the host population on the RHS of Eq. (A.14). We know already that $u_{h h}, u_{h e}$ and $u_{h g}$ should be of the same order of magnitude. From the values of $R_{e}$ and $T_{h}$ estimated before, we could estimate that, immediately after the picosecond excitation pulse, $Q_{e} / Q_{h} \simeq 20$ (this ratio is reduced to $\simeq 2.5$ if we include the contribution to $Q_{h}$ from the faster responses described in section 3.2.3). Thus the contributions of the latter two terms on the RHS of Eq. (A.14), representing the effects of the dye anisotropies on the host population, are quite significant. This guest-induced reorienting of the host is the essence of the Jánossy effect.

Also, knowing that $u_{h i} \sim 0.1 \mathrm{eV}$ for typical nearest-neighbor interaction in the liquid state [39], it readily follows that the integrated effect of $X_{h}$ on $Q_{h}\left(\simeq X_{h} \tau_{D_{h}}\right)$ is of the same order or larger compared to the integrated effect of the direct torque term $\left(\simeq T_{h} \times\right.$ laser pulse width). This is in effect the same estimate that we made in section 3.1 to demonstrate that guest-host interaction is large enough to compete with, and surpass, the direct electromagnetic torque in creating host anisotropy.

Next we look at the terms affecting the dye population, Eqs. (A.15-16). We have already established that $u_{i j}$ with $i, j=e, g$ is much smaller than those with either $i$ or $j=h$ due to the low dye-doping concentration. Thus all terms involving dye-dye interaction affecting $X_{e}$ and $X_{g}$ in Eq. (A.15-16) can be neglected. For the terms that involve $u_{e h}$ and $u_{g h}$ in Eqs. (A.15-16), we see that they are negligible compared to the terms that involve $u_{h e}$ and $u_{h g}$ in Eq. (A.14) because $Q_{h} / N_{h}\left(\leq 10^{-5}\right)$ is much smaller than $Q_{e} / N_{e}(\simeq 2 / 5)$ or $Q_{g} / N_{g}\left(\leq 10^{-2}\right)$ with the low excitation intensity used in our study. So overall, we see that the reorientational effects that the environment has on the dye molecules are comparatively 
small, and thus $X_{e}$ and $X_{g}$ could be neglected in the equations of motion for $Q_{e}$ and $Q_{g}$.

We summarize our discussion of the terms on the RHS of Eq. (A.11) that affect the orientational dynamics of the guest-host system. For the dye population, only the polarized population excitation is important and the effects from intermolecular interaction and the direct electromagnetic torque could be neglected. For the host population, no population excitation is involved and effects from both the intermolecular interaction and the direct torque have to be taken into account. We finally arrive at the following equations of motion for each of the molecular species:

$$
\begin{aligned}
\frac{\partial Q_{e}}{\partial t}+\frac{Q_{e}}{\tau_{D_{e}}} & =-\frac{Q_{e}}{\tau_{f}}+A I(t) \\
\frac{\partial Q_{g}}{\partial t}+\frac{Q_{g}}{\tau_{D_{g}}} & =+\frac{Q_{e}}{\tau_{f}}-A I(t) \\
\frac{\partial Q_{h}}{\partial t}+\frac{Q_{h}}{\tau_{D_{h}}}= & \frac{4 \pi L^{2} \Delta \alpha_{h}^{\prime} N_{h}}{15 k_{B} T \tau_{D_{h}} n c} I(t)+\frac{u_{h h} N_{h}}{15 k_{B} T \tau_{D_{h}} N_{t}} Q_{h} \\
& +\frac{u_{h e} N_{h}}{15 k_{B} T \tau_{D_{h}} N_{t}} Q_{e}+\frac{u_{h g} N_{h}}{15 k_{B} T \tau_{D_{h}} N_{t}} Q_{g} .
\end{aligned}
$$

Eqs. (A.18) and (A.19), describing the dye rotational dynamics, are the same as those in Eqs. (3.8). Rearranging the terms of Eq. (A.20) yields:

$$
\frac{\partial Q_{h}}{\partial t}+\left(1-\frac{u_{h h} N_{h}}{15 k_{B} T N_{t}}\right) \frac{Q_{h}}{\tau_{D_{h}}}=\frac{N_{h}}{15 k_{B} T \tau_{D_{h}} N_{t}}\left[B I(t)+u_{h e} Q_{e}(t)+u_{h g} Q_{g}(t)\right]
$$

which then can be written as:

$$
\frac{\partial Q_{h}}{\partial t}+\frac{Q_{h}}{\tau_{h}}=\frac{C_{h}}{\tau_{h}}\left[B I(t)+u_{h e} Q_{e}(t)+u_{h g} Q_{g}(t)\right]
$$

where

$$
\tau_{h} \equiv \tau_{D_{h}}\left(\frac{T}{T-T^{*}}\right), C_{h} \equiv \frac{N_{h}}{15 k_{B} N_{t}}\left(\frac{1}{T-T^{*}}\right), \text { with } T^{*} \equiv \frac{u_{h h} N_{h}}{15 k_{B} N_{t}}
$$


The solution to Eq. (A.22) is Eq. (3.20) in section 3.2 .3 with only the $k=1$ term, and identifying $\tau_{1}=\tau_{h}, C_{1}=C_{h}$. (Note that $N_{h} / N_{t} \simeq 1$.)

\section{A.3 Dynamics of pure liquid crystals}

The system now is consisted of the pure liquid crystal only, and light absorption takes place with the liquid crystal molecules themselves. In essence, light absorption in-

troduces to the system the "guest" population: the oriented excited molecules and the orientational holes in the ground state distribution. These will then act to reorient the surrounding molecules.

Thus in this case, there is no host, only $i=g$ and $e$ molecules. The dynamical equations can be readily derived using the same formalism used in the previous section for the dye-doped liquid crystal, keeping in mind that the $g$-type population now is equivalent to both the $g$ and $h$-type populations in the dye-doped case before. The dynamical equations analogous to Eq. (A.18-20) are:

$$
\begin{aligned}
\frac{\partial Q_{e}}{\partial t}+\frac{Q_{e}}{\tau_{D_{e}}} & =-\frac{Q_{e}}{\tau_{f}}+A I(t) \\
\frac{\partial Q_{g}}{\partial t}+\frac{Q_{g}}{\tau_{D_{g}}} & =+\frac{Q_{e}}{\tau_{f}}-A I(t)+\frac{u_{g g} N_{g}}{15 k_{B} T \tau_{D_{g}} N_{t}} Q_{g}+\frac{u_{g e} N_{g}}{15 k_{B} T \tau_{D_{g}} N_{t}} Q_{e} .
\end{aligned}
$$

Here we have neglected the effect of the direct torque interaction on the liquid crystal since, as we have seen earlier, the effect of resonant population transfer, the $A I(t)$ term, easily dominates the nonresonant torque interaction in creating population anisotropy. Equations (A.24-25) can then be used to describe the time evolution of the orientational dynamics of the pure absorbing liquid crystals, as was done in Chapter 8 . 